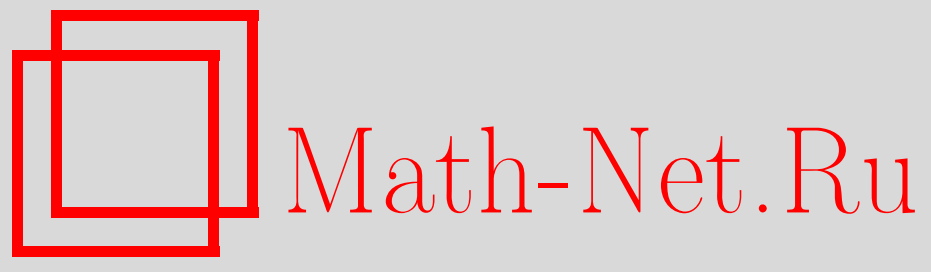

И. Д. Шкредов, Анализ Фурье в комбинаторной теории чисел, УМН, 2010, том 65, выпуск 3, 127-184

DOI: https://doi.org/10.4213/rm9355

Использование Общероссийского математического портала Math-Net.Ru подразумевает, что вы прочитали и согласны с пользовательским соглашением http://www . mathnet.ru/rus/agreement

Параметры загрузки:

IP : 54.209 .52 .79

26 апреля 2023 г., 10:49:07 


\section{Анализ Фурье в комбинаторной теории чисел}

\section{И. Д. Шкредов}

В обзоре рассматриваются приложения гармонического анализа к комбинаторной теории чисел. Обсуждаются классические проблемы аддитивной комбинаторики, задачи о раскрасках, анализ Фурье высших порядков, теоремы о множествах больших тригонометрических сумм, результаты об оценках тригонометрических сумм по подгруппам, связь между комбинаторной и аналитической теорией чисел.

Библиография: 162 названия.

Ключевые слова: анализ Фурье, комбинаторная теория чисел, аддитивная комбинаторика.

\section{СОДЕРЖАНИЕ}

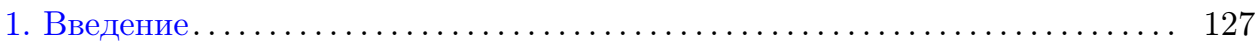

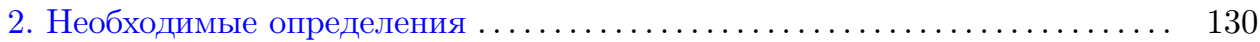

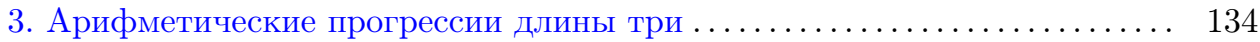

4. Анализ Фурье высших порядков . . . . . . . . . . . . . . . . . . . . . . . 142

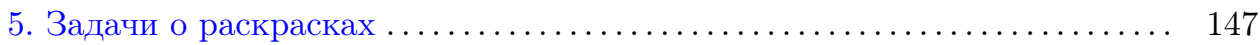

6. Множества больших тригонометрических сумм.................. 154

7. Комбинаторные конфигурации в суммах множеств .............. 161

8. Теорема Чеботарёва и принцип неопределенности .................. 168

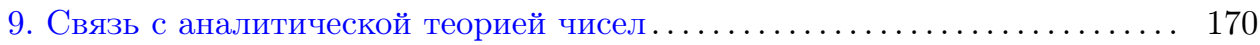

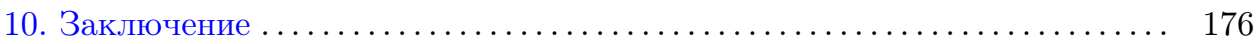

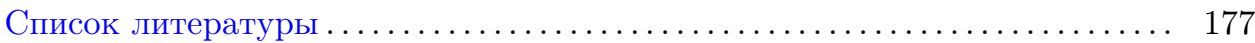

\section{1. Введение}

В настоящем обзоре мы рассмотрим приложения анализа Фурье к задачам комбинаторной теории чисел. Комбинаторная теория чисел или, как ее сейчас называют, аддитивная комбинаторика - это раздел математики, в котором

Работа выполнена при поддержке РФФИ (грант № 06-01-00383), гранта им. П. Делиня (фонд Бальзана 2004), гранта Президента РФ МK-1959.2009.1 и программы "Ведущие научные школы" (грант НШ-691.2008.1).

(C) И. Д. Шкредов, 2010 
изучают комбинаторные вопросы, связанные с групповой операцией (см. книги [1], [2] и обзоры [3], [4]). Хотя рассматриваемая область достаточно старая - достаточно вспомнить результат А. Л. Коши 1813 г. о сложении множеств в группе вычетов $\mathbb{Z} / p \mathbb{Z}$ (см. [5]), а также знаменитую теорему Л. Г. Шнирельмана 1933 г. (см. [6]) о представимости любого натурального числа в виде суммы ограниченного количества простых, но именно в последнее время интерес к рассматриваемой тематике значительно возрос, что связано, прежде всего, со значительным успехом в комбинаторных задачах об арифметических прогрессиях [7]-[9], появлением новых оценок для сумм произведений множеств в конечных полях [10]-[23], прогрессом в задачах комбинаторной эргодической теории [24]-[35], существенным продвижением в оценках тригонометрических сумм по подгруппам [14]-[23].

Не последнюю роль здесь сыграл метод анализа Фурье. Более того, самые сильные результаты в рассматриваемой области были получены именно с помощью гармонического анализа или, по крайней мере, были стимулированы вопросами анализа Фурье.

Выдающийся английский математик К.Ф. Рот первым применил гармонический анализ к задачам комбинаторной теории чисел. В 1953 г. он доказал ставший классическим результат о плотности подмножеств отрезка натурального ряда, не содержащих арифметических прогрессий длины три. Итерационный подход, который Рот использовал в своем доказательстве, нашел широчайшее применение в комбинаторной теории чисел и был переосмыслен с точки зрения теории графов (см. [36], [37]) и эргодической теории (см., например, [38], [39]). Наилучшие результаты по задаче Рота в настоящий момент принадлежат Ж. Бургейну (см. [40], [41]). Созданные им инструменты (прежде всего метод множеств Бора и результаты по большим тригонометрическим суммам) также систематически используются в целом ряде задач аддитивной комбинаторики.

Огромный вклад в развитие анализа Фурье в плане его приложений к задачам комбинаторной теории чисел сделал В. Т. Гауэрс (см. [7], [8]). Решая задачу о плотности множества без арифметических прогрессий длины больше, чем три, и обобщая метод Рота, Гауэрс создал так называемый “анализ Фурье высших порядков". Как известно, классический анализ Фурье сопоставляет произвольной функции набор ее скалярных произведений с групповыми характерами (коэффициенты Фурье). Оказывается, что в задачах об арифметических прогрессиях длиной больше, чем три, нужно рассматривать скалярные произведения функции и характера, аргумент которого есть некоторый многочлен. Получающиеся при этом аналоги коэффициентов Фурье играют чрезвычайно важную роль во всей этой проблематике (более подробно см. раздел 4). Методы Гауэрса развивались различными авторами (см., например, [9], [14], [15] [42]-[46]). Самым ярким из полученных в этих статьях результатов является, несомненно, теорема Б. Грина и Т. Тао, утверждающая, что простые числа содержат арифметические прогрессии сколь угодно большой длины.

Наконец, недавно обнаружилась связь между некоторыми задачами аддитивной комбинаторики и проблемами аналитической теории чисел. Так, в статье [15] с помощью оценок сумм произведений в группе $\mathbb{Z} / p \mathbb{Z}$ доказана равно- 
мерная распределенность любой мультипликативной подгруппы $R \subseteq(\mathbb{Z} / p \mathbb{Z})^{*}$, если размер $R$ достаточно большой, а именно $|R| \geqslant p^{\varepsilon}, \varepsilon>0$ - любое число. Это значительно усиливает аналогичные классические результаты (см., например, книгу [47]), где равномерная распределенность была известна лишь для подгрупп размера $p^{c}, c>1 / 4$ (см. [48]). Метод сумм произведений позволил получить нетривиальные оценки тригонометрических сумм и в других задачах (см. [49], [50]). С другой стороны, как было недавно отмечено в работах [51] и [52], наоборот, классические оценки тригонометрических сумм оказываются полезными в проблематике, связанной с суммами произведений. Приложения методов аналитической теории чисел к комбинаторным задачам рассматривались также в статьях [53]-[56]. Автор надеется, что обе указанные области еще окажут друг на друга взаимное влияние.

В настоящем обзоре мы будем рассматривать, в основном, задачи в конечных абелевых группах. Ясно, что если какая-то проблема аддитивной комбинаторики поставлена в некоторой конкретной группе, то ее можно переформулировать и в любой другой. При этом в одних группах $\mathbf{G}$ ответить на поставленный вопрос легко, а в других - трудно, поскольку, как выразился Б. Грин в [3], "не все группы созданы одинаковыми". Недавно выяснилось (см. [40], [57], [58] и, особенно, замечательный обзор [3]), что чрезвычайно удобно брать в качестве группы $\mathbf{G}$ группы $(\mathbb{Z} / p \mathbb{Z})^{n}$, где $p$ - маленькое простое число (например, $p=2,3$ или 5). Дело в том, что в таких группах имеется естественная структура векторного пространства и при этом сами группы $(\mathbb{Z} / p \mathbb{Z})^{n}$ не слишком сложно устроены. Более того, общая идеология статьи [3] состоит в следующем: если некоторый результат комбинаторной теории чисел доказан для групп $(\mathbb{Z} / p \mathbb{Z})^{n}$, то, как правило, соответствующий результат может быть доказан и для произвольной конечной абелевой группы, причем без привлечения принципиально новых идей. Все это говорит о том, что достаточно разумно иметь дело не с одной конкретной абелевой группой (скажем, $\mathbb{Z} / N \mathbb{Z}$ ), а обсудить всю имеющуюся совокупность абелевых групп.

Опишем вкратце структуру настоящего обзора. В разделе 2 мы напоминаем некоторые понятия анализа Фурье и приводим необходимые определения. Следующий раздел 3 посвящен доказательству теоремы Рота об арифметических прогрессиях длины три. Мы подробно обсуждаем случай группы $(\mathbb{Z} / 3 \mathbb{Z})^{n}$, а также получаем классическую теорему Рота. В разделе 4 мы затрагиваем молодую и бурно развивающуюся область гармонического анализа, так называемый “анализ Фурье высших порядков". Здесь имеется много открытых проблем и нерешенных вопросов, ждущих своих исследователей.

Традиционно при решении линейных уравнений анализ Фурье имел дело лишь с "плотностными" задачами (см. определения в разделе 5), такими как теорема Рота. Недавно молодой математик Т. Сандерс придумал метод, как использовать гармонический анализ в задачах о раскрасках - области, традиционно относящейся к чистой комбинаторике. Мы рассматриваем несколько имеющихся здесь проблем и результатов, таких как теорема Шура [59] и ее обобщения, в разделе 5. Тематика, связанная с изучением множеств больших тригонометрических сумм, обсуждаемая нами в разделе 6 , также является молодой и развивающейся областью. Достаточно сказать, что первый общий ре- 
зультат здесь был получен лишь в 2002 г. Тем не менее, любой прогресс в нашем понимании строения множеств больших тригонометрических сумм позволит, по мнению автора, продвинуться в большинстве тех задач аддитивной комбинаторики, где используется анализ Фурье. Эту же мысль высказывал Т. Гауэрс в обзоре [60]. В любом случае примеры такого продвижения можно видеть в работах [41], [61]-[66]. Далее (раздел 7) мы рассмотрим новую интересную область комбинаторной теории чисел, связанную с поиском различных конфигураций, прежде всего, арифметических прогрессий в суммах нескольких множеств. Первый результат в этом направлении принадлежит Ж. Бургейну [67]. Интересно отметить, что его метод доказательства не вписывается в рамки итерационного подхода, предложенного Ротом. В следующем разделе 8 затрагивается другой аспект гармонического анализа, а именно невырожденность (в широком смысле) преобразования Фурье. Мы обсудим изящную работу Т. Тао, посвященную так называемому “принципу неопределенности" в группе $\mathbb{Z} / p \mathbb{Z}, p$ - простое число. Оказывается, этот результат есть простая переформулировка не менее изящной теоремы нашего выдающегося математика Н.Г. Чеботарёва об определителях миноров матрицы преобразования Фурье. В разделе 9 рассматриваются связи между аддитивной комбинаторикой и аналитической теорией чисел. Обсуждаются задачи об оценках сумм произведений и их обобщений, а также несколько комбинаторных вопросов, в решении которых оказываются полезными чисто аналитические методы. Наконец, в заключении рассматривается ряд открытых проблем комбинаторной теории чисел, для решения которых анализ Фурье может иметь решающее значение.

K сожалению, выбор сюжетов для настоящего обзора определялся исключительно интересами автора. При этом, конечно, многие разделы аддитивной комбинаторики, использующие анализ Фурье, остались незатронутыми, например, огромная область, связанная с проблемой Какея. Автор может лишь предложить читателю ознакомиться с этой тематикой по замечательным обзорам [68], [69], а также работе [70].

В статье мы ограничимся, в основном, довольно старой областью классического гармонического анализа в свете его приложений к комбинаторной теории чисел. Анализу Фурье высших порядков, развитому в работах [7]-[9], [42], [71]-[76] и др., посвящен лишь один раздел 4. Тем не менее, мы надеемся, что прочитав наш обзор, читатель сможет убедиться, что и в этой части математики в последнее время произошло много нового.

\section{2. Необходимые определения}

В этом разделе мы напомним некоторые понятия анализа Фурье. Пусть $\mathbf{G}=(\mathbf{G},+)$ - локально компактная абелева группа с аддитивной групповой операцией + . Хорошо известно, что на всякой такой группе существует мера Хаара $\mu_{\mathbf{G}}$, т. е. неотрицательная регулярная мера (все определения могут быть найдены, например, в [77; гл. 1, 2 и добавление $E])$ такая, что для всякого борелевского множества $B$ и произвольного элемента $x \in \mathbf{G}$ имеем

$$
\mu_{\mathbf{G}}(B+x)=\mu_{\mathbf{G}}(B) .
$$


На пространстве $L_{2}\left(\mathbf{G}, \mu_{\mathbf{G}}\right)=L_{2}(\mathbf{G})$ задано скалярное произведение двух комплексных функций $f$ и $g$ :

$$
\langle f, g\rangle=\int_{\mathbf{G}} f(x) \overline{g(x)} d \mu_{\mathbf{G}}(x) .
$$

Пусть далее $\widehat{\mathbf{G}}$ - двойственная группа для группы $\mathbf{G}$, т. е. локально компактная абелева группа непрерывных гомоморфизмов $\xi$ из $\mathbf{G}$ в $\mathbf{S}^{1}=\{z \in \mathbb{C}:|z|=1\}$, $\xi: x \rightarrow \xi \cdot x=\xi(x), x \in \mathbf{G}$. Преобразование Фурье $(\Phi f)(\xi)=\hat{f}(\xi)$ произвольной комплексной функции $f \in L_{1}(\mathbf{G})$ задается формулой

$$
(\Phi f)(\xi)=\hat{f}(\xi)=\int_{\mathbf{G}} f(x) \overline{\xi(x)} d \mu_{\mathbf{G}}(x) .
$$

Ясно, что всегда справедливо неравенство

$$
\|\hat{f}\|_{\infty} \leqslant \int_{\mathbf{G}}|f(x)| d \mu_{\mathbf{G}}(x)=\|f\|_{1} .
$$

Пусть $\Gamma \subseteq \widehat{\mathbf{G}}-$ некоторое множество характеров и $\varepsilon \in[0,1]-$ действительное число. Определим множество Бора, порожденное множеством Г:

$$
\mathscr{B}(\Gamma, \varepsilon)=\left\{x \in \mathbf{G}:\|\xi \cdot x\| \leqslant \frac{\varepsilon}{2 \pi} \text { для всех } \xi \in \Gamma\right\},
$$

где $\|\cdot\|$ - евклидова метрика на окружности. Можно сказать, что величина $\|\xi \cdot x\|$ измеряет, насколько $\xi$ отличается от тривиального характера $\xi_{0} \equiv 1$. Простые рассуждения, связанные с принципом Дирихле, позволяют доказать следующую лемму (см., например, [57]).

Лемма 1. Пусть $\mathbf{G}$ - компактная абелева группа $и|\Gamma|=d$. Тогда справедливо неравенство $\mu_{\mathbf{G}}(\mathscr{B}(\Gamma, \varepsilon)) \geqslant \varepsilon^{d}$.

В частности, множества Бора всегда непустые.

В дальнейшем нам понадобится понятие свертки двух функций $f, g \in L_{1}(\mathbf{G})$ :

$$
(f * g)(x)=\int_{\mathbf{G}} f(y) g(x-y) d \mu_{\mathbf{G}}(x) .
$$

Имеем (см., например, [77])

$$
\widehat{(f * g)}(\xi)=\hat{f}(\xi) \hat{g}(\xi)
$$

Операция свертки очень важна для аддитивных задач комбинаторной теории чисел. Действительно, пусть $A$ и $B$ - произвольные подмножества группы $\mathbf{G}$. Определим сумму (по Минковскому) этих множеств:

$$
A+B:=\{c \in \mathbf{G}: c=a+b, a \in A, b \in B\} .
$$

Пусть $f$ - характеристическая функция множества $A$, а $g$-характеристическая функция множества $B$. Легко видеть, что $(f * g)(x) \neq 0$ тогда и только тогда, когда $x \in A+B$. Аналогично определяется сумма нескольких множеств. Если 
в группе $(\mathbf{G},+)$ есть еще одна бинарная операция, скажем, умножение, то тогда можно определить и произведение множеств. В дальнейшем нам понадобится $A+B$ - сумма различных элементов множеств $A$ и $B$ :

$$
A \dot{+} B:=\{c \in \mathbf{G}: c=a+b, a \in A, b \in B, a \neq b\} .
$$

Обозначим через $A_{1} \dot{+} \cdots \dot{+} A_{d}$ множество, образованное суммой различных элементов из множеств $A_{1}, \ldots, A_{d}$. Множество, состоящее из суммы $d$ различных элементов множества $A$, обозначим через $d \dot{A}$.

Часто мы будем рассматривать ситуацию, когда абелева группа $\mathbf{G}$ - конечна. Хорошо известно, что в этом случае $\widehat{\mathbf{G}}=\mathbf{G}$. Обозначим через $N$ мощность группы G. Очень удобно определить преобразование Фурье функции $f$ формулой

$$
(\Phi f)(\xi)=\hat{f}(\xi)=\sum_{x \in \mathbf{G}} f(x) \overline{\xi(x)}
$$

Таким образом, в отличие от (1) мы не нормируем суммы по G. Нам понадобятся несколько известных формул, касающихся преобразования Фурье:

$$
\begin{gathered}
\|f\|_{2}^{2}:=\sum_{x \in \mathbf{G}}|f(x)|^{2}=\frac{1}{N} \sum_{\xi \in \widehat{\mathbf{G}}}|\hat{f}(\xi)|^{2}=\frac{1}{N}\|\hat{f}\|_{2}^{2} \quad \text { (равенство Парсеваля), (4) } \\
\langle f, g\rangle:=\sum_{x \in \mathbf{G}} f(x) \overline{g(x)}=\frac{1}{N} \sum_{\xi \in \widehat{\mathbf{G}}} \hat{f}(\xi) \overline{\hat{g}(\xi)}=\frac{1}{N}\langle\hat{f}, \hat{g}\rangle \quad \text { (тождество Планшереля), } \\
\sum_{x \in \mathbf{G}}|(f * g)(x)|^{2}=\sum_{x \in \mathbf{G}}\left|\sum_{y \in \mathbf{G}} f(y) g(x-y)\right|^{2}=\frac{1}{N} \sum_{\xi \in \widehat{\mathbf{G}}}|\hat{f}(\xi)|^{2}|\hat{g}(\xi)|^{2}, \\
f(x)=\frac{1}{N} \sum_{\xi \in \widehat{\mathbf{G}}} \hat{f}(\xi) \xi(x) \quad(\text { формула обращения), } \\
\widehat{f * g}=\hat{f} \hat{g} \quad \text { и } \quad(\widehat{f g})(x)=\frac{1}{N}(\hat{f} * \hat{g})(x) .
\end{gathered}
$$

Будем писать для краткости $\sum_{s}$ вместо суммы $\sum_{s \in \mathbf{G}}$ и $\sum_{\xi}$ вместо $\sum_{\xi \in \widehat{\mathbf{G}}}$.

Приведем два примера групп, которые будут особенно часто использоваться в нашем обзоре.

Пример 1. Пусть $N$ - натуральное число и $\mathbf{G}=\mathbb{Z} / N \mathbb{Z}=\mathbb{Z}_{N}$. Тогда все характеры - это корни из единицы степени $N$, т. е. $e^{2 \pi i r / N}$, где $r=0,1, \ldots, N-1$. Будем писать $e_{N}(x)$ или даже просто $e(x)$ вместо $e^{2 \pi i x / N}$. Тогда преобразование Фурье функции $f$ задается формулой

$$
(\Phi f)(\xi)=\hat{f}(\xi)=\sum_{x \in \mathbb{Z}_{N}} f(x) e(-\xi x) .
$$

В группе $\mathbb{Z}_{N}$ можно описать структуру множеств Бора (2). Простые рассуждения, связанные с принципом Дирихле, позволяют доказать следующую лемму. 
Лемма 2. Пусть $\mathbf{G}=\mathbb{Z}_{N} u|\Gamma|=d$. Тогда множество Бора $\mathscr{B}(\Gamma, \varepsilon)$ содержит арифметическую прогрессию длины $\varepsilon N^{1 / d}$.

Таким образом, множество Бора можно представлять себе как объединение арифметических прогрессий.

ПримеР 2. Пусть $p$ - простое число, $n$ - натуральное число и $\mathbf{G}=(\mathbb{Z} / p \mathbb{Z})^{n}$. Группа $\mathbf{G}$ является векторным пространством со скалярным произведением

$$
x \cdot y=\mathbf{x} \cdot \mathbf{y}=\langle\mathbf{x}, \mathbf{y}\rangle=x_{1} y_{1}+\cdots+x_{n} y_{n} \quad(\bmod p) .
$$

Преобразование Фурье функции $f: \mathbb{Z}_{p}^{n} \rightarrow \mathbb{C}$ вычисляется по формуле

$$
\hat{f}(r)=\sum_{x \in \mathbb{Z}_{p}^{n}} f(x) e(-\langle r, x\rangle),
$$

где $e(x)=e_{p}(x)=e^{2 \pi i x / p}$. Особенно просто устроено преобразование Фурье в группе $\mathbb{Z}_{2}^{n}$. Действительно, так как $e_{2}(x)=(-1)^{x}$, то для вещественной функции $f$ числа $\hat{f}(r)$ - вещественны.

В группах $\mathbb{Z}_{p}^{n}$ понятию множества Бора соответствует понятие афбинного подпространства. Пусть $\mathbf{v}_{1}, \ldots, \mathbf{v}_{k}$ - некоторые линейно независимые векторы, и пусть $\varepsilon_{1}, \ldots, \varepsilon_{k}-$ произвольные элементы $\mathbb{Z}_{p}$. Аффинным подпространством коразмерности $k$ называется множество

$$
P=P_{\varepsilon_{1}, \ldots, \varepsilon_{k}}\left(\mathbf{v}_{1}, \ldots, \mathbf{v}_{k}\right)=\left\{\mathbf{x} \in \mathbb{Z}_{p}^{n}:\left\langle\mathbf{x}, \mathbf{v}_{1}\right\rangle=\varepsilon_{1}, \ldots,\left\langle\mathbf{x}, \mathbf{v}_{k}\right\rangle=\varepsilon_{k}\right\} .
$$

Если все $\varepsilon_{i}$ равны нулю, то иногда мы будем писать $P\left(\mathbf{v}_{1}, \ldots, \mathbf{v}_{k}\right)$ вместо $P_{0, \ldots, 0}\left(\mathbf{v}_{1}, \ldots, \mathbf{v}_{k}\right)$. Для множества $W=\left(w_{1}, \ldots, w_{|W|}\right)$ пусть $P(W)$ означает $P\left(w_{1}, \ldots, w_{|W|}\right)$. Преобразование Фурье характеристической функции $\chi_{P}$ множества $P=P(W)$ вычисляются чрезвычайно просто. Пусть $L-$ линейное пространство размерности $k$, натянутое на векторы $\mathbf{v}_{1}, \ldots, \mathbf{v}_{k}$, и пусть $\mathbf{r} \in \mathbb{Z}_{p}^{n-}$ произвольный вектор. Ясно, что $P^{\perp}=L$ и для всякого $r \in L$ выполнено $\hat{\chi}_{P}(r)=|P|$. Далее, из равенства Парсеваля

$$
\sum_{r \in \mathbb{Z}_{p}^{n}}\left|\hat{\chi}_{P}(r)\right|^{2}=|P| N
$$

вытекает, что $\hat{\chi}_{P}(r)=0$ для любого $r \notin L$. Иными словами

$$
\hat{\chi}_{P}(r)=\chi_{L}(r)|P|,
$$

где $\chi_{L}-$ характеристическая функция множества $L$. Таким образом, $\left|\hat{\chi}_{P}(r)\right|$ либо нуль, либо равен $|P|$.

Сделаем еще несколько замечаний об обозначениях. Если $S \subseteq \mathbf{G}-$ некоторое множество, то $S(x)$ будем обозначать его характеристическую функцию. Иными словами, $S(x)=1$, если $x \in S$, и нуль иначе. Числа $\widehat{S}$ называют коэффициентами Фурье множества $S$. Символ $|S|$ используется для мощности множества $S$. Запись $\log$ означает логарифм по основанию два. Знаки « и 》 суть обычные символы Виноградова. Если $n$ - натуральное число, то будем 
писать $[n]$ для обозначения отрезка натурального ряда $\{1, \ldots, n\}$. Символ $\mathrm{E}(\cdot)$ обозначает математическое ожидание случайной величины, $\mathrm{P}(\cdot)$ - вероятность некоторого события. Пусть $\mathbb{D}$ - единичный диск на комплексной плоскости. Наконец, $\mathbb{Z}_{N}^{*}=\mathbb{Z}_{N} \backslash\{0\}$ и $(m, n)$ - наибольший общий делитель натуральных чисел $m$ и $n$.

\section{3. Арифметические прогрессии длины три}

В 1927 г. Б.Л. Ван дер Варден доказал свою знаменитую теорему об арифметических прогрессиях (см. [78]).

Теорема 1 (Ван дер Варден). Пусть $h u k$ - натуральные числа. Существует такое число $N(h, k)$, что для любого натурального $N \geqslant N(h, k)$ и произвольного разбиения множества $[N]$ на $h$ подмножеств одно из подмножеств содержит арифметическую прогрессию длины $k$, m. е. кортеж $n, n+d$, $n+2 d, \ldots, n+(k-1) d$, где $n, d-$ натуральные числа.

Пусть $N$ - натуральное число. Положим

$$
a_{k}(N)=\frac{1}{N} \max \{|A|: A \subseteq[N], A \text { не содержит }
$$

арифметических прогрессий длины $k\}$.

В [79] П. Эрдёш и П. Туран высказали гипотезу, согласно которой в любом множестве положительной плотности найдется арифметическая прогрессия заданной длины. Другими словами, они предположили, что для любого $k \geqslant 3$

$$
a_{k}(N) \rightarrow 0 \quad \text { при } N \rightarrow \infty .
$$

Ясно, что из этого предположения вытекает теорема Ван дер Вардена.

Гипотеза (10) в простейшем случае $k=3$ была доказана К. Ф. Ротом в [80]. В своей работе Рот, используя метод Харди-Литлвуда, получил следующий результат.

Теорема 2 (Рот). Для всех натуралъных $N \geqslant 3$ выполнено

$$
a_{3}(N) \ll \frac{1}{\log \log N} .
$$

Результат Рота был затем улучшен Е. Семереди в [81] и Д. Р. Хиф-Брауном в [82]. Независимо друг от друга оба этих автора получили следующую оценку для $a_{3}(N)$.

Теорема 3 (Семереди, Хиф-Браун). Пусть $N$-натуральное число, $N \geqslant 3$. Тогда

$$
a_{3}(N) \ll \frac{1}{(\log N)^{c}},
$$

где константу с можно взять равной $1 / 20$.

Наилучший, на сегодняшний день, результат об оценке сверху величины $a_{3}(N)$ принадлежит Ж. Бургейну [41] (см. также его более раннюю работу [40] и работу [83] по теореме Рота в $\mathbb{R}^{n}$ ). 
Теорема 4 (Бургейн). Пусть $N$ - натуралъное число, $N \geqslant 3$. Тогда

$$
a_{3}(N) \ll \frac{(\log \log N)^{3}}{(\log N)^{2 / 3}} .
$$

Для произвольного $k$ гипотеза (10) была доказана Е. Семереди [36], [84] в 1975 г. В своем доказательстве Семереди использует трудные комбинаторные аргументы. Основу его доказательства составляет так называемая лемма регулярности, которая является, на сегодняшний день, важнейшим инструментом исследования графов (см., например, [37], [85]). Альтернативное доказательство было предложено Х. Фюрстенбергом в [38]. Его подход использует методы эргодической теории. Фюрстенберг показал, что теорема Семереди эквивалентна утверждению о кратной возвращаемости для почти всех точек в произвольной динамической системе. Мы сформулируем его теорему в следующем виде.

ТеОрема 5 (Фюрстенберг). Пусть $X$ - метрическое пространство $c$ метрикой $d(\cdot, \cdot)$ и борелевской сигма-алгеброй измеримых множеств $\Phi$. Пусть $T$ сохраняющее меру $\mu$ отображение $X$ в себя $u k \geqslant 3$. Тогда для почти всех $x \in X$

$$
\liminf _{n \rightarrow \infty} \max \left\{d\left(T^{n} x, x\right), d\left(T^{2 n} x, x\right), \ldots, d\left(T^{(k-1) n} x, x\right)\right\}=0 .
$$

Следует отметить, что, используя свой метод, Фюрстенберг и его ученики получили множество глубоких обобщений теоремы Семереди (см., например, [24]-[35], [38], [39], [86]-[88]), которые комбинаторными методами доказать пока не удалось. Подробнее о теореме Семереди можно узнать в обзоре [4].

А. Беренд в работе [89] получил нижнюю оценку величины $a_{3}(N)$ (см. также статьи [90], [91]).

Теорема 6 (Беренд). Пусть $\varepsilon>0$ - действительное число. Тогда существует $N_{\varepsilon} \in \mathbb{N}$ такое, что для любого натурального $N, N \geqslant N_{\varepsilon}$, выполнено

$$
a_{3}(N) \geqslant \exp (-(1+\varepsilon) C \sqrt{\log N}),
$$

где $C>0$ - некоторая абсолютная константа.

Несмотря на свою простоту (доказательство занимает всего две страницы), результат А. Беренда, полученный им в 1946 г., на сегодняшний день остается наилучшим.

Р. Ранкин в [92] обобщил теорему Беренда на случай всех $k \geqslant 3$.

Теорема 7 (Ранкин). Пусть $\varepsilon>0$ - действительное число $u k \geqslant 3$ - натуральное. Тогда для всех достаточно больших $N$ выполнено

$$
a_{k}(N) \geqslant \exp \left(-(1+\varepsilon) C_{k}(\log N)^{1 /(k-1)}\right),
$$

где $C_{k}$ некоторая положительная эффективная константа, зависящая только om $k$.

$\mathrm{K}$ сожалению, методы Семереди дают очень слабые верхние оценки для $a_{k}(N)$. Эргодический подход вообще не дает никаких оценок. Только в 2001 г. В. Т. Гауэрс [8] получил количественный результат о скорости стремления к нулю величины $a_{k}(N)$ для $k \geqslant 4$. Он доказал следующую теорему. 
Tеорема 8 (Гауэрс). Пусть $k \geqslant 4$. Тогда

$$
a_{k}(N) \ll \frac{1}{(\log \log N)^{c_{k}}},
$$

где константа $c_{k}>0$ зависит только от $k$.

Результат Гауэрса является значительным шагом на пути к доказательству другой знаменитой гипотезы Эрдёша и Турана об арифметических прогрессиях.

ГиПотезА 1 (Эрдёш-Туран). Пусть $A=\left\{n_{1}<n_{2}<\cdots\right\}-$ бесконечная последовательность натуральных чисел такая, что

$$
\sum_{i=1}^{\infty} \frac{1}{n_{i}}=\infty
$$

Тогда А содержит арифметическую прогрессию любой длинъ.

Легко показать, что гипотеза 1 эквивалентна тому, что для всех натуральных $k \geqslant 3$ ряд $\sum_{l=1}^{\infty} a_{k}\left(4^{l}\right)$ сходится (доказательство этого факта может быть найдено, например, в [4]). Следовательно, из оценки величины $a_{k}(N)$ для всех $k \geqslant 3$ вида $a_{k}(N) \ll 1 /(\log N)^{1+\varepsilon}$, где $\varepsilon>0$, будет вытекать гипотеза 1 .

Мы обсудим теорему 8 в следующем разделе 4, а сейчас изложим доказательство теоремы 2. Согласно общей идеологии статьи [3] (см. также введение) рассмотрим сначала аналог результата Рота в группах $\mathbb{Z}_{p}^{n}$, где $p$ - простое число. Ясно, что понятие арифметической прогрессии длины три или, иными словами, трех точек $x, y, z$, удовлетворяющих уравнению $x+y=2 z$, бессмысленно в группе $\mathbb{Z}_{2}^{n}$. Поэтому простейшей из групп $\mathbb{Z}_{p}^{n}$, в которой можно рассмотреть задачу Рота, является группа $\mathbb{Z}_{3}^{n}$ (другие абелевы группы изучались в статьях [93]-[95]).

ТеОРема 9 (Рот, группа $\mathbb{Z}_{3}^{n}$ ). Пусть $\mathbf{G}=\mathbb{Z}_{3}^{n} u A$ - произвольное множество без арифметических прогрессий длины три. Тогда

$$
|A| \ll \frac{N}{\log N} .
$$

ДокАЗАТЕЛЬство. Нам понадобится довольно общее определение [7], [8].

ОПРЕДЕлЕниЕ 1. Пусть $\mathbf{G}$ - конечная абелева группа, $|\mathbf{G}|=N$, а $\alpha \in(0,1)$ действительное число. Функция $f: \mathbf{G} \rightarrow \mathbb{C}$ называется $\alpha$-равномерной, если

$$
\|\hat{f}\|_{\infty} \leqslant \alpha N
$$

Множество $A \subseteq \mathbf{G},|A|=\delta N$, называется $\alpha$-равномерным, если функция $f(x):=A(x)-\delta$ (балансовая функиия) является $\alpha$-равномерной.

Обсудим основные идеи доказательства теоремы 9. Предположим, что множество $A \subseteq \mathbb{Z}_{3}^{n},|A|=\delta N$, не содержит арифметических прогрессий длины три. Доказательство теоремы 9 представляет собой алгоритм. На первом шаге этого 
алгоритма возможны две ситуации: либо множество $A$ является $\alpha$-равномерным с некоторым $\alpha$, зависящим только от $\delta$ (в доказательстве $\alpha$ есть некоторая степень $\delta)$, либо не является.

Если $A$ есть $\alpha$-равномерное множество, то существование арифметических прогрессий в $A$ проверяется довольно просто (см. лемму 3 ниже). Пусть теперь множество $A$ не является $\alpha$-равномерным. Можно показать, что $\alpha$-равномерность множества $A$ эквивалентна равномерной распределенности $A$ в аффинных подпространствах коразмерности один. Говоря точнее, мощность пересечения $\alpha$-равномерного множества $A$ мощности $\delta N$ с любым таким подпространством $H$ примерно равна $\delta|H|$. Следовательно, если множество $A$ не является $\alpha$-равномерным, то найдется подпространство $H$ такое, что $|A \cap H|=(\delta+\theta)|H|$, где $|\theta|>0$. Более аккуратные рассуждения позволяют доказать, что подпространство $H$ можно выбрать так, чтобы величина $\theta$ была положительна и выражалась явным образом в терминах плотности $\delta$ (см. лемму 4 ).

После этого мы рассматриваем новое множество $A^{\prime}=A \cap H$ и применяем к нему наш алгоритм. Заметим, что так как $A^{\prime} \subseteq A$, то множество $A^{\prime}$ не содержит арифметических прогрессий длины три. Кроме того, плотность $A^{\prime}$ в $H$ не меньше, чем $\delta+\theta$, где $\theta>0$. Следовательно, на каждом шаге алгоритма плотность получающихся множеств увеличивается на положительную величину. С другой стороны, плотность всегда не превосходит единицы. Это означает, что через конечное число шагов наш алгоритм остановится. Следовательно, на каком-то шаге алгоритма мы получим подпространство $\widetilde{H}$ некоторой размерности и $\alpha$-равномерное множество $\widetilde{A}$, принадлежащее $A \cap \widetilde{H}$. Как было сказано выше, в этом случае множество $\widetilde{A}$ содержит арифметическую прогрессию длины три. Значит, множество $A$ также содержит арифметическую прогрессию. Опять получаем противоречие с предположением об отсутствии в $A$ арифметических прогрессий.

Мы привели схему доказательства теоремы 9. Приступим теперь к самому доказательству. Арифметическая прогрессия $\{x, x+d, x+2 d\}$ называется нетривиальной, если $d \neq 0$, и тривиалъной в противном случае.

ЛЕмма 3 ( $\alpha$-равномерный случай, произвольная группа без элементов порядка 2). Пусть $\mathbf{G}$ - конечная абелева группа, в которой отсутствуют элементы порядка два, $|\mathbf{G}|=N$ u $A, B, C \subseteq \mathbf{G}-$ любые множества, $|A|=\delta N$, $|B|=\beta N,|C|=\gamma N$. Если множество $A$ является $\delta(\beta \gamma)^{1 / 2} / 2$ - равномерным и $N>2 \beta^{-1} \gamma^{-1}$, то найдется решение уравнения $x+y=2 z, x \in A, y \in B$, $z \in C$, такое, что $x, y, z$ - различные.

Действительно, число решений уравнения $x+y=2 z, x \in A, y \in B, z \in C$, равно

$$
\sigma:=\sum_{x}(A * B)(2 x) C(x)
$$

или, в терминах преобразования Фурье,

$$
\sigma=\frac{1}{N} \sum_{r} \widehat{A}(r) \widehat{B}(r) \widehat{C}(-2 r)=\delta \beta \gamma N^{2}+\frac{1}{N} \sum_{r \neq 0} \widehat{A}(r) \widehat{B}(r) \widehat{C}(-2 r) .
$$


Пусть $\alpha=\delta(\beta \gamma)^{1 / 2} / 2$. Пользуясь $\alpha$-равномерностью множества $A$, неравенством Коши-Буняковского и равенством Парсеваля, находим

$$
\sigma \geqslant \delta \beta \gamma N^{2}-\alpha N(|B||C|)^{1 / 2} \geqslant \frac{\delta \beta \gamma N^{2}}{2}>|A|,
$$

и все доказано, поскольку число решений уравнения $x+y=2 z$ с $x=y=z$ не превосходит $|A|$.

Смысл леммы 3 чрезвычайно простой. Пусть, для простоты, $A=B=C$, $|A|=\delta N$. Тогда по лемме 3 любое $\delta^{2} / 2$-равномерное множество $A$ содержит нетривиальную арифметическую прогрессию. Теперь мы рассмотрим противоположную, в некотором смысле, ситуацию.

ЛЕмма 4 (не $\alpha$-равномерный случай, группа $\mathbb{Z}_{3}^{n}$ ). Пусть $A \subseteq \mathbb{Z}_{3}^{n}$ - произвольное множество, $|A|=\delta N$ и $\alpha \in(0,1]$ - действительное число. Если $A$ является не $\alpha$-равномерным, то существуют подпространство $Н$ коразмерности один и вектор $x$ такие, что $|A \cap(H+x)| \geqslant\left(\delta+2^{-1} \alpha\right)|H|$.

Действительно, так как множество $A$ не является $\alpha$-равномерным, то существует $r \neq 0$ такое, что $|\hat{f}(r)| \geqslant \alpha N$, где $f(x)=A(x)-\delta$. Пусть $H_{0}=\{x \in$ $\left.\mathbb{Z}_{3}^{n}:\langle x, r\rangle=0\right\}, H_{1}=\left\{x \in \mathbb{Z}_{3}^{n}:\langle x, r\rangle=1\right\}, H_{2}=\left\{x \in \mathbb{Z}_{3}^{n}:\langle x, r\rangle=-1\right\}$ и $a_{j}=\left\langle f, H_{j}\right\rangle, j \in[3]$. Тогда $\sum_{j} a_{j}=0$ и $\sum_{j}\left|a_{j}\right| \geqslant|\hat{f}(r)| \geqslant \alpha N$. Складывая последние два соотношения, находим $j_{0}$ такое, что $\left|a_{j_{0}}\right|+a_{j_{0}} \geqslant \alpha N / 3$. Отсюда $a_{j_{0}}>0$ и, более того, $a_{j_{0}} \geqslant \alpha N / 6$. Полагая $H=H_{0}$, для любого $x \in H_{j_{0}}$ получаем $a_{j_{0}}=|A \cap(H+x)|-\delta|H| \geqslant \alpha N / 6$, и лемма доказана.

Убедимся в справедливости теоремы 9. Объединим, для удобства, леммы 3,4 в одно предложение.

ПрЕДЛОЖЕНИЕ 1. Пусть $A \subseteq \mathbb{Z}_{3}^{n},|A|=\delta N,-$ произволъное множество безарифметических прогрессий длины три. Пусть также

$$
N>2 \delta^{-2} \text {. }
$$

Тогда существуют подпространство $H$ коразмерности один и вектор $x$ такие, что $|A \cap(H+x)| \geqslant\left(\delta+2^{-1} \delta^{2}\right)|H|$.

Покажем, как из предложения 1 вытекает теорема 9. Предположим, что множество $A \subseteq \mathbb{Z}_{3}^{n}$ не содержит арифметических прогрессий длины три. Как было сказано выше, доказательство теоремы 9 представляет собой алгоритм. Проведем первый шаг нашего алгоритма. Пусть $H_{0}=\mathbb{Z}_{3}^{n}, A_{0}=A, \delta_{0}=\delta$. Имеем $N>2 \delta^{-2}$. Применяя предложение 1 , получаем подпространство $H_{1}$ коразмерности один и вектор $x_{1}$, для которых выполнено $\left|A_{0} \cap\left(H_{1}+x_{1}\right)\right| \geqslant$ $\left(\delta_{0}+2^{-1} \delta_{0}^{2}\right)\left|H_{1}\right|$. После этого положим $A_{1}=\left(A_{0}-x_{1}\right) \cap H_{1}$.

Пусть на $i$-м шаге алгоритма, $i \geqslant 1$, мы построили подпространство $H_{i}$ коразмерности $i$, множество $A_{i} \subseteq H_{i}$, не содержащее арифметических прогрессий длины три, $\delta_{i}:=\left|A_{i}\right| /\left|H_{i}\right|$ и вектор $x_{i}$ такие, что

$$
\left|A_{i-1} \cap\left(H_{i}+x_{i}\right)\right| \geqslant\left(\delta_{i-1}+2^{-1} \delta_{i-1}^{2}\right)\left|H_{i}\right| .
$$


Заметим, что на каждом шаге алгоритма плотность множеств $A_{i}$ в подпространствах $H_{i}$ увеличивается на величину $2^{-1} \delta_{i-1}^{2} \geqslant 2^{-1} \delta^{2}$, поскольку на первом шаге алгоритма плотность $A_{0}$ в $H_{0}$ равна $\delta$. Если

$$
h_{i}:=\left|H_{i}\right|>2 \delta^{-2} \geqslant 2 \delta_{i}^{-2},
$$

то мы можем провести $(i+1)$-й шаг алгоритма. Легко видеть, что максимальное число шагов $K$ нашего алгоритма не превосходит $2 \cdot 2 \delta^{-1}=4 \delta^{-1}$, поскольку плотность множеств $A_{i}$ ограничена сверху единицей. Действительно, на переход от плотности $\delta$ до плотности $2 \delta$ необходимо затратить не более $2 \delta^{-1}$ шагов, поэтому всего нам требуется не более $2 \delta^{-1}+2(2 \delta)^{-1}+2(4 \delta)^{-1}+\cdots=4 \delta^{-1}$ шагов (более строго эти несложные вычисления изложены, например, в [8] или [4]). Это еще не есть доказательство теоремы 9, поскольку мы не проверяли выполнение условия (15) на каждом шаге нашего алгоритма. Имеем $\delta \gg 1 / \log N$. Отсюда

$$
h_{K}=3^{-K} N \geqslant 3^{-4 / \delta} N>2 \delta^{-2} .
$$

Так как $h_{i} \geqslant h_{K}$ для всех $i \leqslant K$, то неравенство (15) выполняется на каждом шаге алгоритма. Теорема доказана.

В работе [95] Т. Сандерс улучшил оценку $N / \log N$ в теореме 9 для группы $\mathbb{Z}_{4}^{n}$.

Теорема 10 (Сандерс). Пусть $\mathbf{G}=\mathbb{Z}_{4}^{n}$ u $A$ - произволъное множество без mроек $\{x, x+d, x+2 d\}$, где $2 d \neq 0$. Тогда

$$
|A| \ll \frac{N}{\log N \cdot(\log \log N)^{1 / 6-\varepsilon}},
$$

где $\varepsilon>0$ - произвольная постоянная.

Очень интересно получить какое-либо усиление оценки (12), поскольку это, по-видимому, позволит доказать новые оценки сверху для величины $a_{3}(N)$ в любой абелевой группе. Также интересно доказать гипотезу Эрдёша-Турана в группе $\mathbb{Z}_{4}^{n}$. Как легко видеть, для этого достаточно заменить константу $1 / 6$ в оценке (16) на любую величину, строго большую единицы.

Докажем теперь теорему 2. Мы используем несложную модификацию аргументов выше. Нам понадобится техническая лемма.

Лемма 5. Пусть $s, N$ - натуральные числа, $\eta \in(0,1]$ - действительное число, $\varphi: \mathbb{Z}_{N} \rightarrow \mathbb{Z}_{N}$ - линейная функция и $N \geqslant \eta^{-2} \cdot 2^{8}$. Тогда существует арифметическая прогрессия $P \subseteq \mathbb{Z}, 2^{-4} \eta s^{1 / 2} \leqslant|P| \leqslant 2^{-2} \eta s^{1 / 2}$, такая, что $\operatorname{diam}(\varphi(P)) \leqslant s u$

$$
[N]=\left(\bigsqcup_{l \in T}(P+l)\right) \sqcup \Omega,
$$

где $T$ - некоторое множество транслячий, $a|\Omega| \leqslant \eta N$.

ДокАЗАТЕЛЬСтво. Пусть $\varphi(x)=a x+b$. Если $a=0$, то лемма очевидна. Пусть $a \neq 0$. Кроме того, пусть $t=\left\lceil\eta N /\left(8 s^{1 / 2}\right)\right\rceil \geqslant 2$. Рассмотрим $t+1$ вычетов $0, a, 2 a, \ldots, t a \in \mathbb{Z}_{N}$. Разобьем отрезок $[1, N]$ на $t$ отрезков со стороной $(N-1) / t$. По принципу Дирихле в один из отрезков попадут два числа из 
нашей последовательности. Предположим, что в этот отрезок попали вычеты $t_{1} a, t_{2} a$, и пусть $t_{2}>t_{1}$. Положим $u=t_{2}-t_{1}$. Тогда $0<u \leqslant t$ и $|u a| \leqslant N / t$. Разобьем $[N]$ на классы вычетов $\mathscr{C}_{j}, j \in[u]$, по модулю $u$. Очевидно, что $\left|\mathscr{C}_{j}\right|=\lfloor(N-j) / u\rfloor+1$. Легко видеть, что последнее число есть либо $\lfloor N / u\rfloor$, либо $\lceil N / u\rceil$. Далее, $\mathscr{C}_{1}-$ наибольший по мощности класс и $\mathscr{C}_{1}=\lceil N / u\rceil$. Пусть $\mathscr{C}_{1}^{\prime}$ есть $\mathscr{C}_{1}$ без самой правой точки. Тогда $[N]=\left(\bigsqcup_{l=0}^{u-1}\left(\mathscr{C}_{1}^{\prime}+l\right)\right) \sqcup \Omega^{*}$, где $\left|\Omega^{*}\right| \leqslant u$.

Пусть $w=\lfloor s t / N\rfloor$. Имеем $w \leqslant s t / N \leqslant N /(4 u) \leqslant\lfloor N / u\rfloor / 2$. Следовательно, можно разбить любую арифметическую прогрессию $\mathscr{C}_{1}^{\prime}+l$ на сдвиги некоторой арифметической прогрессии $P,|P|=w$, и некоторую арифметическую прогрессию $\Omega_{l}$ с шагом $u$ и длиной не более $w$. Ясно, что

$$
w=|P| \geqslant \frac{s t}{2 N} \geqslant 2^{-4} \eta s^{1 / 2} .
$$

Тогда

$$
[N]=\left(\bigsqcup_{l=0}^{u-1} \bigsqcup_{l^{\prime}} P_{l, l^{\prime}}\right) \sqcup\left(\bigsqcup_{l=0}^{u-1} \Omega_{l}\right) \sqcup \Omega^{*},
$$

где $P_{l, l^{\prime}}-$ некоторые трансляции прогрессии $P$. Положим

$$
\Omega=\left(\bigsqcup_{l=0}^{u-1} \Omega_{l}\right) \sqcup \Omega^{*}
$$

Ясно, что $\Omega$ - отрезок вида $[q, N]$ и

$$
|\Omega| \leqslant 4 u w \leqslant \frac{8 u s t}{N} \leqslant \frac{8 s t^{2}}{N} \leqslant \eta N
$$

Кроме того,

$$
\operatorname{diam}\left(\varphi_{i}(P)\right) \leqslant|u r| \cdot|P| \leqslant \frac{N}{t} \frac{s t}{N}=s
$$

Лемма доказана.

Теперь мы можем установить аналог леммы 4.

ЛЕмма 6 (не $\alpha$-равномерный случай, группа $\mathbb{Z}_{N}$ ). Пусть $A \subseteq \mathbb{Z}_{N}$ - произвольное множество, $|A|=\delta N, \alpha \in(0,1]$ - действительное число $и N \geqslant$ $2^{12} \alpha^{-2}$. Если $A$ является не $\alpha$-равномерным, то существует прогрессия $Q$, $|Q| \geqslant 2^{-10} \alpha^{2} N^{1 / 2}$, такал, что $|A \cap Q| \geqslant\left(\delta+2^{-3} \alpha\right)|Q|$.

ДокАзАтельство. Действительно, поскольку множество $A$ не является $\alpha$-равномерными, то найдется $r \neq 0$ такое, что

$$
|\widehat{A}(r)|=\left|\sum_{x \in A} e^{-2 \pi i x r / N}\right| \geqslant \alpha N .
$$

Применим лемму 5 с параметрами $\eta=\alpha / 4, s=\lfloor\alpha N /(8 \pi)\rfloor \geqslant 1$. При этом линейное отображение $\varphi(x)$ равно $r x$. Тогда по неравенству треугольника

$$
\sum_{l=1}^{|T|}\left|\sum_{x \in P+l} f(x) e^{-2 \pi i x r / N}\right| \geqslant \frac{3 \alpha N}{4},
$$


где $f(x)=A(x)-\delta$. Пусть $x_{l}-$ произвольная точка в прогрессии $P+l$. Так как для любого $l \in[|T|]$ выполнено $\operatorname{diam}(\varphi(P+l)) \leqslant s$, то, применяя неравенство треугольника еще раз, получаем

$$
\begin{aligned}
\sum_{l=1}^{|T|}\left|\sum_{x \in P+l} f(x)\right| & =\sum_{l=1}^{|T|}\left|\sum_{x \in P+l} f(x) e^{-2 \pi i x_{l} r / N}\right| \\
& \geqslant \sum_{l=1}^{|T|}\left|\sum_{x \in P+l} f(x) e^{-2 \pi i x r / N}\right|-\sum_{l=1}^{|T|} \frac{\alpha|P|}{4} \geqslant \frac{\alpha N}{2} .
\end{aligned}
$$

Поскольку $\sum_{x} f(x)=0$, то

$$
\left|\sum_{l=1}^{|T|} \sum_{x \in P+l} f(x)\right| \leqslant|\Omega| \leqslant \frac{\alpha N}{4} .
$$

Следовательно,

$$
\sum_{l=1}^{|T|}\left(\left|\sum_{x \in P+l} f(x)\right|+\sum_{x \in P+l} f(x)\right) \geqslant \frac{\alpha N}{4},
$$

и, значит, для некоторого $l \in[|T|]$ имеем

$$
\sum_{x \in P+l} f(x)=|A \cap(P+l)|-\delta|P| \geqslant \frac{\alpha|P|}{8} .
$$

Положим $Q=P+l$. Тогда из леммы 5 вытекает оценка $|Q| \geqslant 2^{-4} \eta s^{1 / 2} \geqslant$ $2^{-10} \alpha^{2} N^{1 / 2}$ и лемма 6 полностью доказана.

Теперь мы можем получить теорему 2, используя те же рассуждения, что и при доказательстве теоремы 9. Пусть $A \subseteq[N],|A|=\delta N,-$ произвольное множество без арифметических прогрессий длины три. Можно считать, что $N-$ нечетное число. Пусть также $B=C=A \cap\{\lfloor N / 3\rfloor+1,\lfloor N / 3\rfloor+2, \ldots,\lfloor 2 N / 3\rfloor\}$. Отождествим множество $[N]$ с группой $\mathbb{Z}_{N}$. Тогда число решений уравнения $x+y \equiv 2 z(\bmod N)$, где $x \in A, y \in B$ и $z \in C$, совпадает с числом решений уравнения $x+y=2 z$. Как и выше, доказательство теоремы 2 представляет собой алгоритм, причем на каждом шаге алгоритма мы применяем к множествам $A, B, C$ лемму 3 , справедливую для любой абелевой группы без элементов порядка два, в частности для $\mathbb{Z}_{N}, N$ - нечетное, а если это невозможно (т. е. если множество $A$ не является $\alpha$-равномерным), то используем лемму 6 (детали можно найти в [8] или [4]). При этом мы получаем вложенные множества $A_{0}=A \supseteq A_{1} \supseteq A_{2} \supseteq \cdots$ и арифметические прогрессии $Q_{0}=[N] \supseteq Q_{1} \supseteq Q_{2} \supseteq \cdots$. Аналогично предыдущему, можно заметить, что алгоритм работает не более $K:=C \delta^{-1}$ шагов, где $C>0$ - некоторая абсолютная константа. Основное отличие теоремы 2 от теоремы 9 состоит в разном характере уменьшения длин прогрессий $Q_{i}$. Из леммы 6 вытекает, что на шаге $i$ выполнено

$$
\left|Q_{i}\right| \gg \delta^{4}\left|Q_{i-1}\right|^{1 / 2}
$$


В последней формуле стоит выражение $\delta^{4}$, поскольку, как легко видеть, величина $\alpha$ в теореме 2 , как и в теореме 9 , равна $\alpha=c \delta^{2}$, где $c>0$ - некоторая абсолютная константа. Нам необходимо проверить условие $\left|Q_{K}\right| \gg \delta^{-4}$ на последнем шаге алгоритма. С помощью метода математической индукции из оценки (18) можно вывести неравенство $\left|Q_{i}\right| \gg \delta^{4 i} N^{2^{-i}}$. По условию $\delta \gg 1 / \log \log N$. Следовательно,

$$
\left|Q_{K}\right| \gg \delta^{4 K} N^{2^{-K}} \geqslant \delta^{4 C / \delta} N^{2^{-C / \delta}} \gg \delta^{-4},
$$

и мы получаем теорему 2.

В работе [43] Б. Грин несколько усилил теорему Рота. Он доказал, что любое достаточно плотное подмножество $[N]$ содержит арифметическую прогрессию длины три, разность которой представляется в виде $x^{2}+y^{2}$, где $x, y$ - некоторые натуральные числа.

Теорема 11 (Грин). Пусть $N$ - натуральное число. Существует положительная эффективная константа $c>0$ такая, что всякое множество $A \subseteq[N],|A| \gg N /(\log \log N)^{c}$, содержит арифметическую прогрессию длины три, разность которой представима в виде $x^{2}+y^{2}$.

\section{4. Анализ Фурье высших порядков}

В предыдущем разделе мы доказали теорему Рота и упомянули теорему Гауэрса о множествах без арифметических прогрессий. Сформулируем эти результаты еще раз в терминах функций $a_{k}$.

Теорема 12 (Рот, Гауэрс). Для всех натуральных $N \geqslant 3 u k \geqslant 3$ выполнено

$$
a_{k}(N) \ll \frac{1}{\log \log N} .
$$

Как помнит читатель, доказательство теоремы Рота (случай $k=3$ ) представляло собой некоторую итерационную процедуру, причем на каждом шаге этой процедуры рассматривалось два варианта: первый, когда множество $A$ было $\alpha$-равномерным с достаточно малым $\alpha$, и второй, когда равномерности не было. Переформулируем основной результат, применявшийся в первом случае (см. доказательство леммы 3).

ПредлОЖениЕ 2 (равномерный случай). Пусть G - конечная абелева группа, в которой отсутствуют элементы порядка два, $|\mathbf{G}|=N u A \subseteq \mathbf{G}$, $|A|=\delta N$ - любое $\alpha$-равномерное множество. Тогда число арифметических прогрессий в множестве А равно

$$
\delta^{3} N^{2}+\theta \alpha \delta N^{2}
$$

əдe $|\theta| \leqslant 1$.

Пусть $f(x)=A(x)-\delta-$ балансовая функция множества $A$. Пусть также

$$
\Lambda_{3}(f)=\sum_{x, d \in \mathbf{G}} f(x) f(x+d) f(x+2 d),
$$


где $f$ - произвольная функция. Например, $\Lambda_{3}(A)$ - это число арифметических прогрессий длины три в множестве $A$. Легко видеть, что $\Lambda_{3}(A)=\delta^{3} N^{3}+\Lambda_{3}(f)$ и, следовательно, предложение 2 можно проинтерпретировать так:

$$
\text { норма }\|\hat{f}\|_{\infty} \text { мала } \Longrightarrow \Lambda_{3}(f) \text { мало. }
$$

Пусть теперь $A$ - множество без арифметических прогрессий длины $k, k>3$. Доказательство Гауэрса представляет собой глубокое обобщение метода Рота. Вместо величины $\Lambda_{3}$ он рассматривает ее аналог $\Lambda_{k}(f)=\sum_{x, d \in \mathbf{G}} f(x) \times$ $f(x+d) \cdots f(x+(k-1) d)$. Возникает вопрос: остается ли верной импликация (20), если вместо $\Lambda_{3}$ взять величину $\Lambda_{k}$ ? Как показал Гауэрс в работе [8], ответ здесь отрицательный (эргодический аспект этой проблемы был рассмотрен ранее в [87]). Контрпример очень простой. Пусть $\mathbf{G}=\mathbb{Z}_{N}, x \in \mathbb{Z}_{N}$ и $|x|$ означает расстояние от нуля до $x$ в приведенной системе вычетов. Тогда в группе $\mathbb{Z}_{N}$ можно взять, например, множество $A_{0}:=\left\{x \in \mathbb{Z}_{N}:\left|x^{2}\right| \leqslant 2^{-1} \delta N\right\}$. Пользуясь равномерной распределенностью квадратичных вычетов, легко видеть, что $\left|A_{0}\right| \sim \delta N$, величина $\|\hat{f}\|_{\infty}$ мала, но значение $\Lambda_{4}(f)$ велико (см. детали в [8]). Поэтому для задач с арифметическими прогрессиями длины больше, чем три, нам нужно как-то видоизменить импликацию (20).

Чтобы сформулировать правильный аналог (20), нам понадобятся нормы Гауэрса.

Пусть $\mathbf{G}$ - конечная абелева группа, $|\mathbf{G}|=N, d \geqslant 0$ - натуральное число и $\{0,1\}^{d}=\left\{\omega=\left(\omega_{1}, \ldots, \omega_{d}\right): \omega_{j} \in\{0,1\}, j=1, \ldots, d\right\}$ - обычный $d$-мерный куб. Для $\omega \in\{0,1\}^{d}$ пусть $|\omega|$ равно $\omega_{1}+\cdots+\omega_{d}$. Если $h=\left(h_{1}, \ldots, h_{d}\right) \in \mathbf{G}^{d}$, то $\omega \cdot h:=\omega_{1} h_{1}+\cdots+\omega_{d} h_{d}$. Пусть также $\mathscr{C}$ означает оператор комплексного сопряжения. Если $n$ - натуральное число, то $\mathscr{C}^{n}$ означает применение оператора комплексного сопряжения $n$ раз.

ОПределение 2. Пусть $f: \mathbf{G} \rightarrow \mathbb{C}$ - произвольная функция. Равномерная норма Гауэрса (или просто норма Гауэрса) этой функции задается формулой

$$
\|f\|_{U^{d}}:=\left(\frac{1}{N^{d+1}} \sum_{x \in \mathbf{G}, h \in \mathbf{G}^{d}} \prod_{\omega \in\{0,1\}^{d}} \mathscr{C}^{|\omega|} f_{\omega}(x+\omega \cdot h)\right)^{1 / 2^{d}} .
$$

Можно показать, что выражение (21) действительно задает норму для всех $d \geqslant 2$ (см. [8] или [4]). В частности, справедливо неравенство треугольника

$$
\|f+g\|_{U^{d}} \leqslant\|f\|_{U^{d}}+\|g\|_{U^{d}} .
$$

Нормы Гауэрса, как и $L_{p}$-нормы, удовлетворяют неравенству монотонности

$$
\|f\|_{U^{d-1}} \leqslant\|f\|_{U^{d}}
$$

для всех $d \geqslant 2$. Отметим еще одно комбинаторное свойство норм Гауэрса.

Пусть $h \in \mathbf{G}^{d}$. Набор из $2^{d}$ точек вида $\omega \cdot h=\omega_{1} h_{1}+\cdots+\omega_{d} h_{d}, \omega \in\{0,1\}^{d}$, называется $d$-мерным кубом. Пусть $A \subseteq \mathbf{G}$ - некоторое множество. Говорят, что $A$ содержит $d$-мерный куб, если $A$ содержит все точки этого куба. 
Используя неравенство Коши-Буняковского, легко показать, что любое множество $A \subseteq \mathbf{G}$ мощности $\delta N$ всегда содержит не менее $\delta^{2^{d}} N^{d+1}$ кубов размерности $d$, причем равенство достигается на "случайных подмножествах" $\mathbf{G}$, имеющих плотность $\delta$ (см. [8] или [4]). С другой стороны, справедлив следующий критерий (для простоты мы формулируем его в группе $\mathbb{Z}_{N}$ ).

Теорема 13 (комбинаторный смысл норм Гауэрса в $\mathbb{Z}_{N}$ ). Пусть $d \geqslant 2$, $A \subseteq \mathbb{Z}_{N},|A|=\delta N$ u $f-$ балансовая функиия множества $A$. Имеем $\|f\|_{U^{d}}=$ o(1) при $N \rightarrow \infty$ тогда и только тогда, когда $A$ содержит $\delta^{2^{d}} N^{d+1}+o\left(N^{d+1}\right)$ кубов.

Дальнейшие свойства норм Гауэрса могут быть найдены в недавней работе [9] (см. также [75], [76]).

Остановимся чуть подробнее на случае $d=2$. Пользуясь формулой обращения (7), легко видеть, что $U^{2}$-норма функции $f$ выражается в терминах преобразования Фурье:

$$
\|f\|_{U^{2}}=\left(\frac{1}{N^{4}} \sum_{r \in \mathbf{G}}|\hat{f}(r)|^{4}\right)^{1 / 4} .
$$

Таким образом, $U^{2}$-норма некоторой функции - это просто $L_{4}$-норма ее преобразования Фурье, умноженная на $N^{-1}$.

Лемма 7. Пусть $f: \mathbf{G} \rightarrow \mathbb{D}$ - произвольная функиия. Тогда $\|\hat{f}\|_{\infty} \leqslant\|\hat{f}\|_{4}$. Наоборот, $\|f\|_{U^{2}}=N^{-1}\|\hat{f}\|_{4} \leqslant\|\hat{f}\|_{\infty}^{1 / 2} N^{1 / 2}$.

Первое неравенство леммы очевидно, а второе вытекает из равенства Парсеваля

$$
\|\hat{f}\|_{4}^{4}=\sum_{r}|\hat{f}(r)|^{4} \leqslant\|\hat{f}\|_{\infty}^{2}\|\hat{f}\|_{2}^{2}=N\|\hat{f}\|_{\infty}^{2}\|f\|_{2}^{2} \leqslant\|\hat{f}\|_{\infty}^{2} N^{2} .
$$

Лемма 7 говорит о том, что для функций $f$, значения которых по модулю ограничены единицей, нет большой разницы между $\|\hat{f}\|_{\infty}$ и $\|f\|_{U^{2}}$. Поэтому импликацию (20) можно переформулировать так

$$
\|f\|_{U^{2}} \text { мало } \Longrightarrow \Lambda_{3}(f) \text { мало. }
$$

Теперь стало ясно, как можно обобщить импликацию (20).

УТВЕРЖДЕНИЕ 1 (см. [7], [8]). Для всех $k \geqslant 3$ выполнено

$$
\|\hat{f}\|_{U^{k-1}} \text { мало } \Longrightarrow \Lambda_{k}(f) \text { мало. }
$$

Итак, мы получили аналог предложения 2 или, что то же самое, леммы 3 для случая арифметических прогрессий длины $k, k>3$. Нам осталось найти правильный вариант леммы 6. Пользуясь неравенствами для норм из леммы 7 , переформулируем лемму 6 следующим образом.

ЛЕмма 8 (не равномерный случай, $U_{2}$-норма). Пусть $A \subseteq \mathbb{Z}_{N}-$ произвольное множество, $|A|=\delta N, f$ - балансовая функция множества $A, \alpha \in(0,1]$ действительное число и $N \gg \alpha^{-C}$. Если $\|f\|_{U^{2}} \geqslant \alpha$, то существует прогрессия $Q,|Q| \gg \alpha^{C} N^{1 / 2}$, такая, что $|A \cap Q| \geqslant\left(\delta+c \alpha^{C}\right)|Q|$. Здесъ $C, c>0-$ некоторые константы. 
Выше условие не $\alpha$-равномерности множества $A$ было заменено на оценку $\|f\|_{U^{2}} \geqslant \alpha$, что, конечно, можно сделать ввиду леммы 7. Сформулируем аналог леммы 8 для норм $U^{k}, k \geqslant 3$ (см. [8]).

ЛЕмма 9 (не равномерный случай, $U_{k}$-норма). Пусть $A \subseteq \mathbb{Z}_{N}-$ произвольное множество, $|A|=\delta N, f-$ балансовая функция множества $A, \alpha \in(0,1]-$ действительное число и $N \gg \alpha^{-C}$. Если $\|f\|_{U^{k}} \geqslant \alpha$, то существует прогрессия $Q,|Q| \gg \alpha^{C} N^{c \alpha^{C}}$, такая, что

$$
|A \cap Q| \geqslant\left(\delta+c \alpha^{C}\right)|Q| .
$$

Здесь с, C>0 - некоторые константы.

Заметим, что в лемме 9 , в отличие от леммы 8 , оценка на длину прогрессии $Q$ более слабая. В какой-то степени это по существу. По крайней мере, в примере с множеством $A_{0}$ (см. с. 143) нельзя найти прогрессию $Q$ в $\mathbb{Z}$ (или даже в $\mathbb{Z}_{N}$ ) длиной по порядку больше, чем $N^{1 / 2}$, для которой было бы выполнено неравенство (27).

Как мы видели выше (см. лемму 7), малость коэффициентов Фурье функции $f$ эквивалентна малости ее $U^{2}$-нормы. Это совершенно не так в случае $U^{k}$-норм, $k \geqslant 3$. Тот же самый пример Гауэрса множества $A_{0}$ показывает, что существуют множества с большой $U^{3}$-нормой их балансовой функции, но с малыми коэффициентами Фурье.

Дело в том, что даже в самом простом случае $k=4$ равномерная $U^{3}$-норма Гауэрса функции $f$ может быть выражена только через коэффициенты Фурье разностной функиии $\Delta(f ; u)(x):=f(x) \overline{f(x+u)}$ :

$$
\|f\|_{U^{3}}^{8}=\frac{1}{N^{6}} \sum_{u} \sum_{r}|\hat{\Delta}(f ; u)(r)|^{4} .
$$

Из этой формулы вытекает, что $U^{3}$-норма балансовой функции множества $A$ мала тогда и только тогда, когда для "почти всех" $u$ множество $A \cap(A-u)$ имеет маленькие коэффициенты Фурье (см. [7], [8] или обзор [4]). Исходя из такого выражения для $U^{3}$-нормы видим, что доказательство леммы 9 представляет значительные сложности даже в случае $k=4$, поскольку условие $\|f\|_{U^{3}} \geqslant \alpha$ дает нам информацию о разностных функциях, а не о самой функции $f$. Тем не менее, в статье [8] Гауэрс решил эту обратную задачу аддитивной теории чисел и получил следующую информацию об исходной функции $f$.

Tеорема 14 (Гауэрс). Пусть $A \subseteq \mathbb{Z}_{N}$ - некоторое множество, $f$ - его балансовая функция, $\eta>0$ - действительное число и $\|f\|_{U^{d}} \geqslant \eta$. Тогда найдутся абсолютные константы $C_{d}, c_{d}>0$ такие, что для всех $N \geqslant \exp \left(C_{d} \eta^{-C_{d}}\right)$ существуют многочлен $p_{d-1}(x)$ степени $d-1$ и арифметическая прогрессия $P$, $|P| \gg c_{d} \eta^{C_{d}} N^{c_{d} \eta^{C_{d}}}$, для которых выполнено

$$
\left|\sum_{x \in P} f(x) e\left(p_{d-1}(x)\right)\right| \geqslant c_{d} \eta^{C_{d}}|P| .
$$

Используя количественную версию теоремы А. Вейля о равномерной распределенности дробных долей многочленов, Гауэрс уже относительно просто 
вывел из теоремы 14 лемму 9. Можно даже сказать, что теорема 14 является ядром его доказательства. Возможно чуть обобщить теорему 14.

ТеОрема 15 (Гауэрс). Пусть $\eta>0$ - действительное число, $f: \mathbb{Z}_{N} \rightarrow \mathbb{D}$ некоторая функция, $\sum_{x} f(x)=0 u\|f\|_{U^{d}} \geqslant \eta$. Тогда найдутся абсолютные константы $C_{d}, c_{d}>0$ такие, что для всех $N \geqslant \exp \left(C_{d} \eta^{-C_{d}}\right)$ существуют многочлен $p_{d-1}(x)$ степени $d-1$ и арифметическая прогрессия $P$, $|P| \gg c_{d} \eta^{C_{d}} N^{c_{d} \eta^{C_{d}}}$, для которых выполнено

$$
\left|\sum_{x \in P} f(x) e\left(p_{d-1}(x)\right)\right| \geqslant c_{d} \eta^{C_{d}}|P| .
$$

Это утверждение называют слабой обратной теоремой для норм Гауэрса. "Слабость" здесь состоит в том, что, как мы видели ранее, в случае $d=2$ можно сказать гораздо больше:

$$
\begin{gathered}
\|f\|_{U^{2}} \geqslant \eta \quad \Longrightarrow \quad \text { существует многочлен } p_{1}(x), \operatorname{deg} p_{1}=1, \\
\text { такой, что }\left|\sum_{x} f(x) e\left(p_{1}(x)\right)\right| \gg \eta^{C} N,
\end{gathered}
$$

где $C>0$ - некоторая абсолютная константа. Иными словами, в последней формуле, в отличие от (29), отсутствует суммирование по прогрессии $P$. Слабая обратная теорема для норм Гауэрса в ситуации, когда $k=3$, была перенесена Грином и Тао на случай произвольной абелевой группы G (см. [9], а также [46]). Грубо говоря, для группы $\mathbf{G}$ арифметическая прогрессия $P$ в формуле (29) должна быть заменена на трансляцию множества Бора. Обратные теоремы, включающие в свою формулировку так называемые нильпоследовательности, были получены в статье [9]. О нильпоследовательностях см. в работах [28], [31]-[34], [71], [74]. Мы упомянем одну интересную гипотезу Грина-Тао, представляющую собой “сильную” версию теоремы 15, т. е. результат, не включающий в свою формулировку суммирование по прогрессии $P$. Пусть, для простоты, $\mathbf{G}=\mathbb{Z}_{5}^{n}$ и $k=4$.

ГиПотезА 2 (полиномиальная гипотеза для нормы Гауэрса). Пусть $\eta>0-$ действительное число, $f: \mathbb{Z}_{5}^{n} \rightarrow \mathbb{D}$ - некоторая фучниия, $\sum_{x} f(x)=0$ и $\|f\|_{U^{3}} \geqslant \eta$. Тогда найдутся абсолютные константы $C_{d}, c_{d}>0$ такие, что для всех $N \geqslant \exp \left(C_{d} \eta^{-C_{d}}\right)$ существуют матрица $M$ и вектор $r$, для которых выполнено

$$
\left|\sum_{x \in \mathbb{Z}_{5}^{n}} f(x) e(\langle x, M x\rangle+\langle r, x\rangle+b)\right| \geqslant c_{d} \eta^{C_{d}} N .
$$

Существует связь между некоторыми обратными задачами аддитивной комбинаторики и обратными проблемами теории норм Гауэрса (см. [96]). Гипотеза 2 вытекает из следующей гипотезы Каталины Мартон, которая сейчас называется гипотезой Фреймана-Ружи.

ГипотезА 3 (полиномиальная гипотеза Фреймана-Ружи). Пусть $A \subseteq \mathbb{Z}_{p}^{n}$, $p$ - простое число, $K \geqslant 1$ - действительное число и $|A+A| \leqslant K|A|$. Тогда 
существует множество $A^{\prime} \subseteq A,\left|A^{\prime}\right| \geqslant|A| / C_{1}(K)$, содержащееся в аффинном пространстве размера не больше $C_{2}(K)|A|$, причем функции $C_{1}(K), C_{2}(K)$ зависят от $K$ полиномиальным образом.

Гипотеза выше справедлива, если $C_{1}(K)$ и $C_{2}(K)$ зависят от $K$ экспоненциально (см., например, [9]). Совсем недавно в статье [72] была доказана эквивалентность гипотез 2 и 3 , а также эквивалентность соответствующих гипотез в $\mathbb{Z}_{N}$.

Доказательство теорем 8 и 15 содержит в себе много новых и красивых идей. Методы из работы Гауэрса развивались различными авторами (см., например, [9], [14], [15], [42]-[46]). Самым ярким из полученных в этих статьях результатом, является, несомненно, теорема Б. Грина и Т. Тао о прогрессиях в простых числах [42].

Теорема 16 (Грин, Тао). Для всех натуралъных $k \geqslant 3$ множество простых чисел содержит арифметическую прогрессию длины $k$.

На самом деле Грин и Тао доказали еще более сильный результат.

Пусть $A$ - произвольное подмножество множества простых чисел $\mathscr{P}$, и пусть $\pi(N)$ - число простых чисел, не превосходящих $N$. Верхней плотностью $A$ относительно $\mathscr{P}$ называется величина $\lim \sup _{N \rightarrow \infty}|A \cap[N]| / \pi(N)$.

Теорема 17 (Грин, Тао). Пусть $A \subseteq \mathscr{P}$ - произвольное множество положительной верхней плотности относительно $\mathscr{P}$, и пусть $k \geqslant 3$. Тогда $A$ содержит арифметическую прогрессию длины $k$.

В своем доказательстве Грин и Тао использовали некоторую "сильную" версию теоремы Гауэрса 15, правда, в их аналоге неравенства (29) вместо многочлена стояла некоторая более сложно устроенная "дуальная" функция [42].

Для $k=3$ теорему 16 доказал в 1938 г. Н. Г. Чудаков [97] (см. также [98], [99]), а теорему 17 - Грин в [100]. Грин получил даже более сильный результат. Пусть $\log _{[1]} N=\log N$ и для $l \geqslant 2$ положим $\log _{[l]} N=\log \left(\log _{[l-1]} N\right)$. Таким образом, $\log _{[l]} N$ есть результат взятия логарифма от числа $N l$ раз подряд.

ТеОрема 18 (Грин). Пусть $N$ - достаточно болъшое натуральное число и $A$ - произвольное подмножество $\mathscr{P} \cap[N]$ такое, что

$$
|A| \gg \frac{N \sqrt{\log _{[5]} N}}{\log N \cdot \sqrt{\log _{[4]} N}} .
$$

Тогда А содержит арифметическую прогрессию длины три.

Отметим, что из гипотезы 1 вытекают обе теоремы 16 и 17.

\section{5. Задачи о раскрасках}

Пусть $k$-натуральное число. Назовем $k$-раскраской множества целых чисел произвольное отображение $\chi: \mathbb{Z} \rightarrow\{1,2, \ldots, k\}$. При этом отрезок натурального ряда $\{1,2, \ldots, k\}$ мы ассоциируем с $k$ различными цветами. В том случае, когда значение величины $k$ не важно, мы просто будем говорить о конечных 
раскрасках. Если $f\left(x_{1}, \ldots, x_{n}\right)=0, x_{i} \in \mathbb{Z}$, некоторое уравнение, то решение $\left(x_{1}^{(0)}, \ldots, x_{n}^{(0)}\right)$ этого уравнения называется монохроматическим, если все $x_{i}^{(0)}$ раскрашены в один и тот же цвет, иными словами, существует $m \in\{1, \ldots, k\}$, для которого $\chi\left(x_{i}^{(0)}\right)=m, i=1, \ldots, n$. Вопросу поиска монохроматических решений различных уравнений посвящена обширная литература (см. [101]-[106]). Первый результат о раскрасках был доказан И. Шуром [60] в 1916 г.

ТЕОРемА 19 (Шур). Для любой конечной раскраски множества $\mathbb{Z}$ существует монохроматическое решение уравнения $x+y=z$, где $x, y, z-$ ненулевые.

Другой результат о раскрасках - это знаменитая теорема Б. Л. Ван дер Вардена (см. раздел 3), доказанная им в 1927 г. (см. [78]).

Теорема 20 (Ван дер Варден). Пусть $l$ u $k$-натуралъные числа. Для любой раскраски целых чисел в $k$ иветов найдется монохроматическая арифметическая прогрессия длины $l$.

Теорема Ван дер Вардена сыграла значительную роль в развитии двух разделов математики - аддитивной комбинаторики и комбинаторной эргодической теории (см., например, обзор [4]). Сама по себе теорема Ван дер Вардена является одним из фундаментальных результатов теории Рамсея [101]-[103], [107].

Классическим результатом, полностью отвечающим на вопрос, имеет ли данное линейное уравнение монохроматическое решение при произвольной раскраске, является теорема Радо [105] (см. также [108]). Пусть $U=\left(u_{i j}\right)-$ матрица размера $m \times n$, все элементы которой целые. Рассмотрим линейное уравнение

$$
\sum_{j=1}^{n} u_{i j} x_{j}=0, \quad i=1, \ldots, m .
$$

ОПРЕДЕЛЕНИЕ 3. Система уравнений (31) называется регулярной в $\mathbb{Z}$, если для любой раскраски $\mathbb{Z}$ в конечное число цветов найдется монохроматическое решение системы (31).

Заметим, что числа $x_{1}, \ldots, x_{n}$ не предполагаются различными.

Теорема 21 (Радо). Пусть $U=\left(u_{i j}\right)$-матрииа размера $m \times n$, все элементы которой целье. Система уравнений (31) является регулярной в $\mathbb{Z}$ тогда и только тогда, когда найдутся столбиь $c_{1}, \ldots, c_{n}$ и числа $k_{i}, 1 \leqslant k_{1}<\cdots<$ $k_{t}=n$, такие, что новые столбцы

$$
A_{i}=\sum_{j=k_{i-1}+1}^{k_{i}} C_{j}
$$

удовлетворяют следующим условиям:

1) $A_{1}$ является нулевым столбиом;

2) для $i=1, \ldots, t$ столбеи, $A_{i}$ есть линейная комбиначия $C_{1}, \ldots, C_{k_{i-1}}$ с рациональными коэфбициентами.

Матрицы, для которых выполнены условия 1) и 2) теоремы 21, будем называть регулярными. В случае $m=1$ теорема Радо записывается наиболее просто. 
ТЕОРема 22 (Радо). Пусть $n$ - натуральное число $и c_{1}, \ldots, c_{n}$ - ненулевые иелье числа. Система уравнений

$$
c_{1} x_{1}+\cdots+c_{n} x_{n}=0
$$

является регулярной в $\mathbb{Z}$ тогда и только тогда, когда найдется непустое множество $I \subseteq[n]$ такое, что сумма $\sum_{i \in I} c_{i}$ равна нулю.

Например, уравнения $x-2 y+z=0$ и $x+y-z=0$ являются регулярными, а уравнение $x+y-5 z=0$ - нет. Если $x-2 y+z=0$, то числа $x, y, z$ образуют арифметическую прогрессию. Заметим, что для этого уравнения теорема 22 тривиальна, поскольку можно взять $x=y=z=1$. Теорема Ван дер Вардена 20 для случая $k=3$ позволяет утверждать, что для всякой раскраски $\mathbb{Z}$ в конечное число цветов существуют различные $x, y, z$ одного цвета, для которых выполнено $x-2 y+z=0$.

Монохроматические решения линейных уравнений, а также систем линейных уравнений изучались не только в $\mathbb{Z}$, но и в других группах (см., например, [106]). С другой стороны, про монохроматические решения нелинейнъх уравнений известно довольно мало. Например, до сих пор нет ответа на вопрос, существует ли монохроматическое решение у уравнения $x^{2}+y^{2}=z^{2}$, а также на вопрос, для любой ли конечной раскраски $\mathbb{Z}$ найдутся $x+y, x y$ одного и того же цвета (см. [109], проблема 3). Неизвестно решение и более сложного вопроса: при всякой ли раскраске существуют $x, y, x+y, x y$, имеющие один и тот же цвет? Заметим, что наличие ненулевого монохроматического решения у уравнения $x y=z$, использующего одно только умножение, легко следует из теоремы Шура (достаточно рассмотреть множество $\left\{2^{n}\right\}_{n \in \mathbb{N}_{0}}$ ). По поводу монохроматических решений нелинейных уравнений, включающих умножение, см. работы [110]-[112]. К сожалению, в этих статьях разбираются уравнения, имеющие либо только умножение, либо только сложение. Поэтому было бы интересно иметь решение хотя бы одной задачи о раскрасках, не являющейся линейной по одной из операций. Впрочем, в этом разделе речь пойдет исключительно о линейных уравнениях.

Традиционно в задачах о раскрасках использовались, в основном, либо комбинаторные методы (см., например, [107] или [102]), либо подход, связанный с применением эргодической теории (см., например, [38]), либо же топологические методы, например, метод ультрафильтров [113], [114]. Совсем недавно Т. Сандерс [115] впервые применил для этого круга проблем гармонический анализ. Автор настоящего обзора, А. Самородинский и А. Фиш также независимо предложили метод, опирающийся на анализ Фурье, для решения задач о раскрасках (см. доказательство теоремы 24).

Прежде чем обсуждать имеющиеся здесь результаты, переформулируем теорему 19.

Теорема 23 (Шур). Пусть $k$ - натуральное число. Существует такое число $S(k)$, что для любого натурального $N \geqslant S(k)$ и произволъной раскраски множества $[N]$ в $k$ иветов найдется монохроматическое решение уравнения $x+y=z$. 
В теореме 23, в отличие от теоремы 19, речь идет о конечных множествах (замена множества целых чисел на натуральные совершенно не важна). По этой причине теорему 23 называют конечной версией теоремы 19. Пользуясь общим принципом компактности (см., например, [102] или [4]), легко показать, что эти результаты эквивалентны. О поведении функции $S(k)$ известно (см. [102]) только лишь, что

$$
k \ll \log S(k) \ll k \log k .
$$

Верхняя оценка в (33) довольно просто вытекает из неравенств для некоторых чисел Рамсея. Нижняя оценка вообще элементарна, мы приведем имеющуюся здесь конструкцию (оценка на величину $S(k)$ вида $\log S(k) \geqslant C k$ с наилучшей константой $C>1$ может быть найдена в [102]). Действительно, заметим, что среди нечетных чисел нет решений уравнения Шура $x+y=z$. Поэтому, взяв произвольный отрезок $[N]$ натурального ряда, мы раскрасим множество нечетных чисел в один и тот же цвет, скажем, цвет 1. Оставшееся множество четных чисел изоморфно отрезку $[N / 2]$. Поэтому раскрасим множество "нечетных чисел" в этом отрезке в другой цвет -2 . И так далее. В конце концов мы получим оценку $S(k) \gg 2^{k}$.

В случае, когда рассматриваются конечные версии задач Ван дер Вардена и Радо, возникают аналоги функции $S(k)$, а именно функции $W(k)$ и $R_{U}(k)$. Здесь $U$ - это матрица линейного уравнения (31). Про поведение этих функций известно довольно мало (см. [102]). Теорема 23 показывает нам, как можно сформулировать вопрос о монохроматических решениях линейного уравнения (31) в случае конечной абелевой группы. Для простоты мы разберем только случай $m=1$.

Пусть $c_{1}, \ldots, c_{l}$ - произвольные элементы группы G. Рассмотрим линейное уравнение

$$
c_{1} x_{1}+\cdots+c_{l} x_{l}=0 .
$$

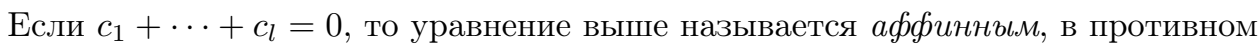
случае - неаффинным. Например, уравнение Шура - неаффинно, а задача Ван дер Вардена - аффинна. Множество $A$ содержит решение уравнения (34), если найдутся $x_{1}, \ldots, x_{l} \in A$, удовлетворяющие равенству (34).

ОПРЕДЕЛЕНИЕ 4. Рамсеевским числом уравнения (34) называют максимальное натуральное $R=R(\mathbf{c})$ такое, что при любом разбиении группы $\mathbf{G}$ на $R$ подмножеств одно из подмножеств содержит решение (34).

Проиллюстрируем применение метода анализа Фурье к задачам о раскрасках, предложенное Сандерсом. Мы рассмотрим наиболее простую ситуацию, когда $\mathbf{G}=\mathbb{Z}_{2}^{n}$.

Теорема 24 (Сандерс). Пусть $l$ - нечетное число и $\mathbf{c}=(1, \ldots, 1)$, причем число 1 присутствует в векторе с ровно l раз. Тогда

$$
R(\mathbf{c}) \geqslant C_{l} n^{1-1 /(l-1)},
$$

где $C_{l}>0$ - некоторая константа, зависящая только от $l$. Если же $l \gg \log n$, mo

$$
R(\mathbf{c}) \gg \frac{n}{\log n}
$$


ЗАмечАниЕ 1 . Имеющаяся в группе $\mathbb{Z}_{2}^{n}$ оценка сверху $R(\mathbf{c}) \leqslant n$ получается совершенно тривиально. Действительно, раскрасим любую гиперплоскость коразмерности один, не содержащую нуль, в цвет 1, затем возьмем оставшееся подпространство $H$ и заметим, что оно изоморфно $\mathbb{Z}_{2}^{n-1}$. Затем снова берем любую гиперплоскость в $H$ коразмерности один, не содержащую нуль, и раскрашиваем ее в цвет 2 , и т. д. Поскольку число $l$ нечетное, то в конце концов мы раскрасим $\mathbb{Z}_{2}^{n}$ в $n$ цветов без монохроматических решений. Иными словами, $R(\mathbf{c}) \leqslant n$.

Как и в случае теоремы 23, комбинаторные методы позволяют доказать, что $R(\mathbf{c}) \gg n / \log n$ (см. [102]). Таким образом, для $l \gg \log n$ эта оценка и неравенство (36) теоремы 24 совпадают. При малых $l$ комбинаторный метод работает лучше аналитического.

ДОКАЗАТЕЛЬСТво ТЕОРЕмЫ 24. Пусть $k=R(\mathbf{c})$ и множество $\mathbb{Z}_{2}^{n}$ разбито на $k$ множеств (цветов) $C_{1}, \ldots, C_{k}$. Нам надо убедиться в том, что найдется ненулевое монохроматическое решение уравнения

$$
x_{1}+\cdots+x_{l}=0 \text {. }
$$

Доказательство теоремы 24 представляет собой алгоритм. На произвольном шаге $s$ алгоритма строится некоторое подпространство $V^{s}$ коразмерности $s$. Далее, с каждым $C_{i}$ из набора цветов $C_{1}, \ldots, C_{k}$ мы будем ассоциировать вектор $x_{i}^{s}$. Кроме того, с множеством $C_{i}$ связано число $\left(\delta_{*}\right)_{i}^{s}-$ плотность $C_{i}$ в $V^{s}+x_{i}^{s}$ и число $\delta_{i}^{s}=\left|C_{i} \cap V^{s}\right| /\left|V^{s}\right|$. При этом алгоритм устроен так, что величины $\left(\delta_{*}\right)_{i}^{s}$ не уменьшаются, а подпространства $V^{s}$ вложены друг в друга: $V^{0} \supseteq V^{1} \supseteq \cdots$.

На нулевом шаге положим $V^{0}=\mathbb{Z}_{2}^{n}$ и $x_{1}^{0}=\cdots=x_{l}^{0}=0$. Далее, пусть алгоритм проработал $s$ шагов. Выберем цвет $C_{i}$ с максимальным значением $\delta_{i}^{s}$. Ясно, что $\delta_{i}^{s} \geqslant 1 / k$. Нам понадобится следующая лемма.

Лемма 10. Пусть $V \subseteq \mathbb{Z}_{2}^{n}$ - некоторое подпространство, $A \subseteq \mathbb{Z}_{2}^{n}-$ произвольное множество без ненулевых решений уравнения (37). Пусть тажже $x_{0} \in \mathbb{Z}_{2}^{n}$ - любой вектор, $\delta=|A \cap V| /|V|, \delta_{*}=\left|A \cap\left(V+x_{0}\right)\right| /|V|$. Наконеи, пусть $\delta_{*} \geqslant \delta u|V| \gg \delta^{-l}$. Тогда найдется $\xi \notin V^{\perp}$ такое, ито

$$
\left|\left(A \cap\left(V+x_{0}\right)\right)(\xi)\right| \geqslant\left(\delta_{*}+\delta_{*}\left(\delta \delta_{*}\right)^{1 /(2(l-2))}\right)|V| .
$$

Доказательство леммы представляет собой модификацию рассуждений из леммы 3. Вместо трех множеств в последней лемме надо просто рассмотреть $l$ множеств $A_{1}, A_{2}, \ldots, A_{l}$, а затем положить $A_{1}=A \cap V, A_{2}=\cdots=A_{l}=$ $A \cap\left(V+x_{0}\right)$. Так как характеристика группы $\mathbb{Z}_{2}^{n}$ равна двойке, то число решений уравнения (37), где $x_{i} \in A_{i}$, равно нулю (подробное доказательство см. в [115]).

Применим теперь лемму 10 к множеству $C_{i}$, а затем используем аналог леммы 4 в группе $\mathbb{Z}_{2}^{n}$. Мы найдем подпространство $H$ и вектор $x$ такие, что

$$
\left|C_{i} \cap(H+x)\right| \geqslant\left(\left(\delta_{*}\right)_{i}^{s}+\left(\delta_{*}\right)_{i}^{s}\left(\delta_{i}^{s}\left(\delta_{*}\right)_{i}^{s}\right)^{1 /(2(l-2))}\right)|H| .
$$


Положим $V^{s+1}=H, x_{i}^{s+1}=x$, а векторы $x_{j}^{s+1}, j \neq i$, выберем так, чтобы максимизировать величину $\left|C_{j} \cap\left(V^{s+1}+z\right)\right| /\left|V^{s+1}\right|$. Ясно, что тогда справедливо неравенство $\delta_{i}^{s+1} \geqslant \delta_{i}^{s}, i \in[k]$. Мы описали один шаг работы алгоритма. Будем применять его и далее.

Поскольку всегда $\left(\delta_{*}\right)_{i}^{s} \geqslant \delta_{i}^{s}$, то легко видеть, что наш алгоритм закончится самое большее через $O_{l}\left(k \cdot k^{1 /(l-2)}\right)$ шагов (см. аналогичные рассуждения в разделе 3$)$. Следовательно, если выполнено неравенство $k^{1+1 /(l-2)} \gg n$, то мы найдем монохроматическое решение уравнения (37). Вспоминая теперь, что $k=R(\mathbf{c})$, получаем оценку (35). Для доказательства неравенства (36) достаточно заметить, что в случае $l \gg \log n$ формула (40) переписывается в виде

$$
\left|C_{i} \cap(H+x)\right| \geqslant\left(\delta_{*}\right)_{i}^{s}(1+\lambda)|H|,
$$

где $\lambda>0$ - некоторая константа. Поэтому в этой ситуации алгоритм сделает не более $O(\log k)$ шагов. Отсюда вытекает неравенство (36). Теорема 24 полностью доказана.

Мы приведем другое доказательство неравенства (36) в духе рассуждений раздела 3. Единственное отличие нашего подхода от метода Рота состоит в том, что на каждом шаге итерационной процедуры требуется находить собственное, а не произвольное аффинное подпространство, в котором увеличивается плотность множества $A$, где $A$ не содержит решений уравнения (37). Для этого приходится следить за знаками коэффициентов Фурье. Нам понадобятся несколько простых лемм.

Лемма 11. Пусть $A \subseteq \mathbb{Z}_{2}^{n}$ - произвольное множество без решений уравнения (37). Тогда найдется $\xi \neq 0$ такое, что $|\widehat{A}(\xi)| \geqslant \delta(\delta / 4)^{1 /(l-2)} N$.

Доказательство леммы представляет собой модификацию рассуждений из леммы 3 или леммы 10.

Лемма 12. Пусть $A \subseteq \mathbb{Z}_{2}^{n}-$ произвольное множество без решений уравнения (37) и для всех $\xi \neq 0$ выполнено $|\widehat{A}(\xi)| \leqslant \delta(\delta / 4)^{1 /(l-2)} N$. Тогда множество

$$
R_{\text {huge }}:=\left\{\xi:|\widehat{A}(\xi)| \geqslant 2^{-1 /(4 l)}|A|\right\}
$$

имеет мощность либо 1 , либо 2.

Действительно, пусть $\widetilde{R}=R_{\text {huge }} \backslash\{0\}$. Так как множество $A$ не содержит решений уравнения (37), то

$$
0=\sum_{\xi} \widehat{A}^{l}(\xi)=|A|^{l}+\sum_{\xi: \widehat{A}(\xi)>0} \widehat{A}^{l}(\xi)+\sum_{\xi: \widehat{A}(\xi)<0} \widehat{A}^{l}(\xi)=|A|^{l}+\sigma^{+}+\sigma^{-} .
$$

Ввиду последней формулы

$$
2^{-1 / 4}|A|^{l}|\widetilde{R}| \leqslant \sum_{\xi \in \widetilde{R}}|\widehat{A}(\xi)|^{l}=\sigma^{+}+\left|\sigma^{-}\right|=|A|^{l}+2 \sigma^{+} \leqslant \frac{3}{2}|A|^{l} .
$$

Отсюда $|\widetilde{R}|<2$ и, поскольку всегда $0 \in R_{\text {huge }}$, мы имеем $\left|R_{\text {huge }}\right|=1$ или 2.

Из рассуждений ниже будет видно, что оба варианта $\left|R_{\text {huge }}\right|=1$ и $\left|R_{\text {huge }}\right|=2$ могут реализоваться. 
Лемма 13. Пусть $l \geqslant 5, A \subseteq \mathbb{Z}_{2}^{n}-$ произвольное множество без решений уравнения (37) и вектор $\xi_{0} \notin R_{\text {huge }}$ таков, что $\widehat{A}\left(\xi_{0}\right) \leqslant 0$ - минимальное. Тогда найдется $\xi \neq 0$ такое, что

$$
\widehat{A}(\xi) \geqslant \min \left\{\delta\left(\frac{\delta}{4}\right)^{1 /(l-4)} N, \frac{1}{80}\left|\widehat{A}\left(\xi_{0}\right)\right|\right\} .
$$

ДокАзАтЕльство. Если существует $\xi \neq 0$ такой, что $\widehat{A}(\xi) \geqslant \delta(\delta / 4)^{1 /(l-4)} N$, то лемма доказана. Поэтому будем считать, что справедливо обратное неравенство. Тогда по лемме 12 мощность множества $\widetilde{R}:=R_{\text {huge }} \backslash\{0\}$ равна 0 или 1 . По условию множество $A$ не содержит решений уравнения (37). Следовательно,

$$
\begin{aligned}
0 & =N \sum_{x_{1}+\cdots+x_{l}=0} A\left(x_{1}\right) \cdots A\left(x_{l-1}\right) A\left(x_{l}\right)(-1)^{\left\langle x, \xi_{0}\right\rangle}=\sum_{\xi} \widehat{A}^{l-1}(\xi) \widehat{A}\left(\xi+\xi_{0}\right) \\
& =|A|^{l-1} \widehat{A}\left(\xi_{0}\right)+|A| \widehat{A}^{l-1}\left(\xi_{0}\right)+\sum_{\xi \neq 0, \xi \neq \xi_{0}} \widehat{A}^{l-1}(\xi) \widehat{A}\left(\xi+\xi_{0}\right)=\sigma_{0}^{\prime}+\sigma_{0}^{\prime \prime}+\sigma_{1} .
\end{aligned}
$$

Так как $\xi_{0} \notin R_{\text {huge }}$, то $\left|\widehat{A}\left(\xi_{0}\right)\right| \leqslant 2^{-1 /(4 l)}|A|$. Следовательно,

$$
\sigma_{0}^{\prime}+\sigma_{0}^{\prime \prime} \geqslant\left(1-2^{-1 / 12}\right)|A|^{l-1}\left|\widehat{A}\left(\xi_{0}\right)\right| \geqslant \frac{1}{20}|A|^{l-1}\left|\widehat{A}\left(\xi_{0}\right)\right| .
$$

При выводе последней формулы мы воспользовались неравенством $l \geqslant 3$. Возвращаясь к тождеству (43) и используя оценку $|\widetilde{R}| \leqslant 1$, получаем

$$
\begin{aligned}
& -\frac{1}{20}|A|^{l-1} \widehat{A}\left(\xi_{0}\right) \leqslant \sum_{\xi \neq 0, \xi \neq \xi_{0}} \widehat{A}^{l-1}(\xi) \widehat{A}\left(\xi+\xi_{0}\right) \\
& =\sum_{\xi \neq 0, \xi \neq \xi_{0}, \xi \notin \widetilde{R}} \widehat{A}^{l-1}(\xi) \widehat{A}\left(\xi+\xi_{0}\right)+\sum_{\xi \neq 0, \xi \neq \xi_{0}, \xi \in \widetilde{R}} \widehat{A}^{l-1}(\xi) \widehat{A}\left(\xi+\xi_{0}\right) \\
& \leqslant\left(\max _{\xi \neq 0, \xi \neq \xi_{0}} \widehat{A}\left(\xi+\xi_{0}\right)\right)\left|\widehat{A}\left(\xi_{0}\right)\right| \sum_{\xi \neq 0, \xi \neq \xi_{0}}\left|\widehat{A}^{l-2}(\xi)\right|+|A|^{l-1} \max _{\xi \neq 0, \xi \neq \xi_{0}} \widehat{A}\left(\xi+\xi_{0}\right) .
\end{aligned}
$$

Вспоминая, что $\widehat{A}(\xi) \leqslant \delta(\delta / 4)^{1 /(l-4)} N$, и действуя как в доказательстве леммы 12, имеем

$$
\sum_{\xi \neq 0, \xi \neq \xi_{0}}\left|\widehat{A}^{l-2}(\xi)\right| \leqslant \frac{3}{2}|A|^{l-2} \leqslant 2|A|^{l-2} .
$$

Соединяя последнюю формулу с (44), (46), получаем оценку (42). Лемма 13 доказана.

Докажем, наконец, нашу основную лемму, которая интересна сама по себе.

Лемма 14. Пусть $l \geqslant 5$ и $A \subseteq \mathbb{Z}_{2}^{n}-$ произволъное множество без решений уравнения (37). Тогда имеет место альтернатива:

1) либо найдется $\xi \neq 0$ такое, что $\widehat{A}(\xi) \geqslant \frac{1}{80} \delta\left(\frac{\delta}{4}\right)^{1 /(l-4)} N$,

2) либо существуют подпространство $H$, codim $H=1, u$ вектор $x_{0} \notin H$ maкuе, что $A \subseteq H+x_{0}$. 
Пример раскраски выше (см. замечание 1, с. 151), показывающий, что в группе $\mathbb{Z}_{2}^{n}$ справедливо неравенство $R(\mathbf{c}) \leqslant n$, наглядно демонстрирует нам возможность второго варианта альтернативы. Ясно, как из леммы 14 вытекает неравенство (36). Действительно, пусть имеется разбиение группы $\mathbb{Z}_{2}^{n}$ на $k$ множеств (цветов). Возьмем цвет максимальной мощности $C$ и применим к нему лемму 14. Если выполнен пункт 1), то по аналогу леммы 4 в группе $\mathbb{Z}_{2}^{n}$ имеем, что для подпространства $L:=\{x:\langle\xi, x\rangle=0\}$ выполнено

$$
|C \cap L| \geqslant\left(\delta+\frac{1}{80}\left(\frac{\delta}{4}\right)^{1 /(l-4)}\right)|L| .
$$

Затем применяем лемму $14 \mathrm{k}$ множеству $C \cap L$. И так далее. Если на каком-то этапе справедлива вторая альтернатива, то подпространство $H$ раскрашено в $k-1$ цвет. Далее, возьмем в $H$ цвет максимальной мощности и действуем как раньше. По условию $l \gg \log n$, поэтому для каждого цвета первая альтернатива леммы может быть выполнена не более $O(\log k)$ раз (см. формулу $(40)$ в доказательстве теоремы 24). Поэтому всего наш алгоритм работает не более $O(k \log k)$ шагов, что и дает оценку (36).

Докажем лемму 14. Пусть ситуация 1) не имеет места. По лемме 11 существует $\xi \neq 0$ такой, что $|\widehat{A}(\xi)| \geqslant \delta(\delta / 4)^{1 /(l-2)} N \geqslant \delta(\delta / 4)^{1 /(l-4)} N$. Имеем $\widehat{A}(\xi)<0$. Обозначим множество таких $\xi$ через $R^{-}$. Предположим, что найдется $\xi_{0} \neq 0$, для которого выполнено $\xi_{0} \in R^{-}$и $\xi_{0} \notin R_{\text {huge }}$. Тогда по лемме 13 существует $\xi$ такой, что выполнена оценка (42) и, значит, справедлива первая альтернатива леммы 14 . Нам осталось рассмотреть случай, когда $R^{-}=R_{\text {huge }}$. Из лемм 11,12 вытекает, что $\left|R_{\text {huge }}\right|=2$. Пусть $R_{\text {huge }}=\{0\} \sqcup\left\{\xi_{0}\right\}$. Тогда для всех $\xi \neq 0, \quad \xi \neq \xi_{0}$ выполнено $|\widehat{A}(\xi)| \leqslant \delta(\delta / 4)^{1 /(l-4)} N$. Пусть $H=\left\{x:\left\langle x, \xi_{0}\right\rangle=0\right\}$, $H_{1}=\left\{x:\left\langle x, \xi_{0}\right\rangle=1\right\}=H+x_{0}$, где $x_{0} \notin H$. Пусть также $A_{0}=A \cap H$, $A_{1}=A \cap H_{1}$. Нам надо доказать, что множество $A_{0}$ - пустое. Положим $A^{\prime}=A_{1}-x_{0} \subseteq H$. Поскольку $\widehat{A}\left(\xi_{0}\right)=\left|A_{0}\right|-\left|A_{1}\right| \leqslant 2^{-1 /(4 l)}|A|$ и $l \gg \log n$, то для всех $\xi \in \bar{H}, \quad \xi \neq 0$ выполнено $\left|\widehat{A}^{\prime}(\xi)\right| \leqslant(\delta / 2)^{1 /(l-4)}\left|A_{1}\right|:=\varepsilon\left|A_{1}\right|$. Предположим, что множество $A_{0}$ непусто. Возьмем $z \in A_{0}$ и рассмотрим уравнение $x_{1}+\cdots+x_{l-1}+z=0, x_{i} \in A^{\prime}$. По условию множество $A$ не содержит решений уравнения (37) и число $l$ - нечетное. Следовательно,

$$
0=\sum_{\xi} \widehat{A}^{\prime}(\xi)^{l-1}(-1)^{\langle\xi, z\rangle} \geqslant\left|A_{1}\right|^{l-1}-\varepsilon^{l-3}\left|A_{1}\right|^{l-2}|H|>0 .
$$

Полученное противоречие доказывает лемму 14, а вместе с ней и неравенство (36).

Другое применение анализа Фурье к задачам о раскрасках см. в статье [116].

\section{6. Множества больших тригонометрических сумм}

Пусть $\mathbf{G}$ - конечная абелева группа и $A \subseteq \mathbf{G}$ - некоторое множество, $|A|=$ $\delta|\mathbf{G}|=\delta N$. В двух предыдущих разделах мы видели, что для подсчета комбинаторных конфигураций в множестве $A$ (таких как арифметические прогрессии, решения линейных уравнений и т.д.) важную роль играют большие 
коэффициенты Фурье множества $A$. Поэтому естественно рассмотреть следующий объект:

$$
\mathscr{R}_{\alpha}=\mathscr{R}_{\alpha}(A)=\{r \in \mathbf{G}:|\widehat{A}(r)| \geqslant \alpha N\},
$$

где $\alpha \in(0, \delta]$ - некоторый параметр. Такие множества называются множествами больших тригонометрических сумм (или, в терминологии книги [2], спектром множества $A$ ).

Для многих задач комбинаторной теории чисел важно знать структуру множества $\mathscr{R}_{\alpha}$ (см. [60]). Иными словами, какими нетривиальными свойствами обладают множества больших тригонометрических сумм? Ясно, что вопрос о строении $\mathscr{R}_{\alpha}$ относится к обратным задачам аддитивной теории чисел (см. [1]).

Перечислим простейшие свойства множества $\mathscr{R}_{\alpha}$. Из определения $\mathscr{R}_{\alpha}$ вытекает, что $0 \in \mathscr{R}_{\alpha}$ и $\mathscr{R}_{\alpha}=-\mathscr{R}_{\alpha}$ в том смысле, что если $r \in \mathscr{R}_{\alpha}$, то и $-r \in \mathscr{R}_{\alpha}$. Далее, из равенства Парсеваля (4) вытекает оценка мощности $\mathscr{R}_{\alpha}$, а именно $\left|\mathscr{R}_{\alpha}\right| \leqslant \delta / \alpha^{2}$. Существуют ли у множества $\mathscr{R}_{\alpha}$ еще какие-то нетривиальные свойства? Ответ на этот вопрос оказывается положительным, хотя первый общий результат о строении множеств $\mathscr{R}_{\alpha}$ был получен относительно недавно в работе М.-Ч. Чанг 2002 г. (см. [61]).

Теорема 25 (Чанг). Пусть $\delta, \alpha$-действительные числа, $0<\alpha \leqslant \delta \leqslant 1$, $A$ - произвольное подмножество $\mathbf{G}$ мощности $\delta N$ и множество $\mathscr{R}_{\alpha}$ определено равенством (47). Тогда найдется множество $\Lambda=\left\{\lambda_{1}, \ldots, \lambda_{|\Lambda|}\right\} \subseteq \mathbf{G}$,

$$
|\Lambda| \leqslant 2\left(\frac{\delta}{\alpha}\right)^{2} \log \frac{1}{\delta},
$$

такое, что всякий элемент $r$ множества $\mathscr{R}_{\alpha}$ представляется в виде

$$
r=\sum_{i=1}^{|\Lambda|} \varepsilon_{i} \lambda_{i}, \quad \varepsilon_{i} \in\{-1,0,1\} .
$$

Таким образом, теорема 25 утверждает, что множество $\mathscr{R}_{\alpha}$, которое априори может иметь мощность $\delta / \alpha^{2}$, обязательно содержится в "линейной оболочке" относительно маленького множества мощности $2(\delta / \alpha)^{2} \log (1 / \delta)$. Другими словами, если интерпретировать множество $\Lambda$ как "базис", то можно сказать, что $\mathscr{R}_{\alpha}$ имеет не слишком большую размерность.

Заметим, что для произвольной функции $f$ множество точек, где преобразование Фурье этой функции большое, совершенно не обязано иметь какую-либо структуру. В доказательстве теоремы 25 в [61] существенно использовалось, что преобразование Фурье берется от характеристической функиии.

Развивая подход из [117], [118] (см. также [119]), Чанг применила свой результат в новом доказательстве теоремы Фреймана [120] о множествах с маленькой суммой. Другие приложения теоремы 25 к задачам комбинаторной теории чисел см. в [61]-[66]. В нашем обзоре применения множеств больших тригонометрических сумм и, в частности, теоремы Чанг к задачам комбинаторной теории чисел будут обсуждаться в разделах 7 и 9.

В статье [66] (см. также [121]) были получены дальнейшие результаты о множествах больших тригонометрических сумм. В частности, в них была доказана следующая теорема. 
Теорема 26. Пусть $\delta, \alpha$ - действительные числа, $0<\alpha \leqslant \delta, A-$ произвольное подмножество $\mathbf{G}$ мощности $\delta N, k \geqslant 2$ - натуральное число и множество $\mathscr{R}_{\alpha}$ определено равенством (47). Пусть также $B \subseteq \mathscr{R}_{\alpha} \backslash\{0\}-$ произвольное множество. Тогда величина

$$
T_{k}(B):=\left|\left\{\left(r_{1}, \ldots, r_{k}, r_{1}^{\prime}, \ldots, r_{k}^{\prime}\right) \in B^{2 k}: r_{1}+\cdots+r_{k}=r_{1}^{\prime}+\cdots+r_{k}^{\prime}\right\}\right|
$$

не меньше, чем

$$
\delta \frac{\alpha^{2 k}}{\delta^{2 k}}|B|^{2 k}
$$

Покажем, что утверждение теоремы 26 нетривиально, когда параметр $\delta$ стремится к нулю при $N$, стремящемся к бесконечности. Рассмотрим простейший случай $k=2$. Пусть мощность множества $\mathscr{R}_{\alpha}$ по порядку равна $\delta / \alpha^{2}$. Тогда по теореме 26 количество решений уравнения

$$
r_{1}+r_{2}=r_{3}+r_{4}, \quad \text { где } r_{1}, r_{2}, r_{3}, r_{4} \in \mathscr{R}_{\alpha} \backslash\{0\},
$$

по порядку не меньше, чем $\delta / \alpha^{4}$. Среди этих решений существует три серии тривиальных решений. Первая серия: $r_{1}=r_{3}, r_{2}=r_{4}$, вторая: $r_{1}=r_{4}, r_{2}=r_{3}$, и, наконец, третья серия: $r_{1}=-r_{2}, r_{3}=-r_{4}$. Следовательно, у уравнения (52) существует не более $3\left|\mathscr{R}_{\alpha}\right|^{2}$ тривиальных решений. Так как мощность множества $\mathscr{R}_{\alpha}$ не превосходит $\delta / \alpha^{2}$, то величина $3\left|\mathscr{R}_{\alpha}\right|^{2}$ оценивается сверху числом $3 \delta^{2} / \alpha^{4}$. Мы видим, что эта величина меньше, чем $\delta / \alpha^{4}$, когда $\delta$ стремится к нулю. Таким образом, теорема 26 утверждает, что у уравнения (52) существуют нетривиальные решения. В этом смысле теорема 26 показывает, что множество $\mathscr{R}_{\alpha}$ обладает некоторой аддитивной структурой.

Интересно отметить, что если параметр $\delta$ не стремится к нулю при $N \rightarrow \infty$, то структура множества $\mathscr{R}_{\alpha}$ может быть достаточно произвольной (см. по этому поводу работы [122], [123]). Мы приведем один из имеющихся здесь результатов (см. [122], [123] или [124]).

Теорема 27 (Назаров). Пусть $\alpha_{r}, r \in \mathbb{Z}_{N},-$ произвольные неотрицательнъе числа такие, что $\sum_{r} \alpha_{r}^{2} \leqslant N / 1600$. Тогда найдется функиия $f: \mathbb{Z}_{N} \rightarrow$ $[0,1]$ такая, что $\|f\|_{1}=N / 2 u$

$$
|\hat{f}(r)| \geqslant \alpha_{r}\|f\|_{1}
$$

для всех $r \in \mathbb{Z}_{N}$.

Таким образом, теорема 27 говорит о том, что если $\delta$ не стремится к нулю (точнее, если $\delta=1 / 2$ ), то множество больших тригонометрических сумм может содержать в себе произвольное множество.

В доказательстве теоремы 25 Чанг использовала теорему Рудина [77] (также см. [125]) о диссоциативных подмножествах G. По определению, множество $\mathscr{D}=\left\{d_{1}, \ldots, d_{|\mathscr{D}|}\right\} \subseteq \mathbf{G}$ диссоциативно, если из равенства

$$
\sum_{i=1}^{|\mathscr{D}|} \varepsilon_{i} d_{i}=0 \quad(\bmod N),
$$

где $\varepsilon_{i} \in\{-1,0,1\}$, вытекает, что все $\varepsilon_{i}$ равны нулю. 
ТеОрема 28 (Рудин). Существует абсолютная константа $C>0$ такая, что для произвольного диссоциативного множества $\mathscr{D} \subseteq \mathbf{G}$, произвольных комплексных чисел $a_{n} \in \mathbb{C} u$ всех натуральных чисел $p \geqslant 2$ выполнено неравенство

$$
\frac{1}{N} \sum_{x \in \mathbf{G}}\left|\sum_{n \in \mathscr{D}} a_{n} e(n x)\right|^{p} \leqslant(C \sqrt{p})^{p}\left(\sum_{n \in \mathscr{D}}\left|a_{n}\right|^{2}\right)^{p / 2} .
$$

Доказательства теоремы 28 и теоремы Чанг могут быть также найдены в [124], [126]. Мы покажем, как из теоремы Рудина и теоремы 26 вытекает аналог теоремы 25, отличающийся от теоремы Чанг только лишь чуть более слабой оценкой на мощность множества $\Lambda$. Действительно, возьмем произвольное диссоциативное подмножество $\mathscr{R}_{\alpha}$. По теореме 26 множество $\mathscr{R}_{\alpha}$ имеет аддитивную структуру в том смысле, что любое его подмножество обладает большим числом решений уравнения из (50). Но, с другой стороны, как легко видеть, из теоремы Рудина вытекает, что число таких решений должно быть мало. Отсюда можно вывести, что мощность любого диссоциативного подмножества $\mathscr{R}_{\alpha}$ мала. Возьмем в качестве диссоциативного подмножества максимальное диссоциативное подмножество $\mathscr{R}_{\alpha}$ (его "базис"). Тогда легко видеть, что любой элемент $\mathscr{R}_{\alpha}$ может быть записан в виде $(49)$, причем число элементов в этом разложении мало. Приведем теперь строгое доказательство.

ПреДЛОЖенИЕ 3. Пусть $\delta, \alpha$-действительнъе числа, $0<\alpha \leqslant \delta \leqslant 1, A-$ произвольное подмножество $\mathbf{G}$ мощности $\delta N$ и множество $\mathscr{R}_{\alpha}$ определено равенством (47). Тогда найдется множество $\mathscr{D}=\left\{d_{1}, \ldots, d_{|\mathscr{D}|}\right\} \subseteq \mathbf{G}$, $|\mathscr{D}| \leqslant 2^{8} C^{2}(\delta / \alpha)^{2} \log (1 / \delta)$, такое, что вслкий элемент $r$ множества $\mathscr{R}_{\alpha}$ представляется в виде

$$
r=\sum_{i=1}^{|\mathscr{D}|} \varepsilon_{i} d_{i}
$$

где $\varepsilon_{i} \in\{-1,0,1\}$, а $C$ - абсолютная константа из неравенства Рудина (54).

ДокАзАТЕЛьство. Пусть $k=2\lceil\log (1 / \delta)\rceil$, и пусть $\mathscr{D} \subseteq \mathscr{R}_{\alpha}-$ максимальное диссоциативное подмножество $\mathscr{R}_{\alpha}$. Так как $\mathscr{D}$ - диссоциативное множество, то $0 \notin \mathscr{D}$. Применяя теорему 26, получаем оценку

$$
T_{k}(\mathscr{D}) \geqslant \frac{\delta \alpha^{2 k}}{2^{4 k} \delta^{2 k}}|\mathscr{D}|^{2 k}
$$

С другой стороны,

$$
T_{k}(\mathscr{D}) \leqslant C^{2 k} \cdot 2^{k} k^{k}|\mathscr{D}|^{k},
$$

где $C$ - абсолютная константа из теоремы 28. Действительно, пусть числа $a_{n}$ в формуле $(54)$ равны $\mathscr{D}(n)$, а $p=2 k$. Тогда левая часть в неравенстве $(54)$ есть $T_{k}(\mathscr{D})$, а правая равна $C^{2 k} \cdot 2^{k} k^{k}|\mathscr{D}|^{k}$. Имеем $k=2\lceil\log (1 / \delta)\rceil$. Применяя неравенства $(56)$ и $(57)$, находим $|\mathscr{D}| \leqslant 2^{8} C^{2}(\delta / \alpha)^{2} \log (1 / \delta)$. Так как $\mathscr{D}$ - максимальное диссоциативное подмножество $\mathscr{R}_{\alpha}$, то любой элемент $r$ множества $\mathscr{R}_{\alpha}$ представляется в виде $r=\sum_{i=1}^{|\mathscr{D}|} \varepsilon_{i} d_{i}(\bmod N)$, где $d_{i} \in \mathscr{D}$ и $\varepsilon_{i} \in\{-1,0,1\}$. Заметим, что оценка $|\mathscr{D}| \leqslant 2^{8} C^{2}(\delta / \alpha)^{2} \log (1 / \delta)$ отличается от аналогичной оценки в теореме Чанг лишь в константу раз. Предложение 3 доказано. 
Точно таким же способом (см. [121]) из теоремы 26 и обобщений неравенства Рудина можно вывести теорему Ж. Бургейна (см. [41]), которую тот использовал при доказательстве теоремы 4.

Теорема 29 (Бургейн). Пусть $d$ - натуральное число, $\delta, \alpha$-действительнъе числа, $0<\alpha \leqslant \delta \leqslant 1, A-$ произвольное подмножество $\mathbf{G}$ мощности $\delta N$ и множество $\mathscr{R}_{\alpha}$ определено равенством (47). Пусть также $\Lambda$ - диссоциативное множество. Тогда для всех $d \geqslant 1$ выполнено

$$
\left|d \dot{\Lambda} \cap \mathscr{R}_{\alpha}\right| \leqslant 8\left(\frac{\delta}{\alpha}\right)^{2} \log ^{d}\left(\frac{1}{\delta}\right) .
$$

Ясно, что теорема 29 обобщает теорему 25. Неравенство (58) было чуть усилено в статье [121] для группы $\mathbb{Z}_{2}^{n}$.

Если не обращать внимания на мультипликативные константы, то, как показал Б. Грин в статье [127], теорема Чанг является точной. Развивая его метод в работе [128], автор доказал теорему, в некотором смысле обратную к теореме 25. Мы сформулируем ее в простейшей форме.

Теорема 30 (Грин, Шкредов). Пусть $\delta \leqslant 1 / 8$ и $\alpha$-действительные числа, $20 N^{-1 / 2}<\alpha \leqslant \delta / 32$ и $\Lambda$ - произвольное множество,

$$
|\Lambda| \leqslant 2^{-11}\left(\frac{\delta}{\alpha}\right)^{2} \log \left(\frac{1}{\delta}\right)
$$

являющееся диссоциативным. Тогда найдется множество $A \subseteq \mathbb{Z}_{N},|A|=$ $\lfloor\delta N\rfloor$, maкoе, чmo $\mathscr{R}_{\alpha}(A)=\{0\} \sqcup \Lambda \sqcup(-\Lambda)$.

Таким образом, теорема 30 утверждает, что любое диссоциативное множество $\Lambda$ “допустимой" мощности, т. е. такое, для которого выполнено неравенство (59), является вместе с $(-\Lambda)$ и нулем множеством больших тригонометрических сумм некоторого множества $A$. Отсюда, в частности, следует, что оценка (48) является точной по порядку. Этого нельзя сказать о числе слагаемых в представлении (49). Так, в работе [66] был доказан следующий результат.

Теорема 31. Пусть $N$ - натуральное число, $(N, 2)=1, \delta, \alpha$ - действительные числа, $0<\alpha \leqslant \delta \leqslant 1 / 16, A-$ произвольное подмножество $\mathbb{Z}_{N}$ мощности $\delta N$ и множество $\mathscr{R}_{\alpha}$ определено равенством (47). Тогда существует множество $\Lambda^{*} \subseteq \mathbb{Z}_{N}$,

$$
\left|\Lambda^{*}\right| \leqslant \max \left\{2^{12}\left(\frac{\delta}{\alpha}\right)^{2} \log \left(\frac{1}{\delta}\right), 2^{6} \log ^{2}\left(\frac{1}{\delta}\right)\right\},
$$

такое, что для любого вычета $r \in \mathscr{R}_{\alpha}$ существует набор $\lambda_{1}^{*}, \ldots, \lambda_{M}^{*}$ из не более чем $8 \log (1 / \delta)$ элементов $\Lambda^{*}$ такой, что

$$
r=\sum_{i=1}^{M} \varepsilon_{i} \lambda_{i}^{*}(\bmod N)
$$

əде $\varepsilon_{i} \in\{-1,0,1\}$. 
В этой же статье был получен аналогичный результат для подмножеств группы $\mathbb{Z}_{2}^{n}$. Легко видеть, что число элементов $\lambda_{i}^{*}$ в представлении (61) не может быть уменьшено. Действительно, пусть $\alpha \approx \delta$, и пусть $A$ - подмножество $\mathbb{Z}_{2}^{n}$ со свойством $\left|\mathscr{R}_{\alpha}(A)\right| \approx \delta / \alpha^{2} \approx 1 / \delta$. Такие множества $A$ существуют, например, можно взять в качестве $A$ любое подпространство $\mathbb{Z}_{2}^{n}$ мощности $\delta N$. Тогда по теореме Чанг найдется множество $\Lambda^{*},\left|\Lambda^{*}\right| \ll \log (1 / \delta)$, такое, что для любого элемента $\mathbf{r} \in \mathscr{R}_{\alpha}(A)$ справедливо представление (61). Так как $\left|\mathscr{R}_{\alpha}(A)\right| \approx 1 / \delta$, то легко видеть, что существует вектор $\mathbf{r} \in \mathscr{R}_{\alpha}(A)$ такой, что для его представления в виде $(61)$ необходимо $k \gg \log (1 / \delta)$ векторов. Действительно, так как линейных комбинаций, натянутых на произвольные $k$ векторов из $\Lambda^{*}$, не более $2^{k}\left(\begin{array}{c}\left|\Lambda^{*}\right| \\ k\end{array}\right)$, то должно выполняться неравенство $2^{k}\left(\begin{array}{c}\left|\Lambda^{*}\right| \\ k\end{array}\right) \gg 1 / \delta$, из которого и вытекает оценка $k \gg \log (1 / \delta)$.

В [23], [18] использовались другие аддитивные свойства множеств $\mathscr{R}_{\alpha}$.

ПреДЛОЖЕНИЕ 4. Пустъ $\delta, \alpha$-действителъные числа, $0<\alpha \leqslant \delta \leqslant 1, A-$ произвольное подмножество $\mathbf{G}$ мощности $\delta N$. Тогда для любого непустого множества $B \subseteq \mathscr{R}_{\alpha}$ выполнено

$$
\sum_{z}\left(B * B^{c}\right)(z) \mathscr{R}_{\alpha^{2} /(2 \delta)}(z) \geqslant \frac{\alpha^{2}}{2 \delta}|B|^{2} .
$$

ДокАзАТЕльство. Действительно, по определению множества $\mathscr{R}_{\alpha}$ получаем

$$
\alpha N|B| \leqslant \sum_{r \in B}|\widehat{A}(r)|=\sum_{x} A(x) \sum_{r} B(r) e^{-i \arg \widehat{A}(r)} e(-r x) .
$$

Применяя неравенство Коши-Буняковского и неравенство треугольника, находим

$$
\begin{aligned}
\frac{\alpha^{2}}{\delta}|B|^{2} N & \leqslant \sum_{x \in A}\left|\sum_{r} B(r) e^{-i \arg \widehat{A}(r)} e(-r x)\right|^{2} \\
& =\sum_{r, r^{\prime}} B(r) B\left(r^{\prime}\right) e^{-i \arg \widehat{A}(r)+i \arg \widehat{A}\left(r^{\prime}\right)} \widehat{A}\left(r-r^{\prime}\right) \leqslant \sum_{z}\left(B * B^{c}\right)(z)|\widehat{A}(z)| .
\end{aligned}
$$

Суммируя в последней формуле только по $z \in \mathscr{R}_{\alpha^{2} /(2 \delta)}$, получаем (62). Предложение 4 доказано.

Работы [8], [23] еще будут обсуждаться в разделе 9. Вопрос о строении множеств больших тригонометрических сумм в случае $\mathbf{G}=\mathbb{Z}$ был изучен Ж. Бургейном в [41]. Приложение этих результатов к теореме Фреймана о множествах с малым удвоением см. в [65].

Следует отметить, что вопрос о структуре множества $\mathscr{R}_{\alpha}$ в случае, когда параметр $\alpha$ близок к $\delta$, изучался гораздо ранее работы Чанг в статье [129], см. также обзор [130]. Аддитивная структура множеств больших тригонометрических сумм в этом случае очень наглядно демонстрируется следующей теоремой (см. также [131]).

ТЕОРема 32 (Юдин). Пусть $A \subseteq \mathbf{G}-$ произвольное множество, $|A|=\delta|\mathbf{G}|$ $u \varepsilon, \varepsilon^{\prime} \in(0,1)$ - любые числа. Тогда

$$
\mathscr{R}_{\delta(1-\varepsilon)}(A)+\mathscr{R}_{\delta(1-\varepsilon)}(A) \subseteq \mathscr{R}_{\delta\left(1-2\left(\varepsilon+\varepsilon^{\prime}\right)\right)}(A) .
$$


ДоказАтельство. Пусть $r \in \mathscr{R}_{\delta(1-\varepsilon)}(A)$ и $r^{\prime} \in \mathscr{R}_{\delta\left(1-\varepsilon^{\prime}\right)}(A)$. Ясно, что найдутся углы $\varphi$ и $\varphi^{\prime}$ такие, что $\operatorname{Re}\left(\widehat{A}(r) e^{i \varphi}\right) \geqslant(1-\varepsilon)|A|$ и $\operatorname{Re}\left(\widehat{A}\left(r^{\prime}\right) e^{i \varphi^{\prime}}\right) \geqslant(1-\varepsilon)|A|$. Отсюда

$$
\begin{aligned}
\operatorname{Re} \sum_{x \in A}\left(2 e^{i \varphi} e(r x)+2 e^{i \varphi^{\prime}} e\left(r x^{\prime}\right)-3\right) & =\operatorname{Re}\left(2 \widehat{A}(r) e^{i \varphi}+2 \widehat{A}\left(r^{\prime}\right) e^{i \varphi^{\prime}}-3\right) \\
& \geqslant\left(1-2\left(\varepsilon+\varepsilon^{\prime}\right)\right)|A| .
\end{aligned}
$$

Если мы покажем, что для всякого $x$ выполнено

$$
\operatorname{Re}\left(2 e^{i \varphi} e(r x)+2 e^{i \varphi^{\prime}} e\left(r^{\prime} x\right)-3\right) \leqslant \operatorname{Re}\left(e^{i \varphi+i \varphi^{\prime}} e\left(\left(r+r^{\prime}\right) x\right)\right),
$$

то включение $r+r^{\prime} \in \mathscr{R}_{\delta\left(1-2\left(\varepsilon+\varepsilon^{\prime}\right)\right)}(A)$ будет доказано. Зафиксируем $x \in \mathbf{G}$, и пусть $e^{i \varphi} e(r x)=e^{i \beta}, e^{i \varphi^{\prime}} e\left(r^{\prime} x\right)=e^{i \beta^{\prime}}$, где $\beta, \beta^{\prime} \in(-\pi / 2, \pi / 2]$. Тогда нам достаточно проверить, что

$$
2 \cos \beta+2 \cos \beta^{\prime}-3 \geqslant \cos \left(\beta+\beta^{\prime}\right) .
$$

Пользуясь выпуклостью вверх косинуса на интервале $(-\pi / 2, \pi / 2]$, находим

$$
\begin{aligned}
2 \cos \beta+2 \cos \beta^{\prime}-3 & \geqslant 4 \cos \frac{\beta+\beta^{\prime}}{2}-3 \\
& =2 \cos ^{2} \frac{\beta+\beta^{\prime}}{2}-1-2\left(1-\cos \frac{\beta+\beta^{\prime}}{2}\right)^{2} \geqslant \cos \left(\beta+\beta^{\prime}\right) .
\end{aligned}
$$

Теорема доказана.

Мы закончим этот раздел обсуждением вопроса, насколько малым может быть коэффициент Фурье характеристической функции. Эта задача была впервые рассмотрена в работе [132]. Наше изложение следует статье [131].

Теорема 33 (Конягин-Лев). Пусть $p$ - простое число и $\mathbf{G}=\mathbb{Z}_{p}$. Тогда:

1) для любого множества $A,|A|=n, 3 \leqslant n \leqslant p-1$, выполнено

$$
\min _{r \in \mathbf{G}}|\widehat{A}(r)|>n^{-(p-3) / 4}
$$

2) с другой стороны, для всякого $n$ вида $n=2^{k}<p / 20$, где $k$-натуральное число, найдется множество $A \subseteq \mathbf{G}$ такое, что

$$
|\widehat{A}(r)|<n^{-(\ln p) /(2 \ln 2)} .
$$

Мы докажем первый пункт теоремы 33. Пункт 2) проверяется достаточно просто - в качестве множества $A$ берется некоторое диссоциативное множество.

Легко видеть, что коэффициенты Фурье характеристической функции множества $A$ - ненулевые. Действительно, достаточно заметить, что коэффициенты Фурье суть значения многочлена $\widehat{A}(z)$ от корней из единицы и что многочлен $\widehat{A}(z)$ не делится на минимальный многочлен $1+z+\cdots+z^{p-1}$. Произведение $m:=\prod_{r \in \mathbb{Z}_{p}^{*}} \widehat{A}(r)$ является нормой любого корня полинома $\widehat{A}(z)$. В частности, 
$\prod_{r \in \mathbb{Z}_{p}^{*}} \widehat{A}(r) \in \mathbb{Z} \backslash\{0\}$. Из последнего соображения, равенства Парсеваля, а также неравенства между средним арифметическим и средним геометрическим получаем

$$
\begin{aligned}
1 & \leqslant \prod_{r \in \mathbb{Z}_{p}^{*}}|\widehat{A}(r)|^{2}=\left|\widehat{A}\left(r_{0}\right)\right|^{4} \prod_{r \in \mathbb{Z}_{p}^{*}, r \neq \pm r_{0}}|\widehat{A}(r)|^{2} \\
& \leqslant\left|\widehat{A}\left(r_{0}\right)\right|^{4}\left(\frac{1}{p-3} \sum_{r \in \mathbb{Z}_{p}^{*}, r \neq \pm r_{0}}|\widehat{A}(r)|^{2}\right)^{p-3} \\
& <\left|\widehat{A}\left(r_{0}\right)\right|^{4}\left(\frac{n(p-n)}{p-3}\right)^{p-3} \leqslant n^{p-3}\left|\widehat{A}\left(r_{0}\right)\right|^{4}
\end{aligned}
$$

и все доказано в случае $r_{0} \neq 0$. Если $r_{0}=0$, то неравенство (64) очевидно.

Достаточно интересно уменьшить зазор между верхней и нижней оценкой в теореме 33 .

\section{7. Комбинаторные конфигурации в суммах множеств}

В этом разделе мы рассмотрим задачи об арифметических прогрессиях в суммах, т. е. в множествах вида $A_{1}+\cdots+A_{k}$. Оказывается, такие множества содержат на удивление длинные арифметические прогрессии. Этот факт был впервые замечен Ж. Бургейном (см. работу [67]). В последнее время появилось много работ по этой тематике (см., например, статьи [62], [133]-[138]). Мы не можем даже упомянуть все имеющиеся здесь результаты, а коснемся лишь некоторых из них. В работе [67] Ж. Бургейн получил следующий результат.

Теорема 34 (Бургейн). Пусть $A, B \subseteq[N]$ - некоторые множества, причем $|A|=\gamma N,|B|=\delta N$. Тогда существует абсолютная константа $c>0$ такая, что множество $A+B$ содержит арифметическую прогрессию длины не менъше, чем

$$
\exp \left(c\left((\gamma \delta \log N)^{1 / 3}-\log \log N\right)\right) .
$$

С другой стороны, И. Ружа нашел нижнюю оценку для длины максимальной арифметической прогрессии в множестве $A+A$ (см. [133]).

Теорема 35 (Ружа). Пусть $\varepsilon>0$ - произвольное число. Тогда найдется число $p_{0}(\varepsilon)$ такое, что для всех простых $p, p>p_{0}(\varepsilon)$, существует симметричное (т.е. $A=-A$ ) множество $A \subseteq \mathbb{Z}_{p},|A|>(1 / 2-\varepsilon) p$, такое, что $A+A$ не содержит арифметических прогрессий, длины которых больше, чем $\exp \left((\log p)^{2 / 3+\varepsilon}\right)$.

Так как для любого множества $A \subseteq \mathbb{Z}_{p},|A| \geqslant p / 2$, выполнено $A+A=\mathbb{Z}_{p}$ (см. раздел 8, рассуждения после формулы (71)), то константа $1 / 2$ в теореме 35 является неулучшаемой. Сравним теоремы 34 и 35. Предположим, что параметры $\gamma$ и $\delta$ в теореме 34 не зависят от $N$. Тогда, по этой теореме, для любого $A$ множество $A+A$ содержит арифметическую прогрессию длины не меньше, чем $\exp \left(c(\log N)^{1 / 3}\right)$, где $c$ - некоторая константа. С другой стороны, 
по теореме 35 найдется $A$ положительной плотности такое, что $A+A$ не содержит прогрессий длины больше, чем $\exp \left(c(\log N)^{2 / 3}\right)$. Как мы видим, оценки в теоремах Бургейна и Ружи достаточно близки.

В работе [134] Г. А. Фрейман, Х. Хальберстам и И. Ружа рассмотрели вопрос об арифметических прогрессиях в множествах вида $A+A+A$ и доказали следующий результат.

Теорема 36 (Фрейман, Хальберстам, Ружа). Пусть $N$ - натуральное число, $\delta>0$ u $A \subseteq \mathbb{Z}_{N}$ - произвольное множество мощности $\delta N$. Тогда множество $A+A+A$ содержит арифметическую прогрессию длины не менъше, чем $c \delta N^{C \delta^{3}}$, где $c, C>0$ - абсолютные константы.

В той же статье три названных автора доказали теорему, аналогичную теореме 35 , а именно, они построили множество $A \subseteq \mathbb{Z}_{N}$ такое, что $A+A+A$ не содержит арифметических прогрессий, длины которых больше, чем $2 N^{\log (1 / \delta)}$.

В работе [62] Грин улучшил обе теоремы 34 и 36.

ТеОрема 37 (Грин). Пусть $A, B \subseteq[N]$ - некоторые множества, причем $|A|=\alpha N,|B|=\beta N$. Тогда существует абсолютная константа $c>0$ такая, что множество $A+B$ содержит арифметическую прогрессию длины не меньше, чем $\exp \left(2^{-5}(\alpha \beta \log N)^{1 / 2}-c \log \log N\right)$.

ТеОрема 38 (Грин). Пусть $N$ - натуральное число, $\delta>0 u A \subseteq \mathbb{Z}_{N}$ - произвольное множество мощности $\delta N$. Тогда множество $A+A+A$ содержит арифметическую прогрессию длины не менъше, чем

$$
2^{-24} \delta^{5}\left(\log \left(\frac{1}{\delta}\right)\right)^{-2} N^{\delta^{2} /(250 \log (1 / \delta))} .
$$

Наконец, наилучший на сегодняшний день результат об арифметических прогрессиях в суммах не менее трех множеств принадлежит Т. Сандерсу (см. [139] и [140]).

Теорема 39 (Сандерс). Пусть $k$ - натуральное число, $k \geqslant 3$. Пусть также $A_{1}, \ldots, A_{k} \subseteq \mathbb{Z}_{N}$ - произвольные множества и $\delta$ - среднее геометрическое плотностей этих множеств. Тогда множество $A_{1}+\cdots+A_{k}$ содержит арифметическую прогрессию длины $c \delta^{C k^{3} \delta^{-1 /(k-2)}} N^{c k^{-2} \delta^{1 /(k-2)}}$, где $c, C>0$ некоторые абсолютные константы.

В этом разделе мы докажем теорему 37 и разберем простейший случай теоремы 39 в ситуации, когда $\mathbf{G}=\mathbb{Z}_{2}^{n}$ (см. теорему 41 ниже). Доказательство результата Сандерса проходит в духе рассуждений из раздела 3 . В теореме Грина используются абсолютно другие соображения, совершенно нетривиальным образом развивающие подход из [67]. Мы начнем доказательство теоремы 37 с формулировки одного более общего факта. Нам понадобится определение.

ОПРЕДЕЛЕНИЕ 5 . Пусть $S \subseteq \mathbb{Z}_{N}$ - некоторое множество и $\varepsilon \in(0,1)$ - па-

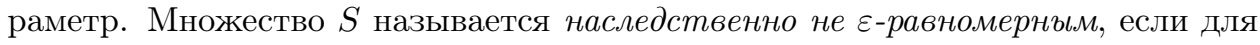
всякого $S^{\prime} \subseteq S$ выполнено

$$
\left|\widehat{S}^{\prime}(r)\right| \geqslant \varepsilon\left|S^{\prime}\right| \quad \forall r \neq 0 .
$$


Дополнения в группе $\mathbb{Z}_{N}$ до множеств, являющихся наследственно не $\varepsilon$-равномерными, обладают следующим свойством.

Теорема 40 (Грин). Пусть $\varepsilon \in(0,1)$ - действительное число такое, что $\varepsilon \geqslant 4000(\log \log N) /(\log N)^{1 / 2}, u S \subseteq \mathbb{Z}_{N}$ - наследственно не $\varepsilon$-равномерное множество. Тогда дополнение до $S$ содержит арифметическую прогрессию длины $e^{\varepsilon \sqrt{\log N} / 32}$.

Ясно, что из теоремы 40 вытекает теорема 37. Действительно, пусть $S-$ дополнение в $\mathbb{Z}_{N}$ до $A+B$. Пусть также $S^{\prime} \subseteq S$ - произвольное множество. Тогда, по определению, $\sum_{x} S^{\prime}(x)(A * B)(x)=0$. Перепишем последнее равенство, используя преобразование Фурье. Имеем

$$
0=\frac{1}{N} \sum_{r} \widehat{S}^{\prime}(r) \widehat{A}(r) \widehat{B}(r)=\frac{\left|S^{\prime}\right||A||B|}{N}+\frac{1}{N} \sum_{r \neq 0} \widehat{S}^{\prime}(r) \widehat{A}(r) \widehat{B}(r),
$$

откуда по неравенству Коши-Буняковского и равенству Парсеваля получаем

$$
|A|^{1 / 2}|B|^{1 / 2} N \max _{r \neq 0}\left|\widehat{S}^{\prime}(r)\right| \geqslant\left|S^{\prime}\right||A||B|,
$$

и мы видим, что множество $S$ является наследственно не $(\alpha \beta)^{1 / 2}$-равномерным. Если $(\alpha \beta)^{1 / 2} \geqslant 4000(\log \log N) /(\log N)^{1 / 2}$, то по теореме 40 дополнение до $S$, т. е. множество $A+B$, содержит арифметическую прогрессию длины $\exp \left(2^{-5}(\alpha \beta \log N)^{1 / 2}\right)$. Если же $(\alpha \beta)^{1 / 2} \leqslant 4000(\log \log N) /(\log N)^{1 / 2}$, то теорема 37 выполнена с константой $c=4000: 32=125$.

Итак, нам необходимо убедиться в справедливости теоремы 40. Пусть $\omega \in(0,1)$ - параметр и $\Omega \subseteq S, \Omega=\omega N,-$ некоторое множество, на котором достигается минимум выражения $\max _{r \neq 0}|\widehat{\Omega}(r)|$. Обозначим этот минимум через $\eta|\Omega|$. Поскольку $S$ не является наследственно $\varepsilon$-равномерным, то $\eta \geqslant \varepsilon$.

Основная идея доказательства состоит в том, что мы попытаемся модифицировать множество $\Omega$, т. е. превратить его в новое множество $\Omega^{\prime},\left|\Omega^{\prime}\right|=|\Omega|$, так, чтобы функционал $\max _{r \neq 0}\left|\widehat{\Omega^{\prime}}(r)\right|$ стал как можно меньше. Но поскольку строго меньше $\eta|\Omega|$ он стать не может, то это будет означать, что $\Omega$ (а точнее его дополнение) обладает некоторыми специфическими структурными свойствами. Отсюда уже недалеко до утверждения о специальной структуре дополнения множества $S$.

Модифицировать множество $\Omega$ мы будем так: удалим множество $X$ из $\Omega$, а затем добавим множество $D$ в $\Omega,|D|=|X|:=t$, где $t$ - параметр. При этом, конечно, может получится не множество, а мультимножество. С этой сложностью мы будем бороться в самом конце доказательства. Итак, метод Грина состоит из двух этапов - построения множества $D$ и множества $X$.

1. Построение множества $D$. Здесь мы будем использовать теорему 25 из раздела 6 (также можно применить теорему 31). Рассмотрим множество больших тригонометрических сумм

$$
\mathscr{R}=\left\{r:|\widehat{\Omega}(r)| \geqslant \eta \frac{|\Omega|}{2}\right\} .
$$


Лемма 15. Множество Бора $\mathscr{B}(\mathscr{R}, \eta / 64)$ содержит арифметическую прогрессию Р длинъ

$$
\frac{\eta^{3}}{2^{7} \log (1 / \omega)} N^{\eta^{2} /(2 \log (1 / \omega))}
$$

Действительно, по теореме 25 любой элемент множества $\mathscr{R}$ может быть представлен в виде комбинаций с коэффициентами \pm 1 элементов множества $\Lambda$. Пользуясь неравенством треугольника, получаем отсюда $\mathscr{B}(\Lambda, \eta /(64|\Lambda|)) \subseteq$ $\mathscr{B}(\mathscr{R}, \eta / 64)$, а дальше применяем лемму 2 к множеству $\mathscr{B}(\Lambda, \eta /(64|\Lambda|))$. Переход от множества $\mathscr{R}$ к меньшему множеству $\Lambda$ и составляет выигрыш от использования теоремы 25.

Пусть

$$
C:=\left\{x:|(P+x) \cap \Omega| \leqslant \frac{16 \omega}{\eta}|P|\right\} .
$$

Назовем такие точки группы $\mathbb{Z}_{N}$ хорошими. Поскольку

$$
|P||\Omega|=\sum_{x}|(P+x) \cap \Omega| \geqslant \sum_{x \notin C}|(P+x) \cap \Omega| \geqslant(N-|C|) \frac{16 \omega}{\eta}|P|,
$$

то хороших точек не меньше $(1-\eta / 16) N$. Другими словами, множество $C-$ это почти все $\mathbb{Z}_{N}$, и поэтому его коэффициенты Фурье малы. Действительно, пусть $C^{d}=\mathbb{Z}_{N} \backslash C$. Тогда для всех $r \neq 0$ выполнено

$$
|\widehat{C}(r)|=\left|\widehat{C^{d}}(r)\right| \leqslant\left|C^{d}\right| \leqslant \eta \frac{N}{16} \leqslant \eta \frac{|C|}{8},
$$

и мы можем, наконец, выбрать множество $D$. Нам понадобится вариант вероятностной леммы о больших уклонениях (см. [62]).

Лемма 16. Пусть $X_{j}, j \in[n]$, - независимые комплексные случайные величинь, $\left|X_{j}\right| \leqslant 1$, с нулевым математическим ожиданием и дисперсиями $\sigma_{j}^{2}$. Пусть также $t \geqslant 0$ - действительное число такое, что $\sigma^{2}:=\sum_{j} \sigma_{j}^{2} \geqslant 6$ nt.
Тогда

$$
\mathrm{P}\left(\left|\sum_{j} X_{j}\right| \geqslant n t\right) \leqslant 4 e^{-n^{2} t^{2} /\left(8 \sigma^{2}\right)} .
$$

Выберем множество $D \subseteq C$ случайным образом - элемент $x$ принадлежит множеству $D$ с вероятностью $p:=t /|C|$.

Лемма 17. Пусть $t \gg \eta^{-2} \log N$. Тогда найдется множество $D \subseteq C$ такое, чmo $|D|=t u \max _{r \neq 0}|\widehat{D}(r)| \leqslant \eta t / 4$.

Вычисления совершенно стандартные (см., например, [141]). Пусть множество $E \subseteq C$ выбирается случайным образом с вероятностью $p$. Тогда $r$-й коэффициент Фурье множества $E$ - это сумма независимых случайных величин $X_{j}^{(r)}(x)=E(x) e(-r x), \mathrm{E} \widehat{E}(r)=p \widehat{C}(r)$ и по лемме 16

$$
\mathrm{P}\left(|\widehat{E}(r)| \geqslant \frac{\eta t}{6}\right) \leqslant \mathrm{P}\left(|\widehat{E}(r)-\mathrm{E} \widehat{E}(r)| \geqslant \frac{\eta t}{24}\right) \leqslant 4 e^{-\eta^{2} t / 5000} .
$$


Аналогично

$$
\mathrm{P}\left(|| E|-t| \geqslant \frac{\eta t}{24}\right) \leqslant 4 e^{-\eta^{2} t / 5000} .
$$

По условию $t \gg \eta^{-2} \log N$, и, следовательно, с положительной вероятностью для всех $r \neq 0$ выполнено

$$
|\widehat{E}(r)| \leqslant \eta t / 6 \quad \text { и } \quad|| E|-t| \leqslant \eta t / 24 .
$$

Удаляя или добавляя не более чем $\eta t / 24$ точек в множество $D$, получаем утверждение леммы.

Опишем второй этап доказательства.

2. Построение множества $X$. Здесь используются совсем простые рассуждения. Выберем множество $X \subseteq \Omega$ случайным образом. Тогда аналогично лемме 17 доказывается следующее утверждение.

Лемма 18. Пусть $\beta N \geqslant t \gg \eta^{-2} \log N$. Тогда найдется множество $X \subseteq \Omega$, $|X|=t$, такое, что для всех $r \neq 0$ выполнено

$$
\left|\widehat{X}(r)-\frac{t}{|\Omega|} \widehat{\Omega}(r)\right| \leqslant \frac{\eta t}{12} .
$$

Положим $\Omega_{1}=(\Omega \backslash X) \cup D$. Этот объект представляет собой мультимножество. Тем не менее можно оценить его коэффициенты Фурье.

ЛЕмма 19. Имеем

$$
\max _{r \in \mathscr{R}, r \neq 0}\left|\widehat{\Omega}_{1}(r)\right| \leqslant \eta\left|\Omega_{1}\right|-\frac{\eta t}{6}
$$

и для всех $r \neq 0$ выполнено

$$
\left|\widehat{\Omega}_{1}(r)\right| \leqslant \frac{\eta\left|\Omega_{1}\right|}{2}+\frac{\eta t}{3} .
$$

ДокАЗАТЕЛЬСТво. Из построения $D$ и $X$, получаем

$$
\widehat{\Omega}_{1}(r)=\widehat{\Omega}(r)-\widehat{X}(r)+\widehat{D}(r)=\left(1-\frac{1}{|\Omega|}\right) \widehat{\Omega}(r)+Q,
$$

где $Q \leqslant \eta t / 3$. Если $r \in \mathscr{R}, r \neq 0$, то, вспоминая определение величины $\eta$ и учитывая неравенство $|\widehat{\Omega}(r)| \geqslant \eta|\Omega| / 2$, убеждаемся в справедливости (66). Если же $r \notin \mathscr{R}, r \neq 0$, то $|\widehat{\Omega}(r)| \leqslant \eta|\Omega| / 2$ и оценка (67) выполнена. Лемма 19 доказана.

Итак, мы построили мультимножество $\Omega_{1}$, у которого все коэффициенты Фурье меньше $\eta\left|\Omega_{1}\right|$. Если бы, во-первых, $\Omega_{1}$ было множеством и, во-вторых, выполнялось включение $\Omega_{1} \subseteq S$, то мы бы получили противоречие с экстремальными свойствами $\Omega$. Сейчас мы попробуем сдвинуть множество $D$ так, чтобы новое мультимножество $\Omega^{\prime}$ обладало этими двумя свойствами.

Пусть $D=\left\{d_{1}, \ldots, d_{t}\right\}$ и $D^{\prime}=\left\{d_{1}+x_{1}, \ldots, d_{t}+x_{t}\right\}$, где $x_{j}$ - элементы из прогрессии $P$. Положим $\Omega^{\prime}=(\Omega \backslash X) \cup D^{\prime}$. Докажем следующую лемму. 
Лемма 20. Пусть $t \leqslant \eta \omega N / 10$. Тогда для мультимножества $\Omega^{\prime}$ выполнено неравенство $\max _{r \neq 0}\left|\widehat{\Omega}^{\prime}(r)\right|<\eta\left|\Omega^{\prime}\right|$.

ДоказАтельство. Пусть $r \in \mathscr{R}$. Тогда из неравенства (67) и того факта, что вклад множества $D^{\prime}$ в коэффициенты Фурье $\Omega^{\prime}$ отличается от соответствующего вклада $D$ в $\Omega_{1}$ не более чем на $2 t$, получаем

$$
\left|\widehat{\Omega}^{\prime}(r)\right| \leqslant \frac{\eta\left|\Omega^{\prime}\right|}{2}+5 t<\eta\left|\Omega^{\prime}\right|
$$

Если же $r \in \mathscr{R}, r \neq 0$, то

$$
\left|\widehat{\Omega}^{\prime}(r)-\widehat{\Omega}_{1}(r)\right| \leqslant \sum_{j=1}^{t}\left|e\left(-r\left(d_{j}+x_{j}\right)\right)-e\left(-r x_{j}\right)\right| \leqslant t \max _{j}\left|e\left(-r x_{j}\right)-1\right| \leqslant \frac{\eta t}{8},
$$

поскольку $P \subseteq \mathscr{B}(\mathscr{R}, \eta / 64)$. Применяя (67), получаем утверждение леммы.

Приступим к непосредственному доказательству теоремы 40. Предположим, что для всех $j \in[t]$ выполнено $\left|\left(d_{j}+P\right) \cap(S \backslash \Omega)\right| \geqslant t$. Тогда возьмем $d_{1}$ и найдем $x_{1} \in P$ такой, что $d_{1}+x_{1} \in S \backslash \Omega$. Далее, берем $d_{2}$ и находим $x_{2} \in P$ такой, что $d_{1}+x_{1} \in(S \backslash \Omega) \backslash\left\{d_{1}+x_{1}\right\}$. И так далее. В конце концов мы найдем сдвиги всех элементов $d_{j}$, которые лежат в $S$, но не лежат в $\Omega$. Это позволит нам построить множество $\Omega^{\prime} \subseteq S$ с ненулевыми коэффициентами Фурье, меньшими, чем $\eta\left|\Omega^{\prime}\right|$. Но последнее неравенство невозможно из-за экстремальных свойств $\Omega$. Значит, найдется $d_{j} \in D$ такое, что $\left|\left(d_{j}+P\right) \cap(S \backslash \Omega)\right|<t$. Вспоминая, что точка $d_{j}-$ хорошая, получаем

$$
\left|S^{d} \cap\left(d_{j}+P\right)\right| \geqslant|P|-\frac{16 \omega}{\eta}|P|-t \geqslant\left(1-\frac{32 \omega}{\eta}\right)|P|,
$$

где параметр $t$ выбран так, что $t \leqslant 16 \omega|P| / \eta$, и $S^{d}=\mathbb{Z}_{N} \backslash S$. Пусть также $|P| \geqslant \eta /(32 \beta)$. Из оценки (68) и элементарных соображений среднего, легко видеть, что множество $S^{d}$ содержит арифметическую прогрессию длины $\eta /(32 \beta)$. Напомним, что ранее мы предполагали выполнение неравенств $t \gg \eta^{-2} \log N$ и $t \leqslant \eta \omega N / 10$. Кроме того, всегда $\eta \geqslant \varepsilon$. Выбирая параметры

$$
\omega=e^{-\eta \sqrt{\log N} / 16}, \quad t \sim \eta^{-2} \log N
$$

и производя небольшие вычисления, получаем теорему 40.

Докажем теперь специальный случай теоремы 39 (см. [140]). Пусть $\mathbf{G}=\mathbb{Z}_{2}^{n}$, $k=4$ и $A_{1}=A_{2}=A_{3}=A_{4}$. Мы рассматриваем эту ситуацию лишь из соображений простоты. В общем случае используются довольно похожие рассуждения (ср. теоремы 2 и 9 раздела 3 ).

Теорема 41 (Сандерс). Пусть $\mathbf{G}=\mathbb{Z}_{2}^{n} u A \subseteq \mathbf{G},|A|=\delta N$. Тогда $2 A-2 A$ содержит подпространство коразмерности $C \delta^{-1 / 2}$, где $C>0$ - некоторая абсолютная константа.

Читатель может заметить, что оценки теоремы 41 лучше, чем в теореме 39. Как и в теореме Рота, доказательство использует итерационную лемму. 
Лемма 21. Пусть $A \subseteq \mathbf{G},|A|=\delta N$ - некоторое множество. Тогда имеет место альтернатива:

1) либо $2 A-2 A=\mathbf{G}$;

2) либо существуют подпространство $V$ коразмерности 1 , множество $A^{\prime} \subseteq V$ u $x \in \mathbf{G}$ maкuе, чmo

(a) $A^{\prime}+x \subseteq A$,

(b) $\left|A^{\prime}\right| \geqslant\left(\delta+\delta^{3 / 2}\right)|V|$.

Действительно, если $2 A-2 A \neq \mathbf{G}$, то множество $\Omega:=\mathbf{G} \backslash(2 A-2 A)$ непусто. Ясно, что $\sum_{x} \Omega(x)(A * A * A * A)(x)=0$. Перепишем последнее равенство в терминах коэффициентов Фурье. Имеем

$$
0=\sum_{r} \widehat{\Omega}(r) \widehat{A}^{4}(r)=|\Omega||A|^{4}+\sum_{r \neq 0} \widehat{\Omega}(r) \widehat{A}^{4}(r) .
$$

Пусть $\alpha N:=\max _{r \neq 0}|\widehat{A}(r)|$. Используя равенство Парсеваля, находим

$$
|\Omega||A|^{4} \leqslant|\Omega||A| N(\alpha N)^{2},
$$

откуда $\alpha \geqslant \delta^{3 / 2}$. Значит, для некоторого $r \neq 0$ выполнено $|\widehat{A}(r)|=\alpha N \geqslant$ $\delta^{3 / 2} N$. Пусть $H_{0}=\{x \in \mathbf{G}:\langle x, r\rangle=0\}$ и $H_{1}=\{x \in \mathbf{G}:\langle x, r\rangle=1\}$. Тогда $\left|H_{0} \cap A\right|+\left|H_{1} \cap A\right|=|A|=\delta N$ и $\left|H_{0} \cap A\right|-\left|H_{1} \cap A\right|=\widehat{A}(r)$. Так как $|\widehat{A}(r)| \geqslant \delta^{3 / 2} N$, то либо $\left|H_{0} \cap A\right| \geqslant\left(\delta+\delta^{3 / 2}\right) N / 2$, либо $\left|H_{1} \cap A\right| \geqslant\left(\delta+\delta^{3 / 2}\right) N / 2$. Полагая $V=H_{0}$, $A^{\prime}=H_{0} \cap A, x=0$ в первом случае и $V=H_{0}, A^{\prime}=\left(H_{1} \cap A\right)-x, x \in H_{1}-$ любой элемент, во втором случае, получаем лемму 21.

ДокАЗАтЕЛЬСтво теОРемы 21. Применим лемму 21 к множеству $A$. Если выполнена первая альтернатива леммы, то доказывать нечего. Если же справедлива вторая альтернатива, то применим лемму к множеству $A^{\prime}$. И так далее. Поскольку переход от множества $A$ к множеству $A^{\prime}$ сопровождается увеличением плотности на величину $\delta^{3 / 2}$, то легко видеть, что наш итерационный процесс не может сделать более чем $C \delta^{-1 / 2}$ шагов (см. доказательство теоремы 9). Здесь $C>0$ - некоторая абсолютная константа. Поэтому итерационный процесс когда-нибудь остановится на шаге 1). Иными словами, некоторое подпространство $W$ коразмерности не больше, чем $C \delta^{-1 / 2}$, есть $2 \widetilde{A}-2 \widetilde{A}$ для некоторого множества $\widetilde{A}$. Легко видеть, что найдется трансляция множества $\widetilde{A}$, которая содержится в $A$, и, следовательно, $2 \widetilde{A}-2 \widetilde{A} \subseteq 2 A-2 A$. Поэтому множество $2 A-2 A$ содержит подпространство $W$ коразмерности не больше, чем $C \delta^{-1 / 2}$, и теорема доказана.

Методы гармонического анализа, рассмотренные в этом разделе, позволяют находить комбинаторные конфигурации лишь в суммах достаточно плотных множеств. Недавно появилась статья [142], где был, в частности, доказан следующий результат.

Теорема 42 (Крут-Ружа-Чоен). Пусть $k$ - нечетное число. Пусть также $A, B \subseteq[N]$ - некоторые множества $u|A||B| \geqslant 6 N^{2-2 /(k-1)}$. Тогда $A+B$ содержит арифметическую прогрессию длины $k$. 
Метод доказательства теоремы 42 совершенно элементарен и использует принцип Дирихле. Теорема 42 нетривиальна для достаточно “тощих" множеств, скажем, мощности $N^{1-\varepsilon}$, где $\varepsilon>0$ - некоторая константа. Тем не менее, для плотных множеств, как легко видеть, например, из теоремы 39, методы анализа Фурье дают лучшие оценки.

\section{8. Теорема Чеботарёва и принцип неопределенности}

Пусть $\mathbf{G}$ - конечная абелева группа, а $f$ - произвольная комплексная функция, заданная на этой группе. Обозначим через supp $f$ носитель функции $f$. Иными словами, supp $f=\{x \in \mathbf{G}: f(x) \neq 0\}$. Хорошо известно, что между мощностями множеств supp $f$ и supp $\hat{f}$ существует связь (см., например, [143] или [144]).

УтвеРЖДЕНИЕ 2 (принцип неопределенности). Пусть $f: \mathbf{G} \rightarrow \mathbb{C}-$ nроизвольная функиия, не равная тождественно нулю. Тогда

$$
|\operatorname{supp} f| \cdot|\operatorname{supp} \hat{f}| \geqslant|\mathbf{G}| \text {. }
$$

Действительно, пусть $\max _{\xi \in \widehat{G}}|\hat{f}(\xi)|$ достигается в точке $\xi_{0}$. Применяя неравенство Коши-Буняковского и равенство Парсеваля, находим

$$
\begin{aligned}
0 & <\left|\hat{f}\left(\xi_{0}\right)\right| \leqslant \sum_{x \in \operatorname{supp} f}|f(x)| \leqslant|\operatorname{supp} f|^{1 / 2}\left(\sum_{x \in \mathbf{G}}|f(x)|^{2}\right)^{1 / 2} \\
& =|\operatorname{supp} f|^{1 / 2}\left(|\mathbf{G}|^{-1} \sum_{\xi \in \operatorname{supp} \hat{f}}|\hat{f}(\xi)|^{2}\right)^{1 / 2} \leqslant|\operatorname{supp} f|^{1 / 2}|\mathbf{G}|^{-1 / 2}|\operatorname{supp} \hat{f}|^{1 / 2}\left|\hat{f}\left(\xi_{0}\right)\right|,
\end{aligned}
$$

откуда получаем требуемое неравенство.

Совсем недавно (см. [144]) обнаружилось, что в случае, когда $\mathbf{G}=\mathbb{Z} / p \mathbb{Z}$, где $p$ - простое, оценку (69) можно значительно улучшить.

ТеОРема 43 (Тао, принцип неопределенности в группе $\mathbb{Z} / p \mathbb{Z}$ ). Пусть $p-$ простое число и $f: \mathbb{Z} / p \mathbb{Z} \rightarrow \mathbb{C}$ - произвольная функиия, не равная тождественно нулю. Тогда

$$
|\operatorname{supp} f|+|\operatorname{supp} \hat{f}| \geqslant p+1 \text {. }
$$

Как мы сейчас убедимся, теорема Т. Тао выше есть не что иное, как переформулировка известной теоремы Чеботарёва о минорах матрицы Фурье.

Теорема 44 (Чеботарёв). Пусть $p$ - простое число. Тогда все минорь матрицы $\left(e^{2 \pi i m n / p}\right)_{n, m=1}^{p}-$ ненулевые.

Выведем из теоремы Чеботарёва теорему 43. Пусть $A$ и $B$ суть два произвольных непустых подмножества $\mathbb{Z}_{p}$ равной мощности. Тогда теорема Чеботарёва утверждает, что оператор $T_{A, B}: f \rightarrow \hat{f} B$, где $f$ принадлежит пространству функций с носителем в $A$, является обратимым. Предположим, что неравенство (70) неверно. Иными словами, пусть для некоторой функции $f$, не равной тождественно нулю, выполнено $|\operatorname{supp} f|+|\operatorname{supp} \hat{f}| \leqslant p$. Положим $A=\operatorname{supp} f$ 
и выберем $B$ так, что $B \subseteq\left(\mathbb{Z}_{p} \backslash \operatorname{supp} \hat{f}\right)$ и $|B|=|A|$. Но тогда мы получим противоречие с обратимостью оператора $T_{A, B}$ и теорема 43 доказана.

Пользуясь обратимостью оператора $T_{A, B}$, легко видеть (см., например, работу [144]), что теорема 43 является абсолютно точной в том смысле, что для любых двух непустых множеств $A$ и $B,|A|+|B| \geqslant p+1$, найдется функция $f$ с supp $f=A$ и supp $\hat{f}=B$.

Заметим, наконец, что неравенство (70) не имеет места в произвольной конечной абелевой группе. Например, в ситуации, когда $\mathbf{G}=\mathbb{Z}_{p}^{n}$, для характеристической функции $f$ подпространства $P$ размерности $k, k \leqslant n$, выполнено $|\operatorname{supp} f|=|P|,|\operatorname{supp} \hat{f}|=|\mathbf{G}| /|P|$ и оценка (70) неверна.

Легко видеть, что из теоремы 43 вытекает известное неравенство Коши о суммах множеств в $\mathbb{Z} / p \mathbb{Z}$ :

$$
|A+B| \geqslant \min \{p,|A|+|B|-1\} .
$$

Действительно, отметим сначала, что если $|A|+|B|>p$, то $A+B=\mathbb{Z}_{p}$ и поэтому оценка (71) выполнена (равенство $A+B=\mathbb{Z}_{p}$ вытекает из того факта, что для всякого $n \in \mathbb{Z}_{p}$ множества $n-A$ и $B$ обязательно пересекаются). Пусть теперь $|A|+|B| \leqslant p$. Тогда найдутся два множества $X$ и $Y,|X|=p+1-|A|$, $|Y|=p+1-|B|$, такие, что $X \cup Y=\mathbb{Z}_{p}$. Отсюда $|X \cap Y|=|X|+|Y|-p=$ $p+2-|A|-|B|$. В силу обратимости оператора $T_{A, B}$ найдутся функции $f$ и $g$, обладающие свойствами $\operatorname{supp} f=A, \operatorname{supp} \hat{f}=X$ и $\operatorname{supp} g=B, \operatorname{supp} \hat{g}=Y$. Рассмотрим новую функцию $F=f * g$. Тогда $\operatorname{supp} \widehat{F}=X \cap Y$ и supp $F=A+B$. По теореме 43, имеем

$$
|\operatorname{supp} F|+|\operatorname{supp} \widehat{F}|=|A+B|+p+2-|A|-|B| \geqslant p+1,
$$

и все доказано.

Отметим, что недавно в работе [145] ее авторы, используя метод Тао, уточнили неравенство (71).

Теорема 45 (Гуо-Сан). Пусть $p$ - простое число и $A, B, S$ - непустые подмножества $\mathbb{Z} / p \mathbb{Z}$. Тогда

$$
|\{a+b: a \in A, b \in B, a-b \notin S\}| \geqslant \min \{p,|A|+|B|-2|S|-1\} .
$$

Нам осталось доказать теорему Чеботарёва 44. Существует довольно много доказательств этого замечательного результата (см., например, [144], [146]). В своем изложении мы следуем статье [146]. Пусть $\omega=e^{2 \pi i / p}$ и $\pi=\omega-1$. Тогда для всех натуральных $r$ и $s$ выполнено

$$
\omega^{r s}=(1+\pi)^{r s}=\sum_{k=0}^{r s}\left(\begin{array}{c}
r s \\
k
\end{array}\right) \pi^{k} .
$$

Пусть также $M=\left|\omega^{r_{i}} s_{j}\right|_{i, j \in[l]}-$ произвольный минор матрицы $\Phi$ размера $l \times l$. Пользуясь полилинейностью определителя и тождеством (72), находим

$$
\operatorname{det} M=\sum_{k_{1} \geqslant 0, \ldots, k_{l} \geqslant 0} D_{k_{1}, \ldots, k_{l}} \pi^{k_{1}+\cdots+k_{l}},
$$


где $D_{k_{1}, \ldots, k_{l}}$ - определитель матрицы, составленной из столбцов

$$
\left(\left(\begin{array}{c}
r_{i} s_{1} \\
k_{1}
\end{array}\right), \ldots,\left(\begin{array}{c}
r_{i} s_{l} \\
k_{l}
\end{array}\right)\right)_{i \in[l]}
$$

Рассмотрим многочлен $p_{s, k}(x)=\left(\begin{array}{c}x s \\ k\end{array}\right)$ степени $k$. Тогда столбцы наших определителей суть значения многочленов $p_{s_{1}, k_{1}}, \ldots, p_{s_{l}, k_{l}}$ в точках $r_{1}, \ldots, r_{l}$. Зафиксируем натуральное $d<l$ и предположим, что в последовательности $k_{1}, \ldots, k_{l}$ найдется $d+1$ чисел $k_{j}$ меньших $d$. Поскольку размерность пространства многочленов степени $t$ равна $t+1$, то соответствующие многочлены линейно зависимы и число $D_{k_{1}, \ldots, k_{l}}$ равно нулю. Из наших соображений вытекает, что все коэффициенты в разложении (73) до $\pi^{a}, a=0+1+2+\cdots+(l-1)=l(l-1) / 2$, равны нулю. Пусть $\left\{k_{1}, \ldots, k_{l}\right\}=\{0,1,2, \ldots, l-1\}$. Вычислим коэффициент перед $\pi^{a}$, т. е. определитель $D_{k_{1}, \ldots, k_{l}}$. Обозначим это целое число через $C$. Если мы докажем, что $C$ взаимно просто с $p$, то будет легко увидеть, что оно не делится на $\pi$ в кольце $\mathbf{Q}[\omega]$ и, следовательно, $\operatorname{det} M \neq 0$. Разлагая каждый из многочленов $p_{s, k}(x)$ по формуле $p_{s, k}(x)=s x(s x-1) \cdots(s x-k+1) / k !=s^{k} x^{k} / k !+g_{s, k}(x)$, где $g_{s, k}(x)$ - многочлен степени $k-1$, и используя рассуждения выше, находим

$$
C=\sum_{\sigma} \operatorname{sgn}(\sigma) \frac{s_{1}^{\sigma(0)} \cdots s_{l}^{\sigma(l-1)}}{0 ! 1 ! \cdots(l-1) !} \prod_{i, j \in[l]}\left(r_{j}-r_{i}\right) .
$$

При выводе последней формулы мы применили выражение для определителя Вандермонда. Воспользовавшись этим выражением еще раз, получаем

$$
C=\frac{1}{0 ! 1 ! \cdots(l-1) !} \prod_{i, j \in[l]}\left(r_{j}-r_{i}\right) \prod_{i, j \in[l]}\left(s_{j}-s_{i}\right) \neq 0 \quad(\bmod p),
$$

что и требовалось.

\section{9. Связь с аналитической теорией чисел}

Пусть $R \subseteq \mathbb{Z}_{p}^{*}-$ произвольная мультипликативная подгруппа. Вопрос об оценках тригонометрических сумм по подгруппам является старой задачей аналитической теории чисел (см., например, [47] или [147]). Иными словами, мы интересуемся оценкой сверху для величины

$$
S(R)=\max _{a \in \mathbb{Z}_{p}^{*}}\left|\sum_{x \in R} e(a x)\right| .
$$

Классическая оценка для $S(R)$ есть $S(R) \leqslant \sqrt{p}$ (см. [147]). Ясно, что для подгрупп порядка меньше, чем $\sqrt{p}$, эта оценка тривиальна. В работах [48], [148], [149] были получены неравенства вида

$$
S(R) \leqslant|R| p^{-\varepsilon}
$$

где $|R| \geqslant p^{c}, c>0$ - некоторая константа и $\varepsilon=\varepsilon(c)>0$. Так, в работе [48] было показано, что оценка (74) выполнена, если $c>1 / 4-$ произвольное. До последнего времени это был наименьший размер подгруппы, начиная с которого 
имелась нетривиальная оценка (74). В статье [16] (см. также [15]) неравенство (74) было доказано для любой положительной константы $c$.

Теорема 46 (Бургейн-Конягин). Пусть $\delta>0$ - действительное число. Тогда найдутся натуральное число $p_{0}(\delta)$ и $\varepsilon(\delta) \in(0,1)$ такие, что для всех простых $p, p \geqslant p_{0}$, любой мультипликативной подгруппы $R \subseteq \mathbb{Z}_{p}^{*},|R| \geqslant p^{\delta}$, выполнено

$$
S(R) \leqslant|R| p^{-\varepsilon}
$$

Ограничение $|R| \geqslant p^{\delta}$ в теореме 46 было затем ослаблено в статье [21].

Поскольку любая подгруппа $R$ в $\mathbb{Z}_{p}^{*}$ имеет вид $R=\left\{x^{k}: x \in \mathbb{Z}_{p}\right\}$ для некоторого натурального $k$ и справедливо равенство

$$
|R|=(p-1) /(k, p-1),
$$

то теорему 46 можно переформулировать следующим образом.

Теорема 47 (Бургейн). Пусть $\delta$ - действительное число, $p$ - достаточно большое простое, $k$ - натуральное число, $k<p-1 u(k, p-1)<p^{1-\delta}$. Тогда найдется $\varepsilon=\varepsilon(\delta)>0$ такое, что

$$
\left|\sum_{x=1}^{p-1} e\left(a x^{k}\right)\right|<p^{1-\varepsilon} .
$$

В работе [17] (см. также [15]) Бургейн получил аналогичные оценки для сумм

$$
\sum_{s=1}^{t_{1}} e\left(a \theta^{s}\right) \quad \text { и } \sum_{s, s^{\prime}=1}^{t_{1}} e\left(a \theta^{s}+b \theta^{s s^{\prime}}\right),
$$

где $a, \theta \neq 0, \theta$ - элемент порядка $t$ и $t \geqslant t_{1}>p^{\delta}$.

Теорема 47 была обобщена в статье [18].

Теорема 48 (Бургейн). Пусть $\delta$ - действительное число, $p$ - достаточно большое простое, $k_{i}$ - натуральные числа, $i \in[r], k_{i}<p-1 u$

$$
\left(k_{i}, p-1\right)<p^{1-\delta}, \quad i \in[r],
$$

a такље

$$
\left(k_{i}-k_{j}, p-1\right)<p^{1-\delta}, \quad i, j \in[r], \quad i \neq j .
$$

Пусть

$$
f(x)=\sum_{i=1}^{r} a_{i} x_{i}^{k}, \quad\left(a_{i}, p\right)=1 .
$$

Тогда найдется $\varepsilon=\varepsilon(\delta, r)>0$ maкое, что

$$
\left|\sum_{x=1}^{p-1} e(f(x))\right|<p^{1-\varepsilon} .
$$


Тригонометрические суммы (80) называются суммами Морделла. Более ранние результаты по суммам Морделла см. в [150], [151]. Теорема 48 была уточнена в статье [152]. Заметим, что условие (78) является необходимым. Действительно (см. [151]), пусть, например, $r=2, k_{1}=1, k_{2}=(p-1) / 2+1$ и $f(x)=x-x^{(p-1) / 2+1}$. Тогда из неравенства Вейля (см., например, [153]) легко вытекает, что

$$
\sum_{x=1}^{p-1} e(f(x))=\frac{p-1}{2}+\sum_{x^{(p-1) / 2}=-1} e(2 x)+e(1)=\frac{p-1}{2}+O(\sqrt{p}),
$$

поскольку $x^{(p-1) / 2}-$ это символ Лежандра числа $x$.

Полиномы вида (79) называют малочленами. В работе [154] был дан в некотором смысле окончательный результат, описывающий все малочлены, для которых возможны нетривиальные оценки тригонометрических сумм, аналогичные (80). Нам потребуется несколько определений. Напомним, что отклонением последовательности точек $x_{1}, \ldots, x_{n} \in[0,1)$ называется величина

$$
D_{n}=D_{n}\left(x_{1}, \ldots, x_{n}\right)=\sup _{0 \leqslant \alpha<\beta \leqslant 1}\left|\frac{\left|\left\{i: x_{i} \in[\alpha, \beta)\right\}\right|}{n}-(\beta-\alpha)\right| .
$$

Пусть $f: \mathbb{Z}_{p} \rightarrow \mathbb{Z}_{p}$ - произвольная функция. Обозначим отклонение последовательности $\{f(i) / p\}$ через $D_{p}(f)$. Здесь $\mathbb{Z}_{p}$ отождествляется с последовательностью $\{0,1, \ldots, p-1\}$. Наконец, если $p_{j}$ - последовательность простых чисел, то последовательность многочленов $f_{j}: \mathbb{Z}_{p_{j}} \rightarrow \mathbb{Z}_{p_{j}}$ называется хорошо распределенной, если для некоторого $\varepsilon>0$ имеем $D_{p_{j}}\left(f_{j}\right) \leqslant p_{j}^{-\varepsilon}$. Хорошо известно (см., например, [153]), что из нетривиальной оценки тригонометрических сумм по точкам последовательности вытекает, что последовательность хорошо распределена. С другой стороны, для многочлена $f(x)=a x^{k}, a \neq 0$, и произвольного простого $p$ имеем $D_{p}(f) \geqslant k / p$, поскольку, как легко видеть, полином $f(x)$ принимает одно и то же значение $\operatorname{deg} f=k$ раз. Поэтому мы можем переформулировать теоремы 46, 47 в такой форме.

ПредЛожениЕ 5. Последователъность ненулевых одночленов $f_{j}=a_{j} x^{k_{j}}$ хорошо распределена тогда и толъко тогда, когда для некоторого $\eta>0$ въполнено $\left(k_{j}, p_{j}-1\right)<p_{j}^{1-\eta}$, где $j$ - любое.

С помощью теоремы 48 С. В. Конягин получил аналогичный критерий для произвольных малочленов. Мы сформулируем лишь один результат из [154].

ТЕОРемА 49 (Конягин). Пусть $r$ - натуральное число $и f_{p_{j}} \in \mathbb{Z}_{p_{j}}$ - последовательность многочленов таких, что

$$
f_{j}(x)=\sum_{k_{j} \in K_{j}} a\left(k_{j}\right) x^{k_{j}},
$$

где $K_{j} \subseteq \mathbb{Z}_{+} u\left|K_{j}\right| \leqslant r$. Последовательность $\left\{f_{j}\right\}$ хорошо распределена тогда и только тогда, когда найдется $\eta>0$ такое, что ни одно из множеств $K_{j}$ не представляется в виде дизгюнктного оббединения множеств $K_{0}^{j}, \ldots, K_{s_{j}}^{j}$, обладающих следующими свойствами:

(a) $d_{j} \mid k$ для всех $k \in K_{0}^{j}$;

(b) $d_{j} \mid\left(k-k^{\prime}\right)$ для любого $i=1, \ldots, s_{j}$ и всех $k, k^{\prime} \in K_{i}^{j}$; 
(с) найдется $x_{0}^{j} \in \mathbb{Z}_{p_{j}}$ такой, что для любого $i=1, \ldots, s_{j}$ выполнено

$$
\sum_{k \in K_{i}^{j}} a(k)\left(x_{0}^{j}\right)^{k}=0
$$

где $d_{j}>p_{j}^{1-\eta} u d_{j} \mid\left(p_{j}-1\right)$.

Обобщение теоремы 48 на более широкий класс многочленов (представимых, грубо говоря, в виде суммы малочлена и многочлена степени $p^{1 / 2-\delta}, \delta>0$ ) см. в [22].

Основополагающая теорема 46 вытекает из результата Бургейна-КатцаТао [10] о суммах произведений в группе $\mathbb{Z}_{p}$. Независимое доказательство теоремы 46 см. в замечательной статье [155]. Более ранние работы по теории сумм произведений в упорядоченных группах, таких как $\mathbb{R}$ или $\mathbb{Z}$, см. в [156]-[159].

Теорема 50 (Бургейн-Катц-Тао). Пусть $\delta>0$ - действительное число. Тогда найдутся натуральное число $p_{0}(\delta)$ и вещественное $\varepsilon(\delta) \in(0,1)$ такие, что для всех простых $p, p \geqslant p_{0}$, и любого множества $A \subseteq \mathbb{Z}_{p},|A| \geqslant p^{\delta}$, выполнено

$$
\max \{|A+A|,|A \cdot A|\} \geqslant|A| p^{\varepsilon} .
$$

Теорема 50 (см. также ее усиления в [21]) говорит о том, что в $\mathbb{Z}_{p}$ не может быть множеств, имеющих одновременно маленькую сумму и произведение.

Помимо теорем 46, 47 еще одним следствием теоремы 50 является аналог теоремы Семереди-Троттера в группе $\mathbb{Z}_{p}$ (см. [10]). Пусть $p-$ простое число. Пусть также буквы $\mathscr{P}, \mathscr{L} \subseteq \mathbb{Z}_{p} \times \mathbb{Z}_{p}$ обозначают произвольные семейства точек и прямых.

Теорема 51 (Бургейн-Катц-Тао). Пусть $0<\beta<2$ - действительное число. Тогда для всех достаточно больших простых $р$ и произвольных множеств $\mathscr{P}$ и $\mathscr{L}$ maких, что

$$
|\mathscr{P}|,|\mathscr{L}|<p^{\beta}
$$

найдется $\varepsilon(\beta) \in(0,1)$, для которого выполнено

$$
|\{(P, L) \in \mathscr{P} \times \mathscr{L}: P \in L\}| \ll p^{(3 / 2-\varepsilon) \beta} .
$$

Оценка $p^{3 \beta / 2}$ в неравенстве (82) легко следует из неравенства Коши-Буняковского, поэтому величина $p^{-\varepsilon \beta}$ представляет собой нетривиальный понижающий множитель.

В статье [49] Бургейн, опираясь на теорему 51 и используя технику из [10], [15], [16], получил целый ряд новых результатов об оценках тригонометрических сумм. Мы отметим лишь некоторые из этих сумм:

$$
\max _{a \neq 0}\left|\sum_{x \in A} \sum_{y \in B} e\left(a\left(x y+x^{2} y^{2}\right)\right)\right|, \quad \max _{a \neq 0}\left|\sum_{x \in A} \sum_{y \in B} e\left(a\left(x y+g^{x+y}\right)\right)\right|,
$$

где $A, B$ - произвольные множества, $|A|,|B| \gg \sqrt{p}$, а $g$ - первообразный корень. Также (см. [20] и [21])

$$
\left|\sum_{x_{1} \in A_{1}, \ldots, x_{r} \in A_{r}} e\left(x_{1} \cdots x_{r}\right)\right| \leqslant\left|A_{1}\right| \cdots\left|A_{r}\right| p^{-\varepsilon}
$$


где $r \geqslant 2$ - натуральное число, $\varepsilon=\varepsilon(\delta)>0, A_{1}, \ldots, A_{r}$ - произвольные множества, $\left|A_{j}\right| \geqslant p^{\delta}, j \in[r]$, и $\prod_{j=1}^{r}\left|A_{j}\right| \geqslant p^{1+\delta}$. Ранее оценка (83) была известна только в случае $r=2$ (см., например, [153]). Кроме того, Бургейн доказал несколько новых интересных утверждений, иллюстрирующих феномен сумм произведений. Например, если $A \subseteq \mathbb{Z}_{p}-$ произвольное множество, $p^{\delta}<|A|<p^{1-\delta}$, и для некоторого $\varepsilon=\varepsilon(\delta)$ (это $\varepsilon$ может быть явно указано) выполнено $|A \cdot A|<p^{\varepsilon / 2}|A|$, то для всех $a \neq 0$ имеем

$$
|A \cap(A+a)|<p^{-\varepsilon / 3}|A|
$$

Наоборот, если $|A|<p^{1-\delta}$ и $|A+A|<|A|^{1+\varepsilon / 2}, \varepsilon=\varepsilon(\delta)$, то для всех $a \in \mathbb{Z}_{p}$ выполнено

$$
\left|A \cap a A^{-1}\right|<|A|^{1-\varepsilon / 4} .
$$

Приложения результатов статьи [49] к задачам теоретической компьютерной математики см. в [160]. Новые неравенства для тригонометрических сумм по подгруппам в группах $\mathbb{Z}_{q}$, где $q$ - составное, были получены в статьях [19], [23]. Применение комбинаторных методов для оценки тригонометрических с мультипликативными характерами см. в [161].

Итак, мы видели, что методы аддитивной комбинаторики позволяют доказывать новые теоретико-числовые утверждения. С другой стороны, как недавно выяснилось (см. [51], а также [162]), классические оценки тригонометрических сумм по аддитивным характерам дают новые результаты о суммах произведений. Приведем одну теорему из [51].

Теорема 52 (Гараев). Пусть $р$ - простое число $и A \subseteq \mathbb{Z}_{p}$ - произвольное множество. Тогда

$$
|A+A||A \cdot A| \gg \min \left\{p|A|, \frac{|A|^{4}}{p}\right\} \text {. }
$$

Доказательство. Можно считать, что $0 \notin A$. Рассмотрим уравнение

$$
x y^{-1}+z=w, \quad \text { где } x \in A \cdot A, y \in A, z \in A, w \in A+A .
$$

Ясно, что у этого уравнения есть $|A|^{3}$ решений вида $x=a_{1} a_{2}, y=a_{2}^{-1}, z=a_{3}$, $w=a_{1}+a_{3}$, где все $a_{i}$ принадлежат $A$, причем разным тройкам $\left(a_{1}, a_{2}, a_{3}\right)$ соответствуют различные решения $x, y, z, w$ уравнения (85). Пусть $J$ - число решений уравнения (85). Как мы только что доказали, $J \geqslant|A|^{3}$. С другой стороны,

$$
\begin{aligned}
|A|^{3} & \leqslant J=\frac{1}{p} \sum_{r \in \mathbb{Z}_{p}} \sum_{x \in A \cdot A} \sum_{y \in A} \sum_{z \in A} \sum_{w \in A+A} e\left(r\left(x y^{-1}+z-w\right)\right) \\
& \leqslant \frac{|A|^{2}|A+A||A \cdot A|}{p}+\frac{1}{p} \sum_{r \in \mathbb{Z}_{p}^{*}}|\widehat{A}(-r)|\left|(A+A)^{\wedge}(r)\right|\left|\sum_{x \in A \cdot A} \sum_{y \in A^{-1}} e(r x y)\right| .
\end{aligned}
$$


Применяя оценку тригонометрической суммы по произведениям множеств (см., например, [153]), получаем

$$
\left|\sum_{x \in A \cdot A} \sum_{y \in A^{-1}} e(r x y)\right| \leqslant \sqrt{p|A \cdot A||A|}, \quad r \neq 0 .
$$

Отсюда и из неравенства Коши-Буняковского имеем

$$
|A|^{3} \leqslant J \leqslant \frac{|A|^{2}|A+A||A \cdot A|}{p}+\sqrt{p|A \cdot A||A|^{2}|A+A|},
$$

и теорема доказана.

Например, если $|A| \gg p^{2 / 3}$, то из оценки (84) вытекает неравенство

$$
\max \{|A+A|,|A \cdot A|\} \gg p^{1 / 2}|A|^{1 / 2} .
$$

Заметим, что последнюю оценку улучшить нельзя (см., например, [51]) и, следовательно, теорема 52 точна для множеств $A$ мощности $\gg p^{2 / 3}$. Оценка снизу на мощность $A$ возникла здесь не случайно, поскольку, как известно, аналитические методы хорошо работают только для достаточно больших множеств.

В доказательстве теоремы 52 была, фактически, получена асимптотическая формула для числа решений уравнения (85). Другие уравнения с переменными, принадлежащими произвольным множествам из $\mathbb{Z}_{p}$, рассматривались в работах [53]-[56]. Сформулируем только два результата.

Tеорема 53 [55]. Пусть $A, B, C, D \subseteq \mathbb{Z}_{p}$ - произвольные множества $и$ $\lambda \in \mathbb{Z}_{p}^{*}$ - любое. Тогда для числа решений $N_{1}(A, B, C, D)$ уравнения

$$
a b+c d=\lambda, \quad \text { где } a \in A, b \in B, c \in C, d \in D,
$$

справедлива асимптотическая формула

$$
N_{1}(A, B, C, D)=\frac{|A||B||C||D|}{p-1}+O\left((p|A||B||C||D|)^{1 / 2}\right) .
$$

Tеорема 54 [54]. Пусть $A, B, C, D \subseteq \mathbb{Z}_{p}$ - произволъные множества. Тогда для числа решений $N_{2}(A, B, C, D)$ уравнения

$$
a+b=c d, \quad \text { де } a \in A, b \in B, \quad c \in C, \quad d \in D,
$$

справедлива асимптотическая формула

$$
N_{2}(A, B, C, D)=\frac{|A||B||C||D|}{p-1}+O\left((p|A||B||C||D|)^{1 / 2}\right) .
$$

Метод доказательства теорем 53, 54 также состоит в использовании классических оценок тригонометрических сумм. В этих теоремах устанавливается разрешимость некоторых уравнений от четырех неизвестных, пробегающих произвольные достаточно плотные множества $A, B, C, D$. Например, если $|A|,|B|,|C|,|D| \gg p^{1-\varepsilon_{0}}$, где $\varepsilon_{0}>0$ - достаточно малое число, то уравнения (86), (87) разрешимы. Недавно автор (см. [116]) привел пример уравнения от трех переменных вида $f(x, y)=z$, где $f(x, y)$ - некоторый многочлен, также являющегося разрешимым для любых достаточно плотных множеств. Ясно, что уравнения от двух переменных с подобным свойством не существует. 


\section{0. Заключение}

Мы заканчиваем настоящий обзор формулировкой нескольких нерешенных проблем гармонического анализа, связанных с задачами комбинаторной теории чисел. Часть из этих проблем уже была сформулирована в предыдущих разделах, например, полиномиальная гипотеза Фреймана-Ружи (см. раздел 4).

Наиболее сложной нерешенной задачей остается гипотеза Эрдёша-Турана 1. Как было показано в разделе 3 , эта гипотеза тесно связана с проблемой изучения поведения функции $a_{k}(N)$. Заметим, что даже в простейшем случае $k=3$ точный порядок роста $a_{3}(N)$ до сих пор остается неизвестным. Недавно Грин и Тао анонсировали результат о верхней оценке величины $a_{k}(N), k \geqslant 4$, вида $a_{k}(N) \ll 1 /(\log N)^{C_{k}},\left(C_{k}>0\right.$ - абсолютная константа) и доказали аналогичный результат для функции $a_{4}(N)$ в группах $\mathbb{Z}_{p}^{n}$, где $p$ - простое, $p \neq 2,3$ (см. [46]). Относительно поведения величины $a_{3}(N)$ в группе $\mathbb{Z}_{3}^{n}$ Б. Грин высказал следующую гипотезу (см. [3]).

ГиПотеЗА 4 (Грин). Пусть $\mathbf{G}=\mathbb{Z}_{3}^{n}$. Тогда $a_{3}(N) \leqslant N^{-\varepsilon_{0}}$, где $\varepsilon_{0}>0-$ некоторая абсолютная постоянная.

Мы сформулируем одну, может быть чрезмерно смелую, гипотезу, из справедливости которой для множеств мощности порядка $N /(\log N)^{c}, c>1$, вытекает положительное решение проблемы Эрдёша-Турана в случае $k=3$, а также гипотеза 4 (если мощность множества оценивается снизу как $N^{1-c^{\prime}}, c^{\prime}>0$ ).

ГиПотезА 5. Пусть $A \subseteq \mathbb{Z}_{N}$ или $A \subseteq \mathbb{Z}_{p}^{n}, p \geqslant 3$, - произвольное множество без арифметических прогрессий длинъ три, некоторой достаточно большой мощности (например, $\left.N /(\log N)^{c}, c>1\right)$. Тогда найдется $r \neq 0$ такое, что $|\widehat{A}(r)| \geqslant \rho|A|$, где $\rho>0$ - абсолютная постоянная.

Справедливость гипотезы 5 для множеств положительной плотности легко вытекает из рассуждений раздела 3. Заметим также, что гипотеза 5 неверна для множеств без решений произвольного неаффинного линейного уравнения, скажем, $x+y=z$ (см. пример множества из теоремы 2.11 статьи [128]). Наконец, легко видеть, что для очень маленьких множеств мощности, скажем, $N^{1 / 3}$ гипотеза 5 также несправедлива. Например, можно взять случайное множество мощности $N^{1 / 3}$. Автор благодарен С. В. Конягину за это замечание.

В связи с полиномиальной гипотезой Фреймана-Ружи 3 (с. 146) возникает такой вопрос. Пусть $p$ - простое число и $S \subseteq \mathbb{Z}_{p}^{n}-$ некоторое множество, $|S|=\delta N$. Какова коразмерность подпространства, содержащегося в $2 A-2 A$ ? На данный момент наилучший результат здесь принадлежит Т. Сандерсу [140], а именно, эта коразмерность $c$ есть $O\left(\delta^{-1 / 2}\right)$ (см. раздел 7). Легко проверить, что из оценки $c \ll \log (1 / \delta)$ вытекает гипотеза Фреймана-Ружи.

Сам по себе интересен вопрос о строении множеств больших тригонометрических сумм. Каковы необходимые и достаточные условия для того, чтобы некоторое множество было множеством вида $\mathscr{R}_{\alpha}$ ? Неизвестен ответ даже на более специальный вопрос: что можно сказать о структуре множеств $\mathscr{R}_{\alpha}$, мощность которых близка к числу $\delta / \alpha^{2}-$ верхней границе для мощности множества больших тригонометрических сумм, вытекающей из равенства Парсе- 
валя? Подобные результаты могут помочь в решении задач комбинаторной теории чисел, использующих анализ Фурье.

До сих пор ни для одного линейного уравнения $c_{1} x_{1}+\cdots+c_{n} x_{n}=0$ при $n$, не зависящем от размера группы, не найдено точное значение его рамсеевского числа (см. раздел 5). Очень интересно иметь пример такого уравнения, для которого был бы известен хотя бы порядок поведения величины $R(\mathbf{c})$.

В заключение отметим, что остается открытой проблема, возможно центральная в аддитивной комбинаторике, об определении необходимых и достаточных условий для того, чтобы множество $S$ имело вид $A+A$. Автор надеется, что методы анализа Фурье могут быть полезны при ответе на этот вопрос.

Автор выражает благодарность С. В. Конягину и К. С. Рютину за ряд полезных обсуждений и замечаний.

\section{Список литературы}

[1] M. B. Nathanson, "Additive number theory. Inverse problems and the geometry of sumsets", Grad. Texts in Math., 165, Springer-Verlag, New York, 1996, ISBN: 0-387-94655-1, 293 pp.

[2] T. Tao, Van Vu, Additive combinatorics, Cambridge Stud. Adv. Math., Cambridge Univ. Press, Cambridge, 2006, ISBN: 978-0-521-85386-6, 512 pp.

[3] B. Green, "Finite field models in additive combinatorics", Surveys in combinatorics, London Math. Soc. Lecture Note Ser., 327, Cambridge, Cambridge Univ. Press, 2005, 1-27.

[4] И. Д. Шкредов, "Теорема Семереди и задачи об арифметических прогрессиях", УМH, 61:6 (2006), 111-178; англ. пер.: I. D. Shkredov, "Szemerédi's theorem and problems on arithmetic progressions", Russian Math. Surveys, 61:6 (2006), $1101-1166$.

[5] A. L. Cauchy, "Recherches sur les nombres", J. École Polytech., 9 (1813), 99-116.

[6] L. G. Shnirel'mann, "Über additive Eigenschaften von Zahlen", Math. Ann., 107:1 (1933), 649-690.

[7] W.T. Gowers, "A new proof of Szemerédi's theorem for arithmetic progressions of length four", Geom. Funct. Anal., 8:3 (1998), 529-551.

[8] W. T. Gowers, "A new proof of Szemerédi's theorem", Geom. Funct. Anal., 11:3 (2001), 465-588.

[9] B. Green, T. Tao, "An inverse theorem for the Gowers $U^{3}(G)$ norm, with applications", Proc. Edinb. Math. Soc. (2), 51:1 (2008), 73-153.

[10] J. Bourgain, N. Katz, T. Tao, "A sum-product estimate in finite fields and their applications", Geom. Funct. Anal., 14:1 (2004), 27-57.

[11] J. Solymosi, "On the number of sums and products", Bull. London Math. Soc., 37:4 (2005), 491-494.

[12] A. A. Glibichuk, S. V. Konyagin, "Additive properties of product sets in fields of prime order", Additive combinatorics, CRM Proc. Lecture Notes, 43, Amer. Math. Soc., Providence, RI, 2007, 279-286; arXiv: math/0702729.

[13] А. А. Глибичук, "Комбинаторные свойства множеств вычетов по простому модулю и задача Эрдёша-Грэхэма", Матем. заметки, 79:3 (2006), 384-395; англ. пер.: A. A. Glibichuk, "Combinational properties of sets of residues modulo a prime and the Erdös-Graham problem", Math. Notes, 79:3-4 (2006), 356-365.

[14] J. Bourgain, The sum-product theorem in $\mathbb{Z}_{q}$ with $q$ arbitrary, Preprint. 
[15] J. Bourgain, A. Glibichuk, S. Konyagin, "Estimate for the number of sums and products and for exponential sums in fields of prime order", J. London Math. Soc. (2), 73:2 (2006), 380-398.

[16] J. Bourgain, S. V. Konyagin, "Estimates for the number of sums and products and for exponential sums over subgroups in fields of prime order", C. R. Math. Acad. Sci. Paris, 337:2 (2003), 75-80.

[17] J. Bourgain, "Estimates on exponential sums related to the Diffie-Hellman distributions", Geom. Funct. Anal., 15:1 (2005), 1-34.

[18] J. Bourgain, "Mordell's exponential sum estimate revisited", J. Amer. Math. Soc., 18:2 (2005), 477-499.

[19] J. Bourgain, "Exponential sum estimates over subgroups of $\mathbb{Z}_{q}^{*}, q$ arbitrary", J. Anal. Math., 97 (2005), 317-355.

[20] J. Bourgain, Multilinear exponential sums in prime fields, Preprint.

[21] J. Bourgain, "Multilinear exponential sums in prime fields under optimal entropy condition on the sources", Geom. Funct. Anal., 18:5 (2009), 1477-1502.

[22] J. Bourgain, "Some arithmetical applications of the sum-product theorems in the finite fields", Geometric aspects of functional analysis, Lecture Notes in Math., Springer, Berlin, 2007, 99-116.

[23] J. Bourgain, M.-C. Chang, "Exponential sums estimates over subgroups and almost subgroups of $\mathbb{Z}_{q}^{*}$, where $q$ is composite with few prime factors", Geom. Funct. Anal., 16:2 (2006), 327-366.

[24] T. Tao, A quantitative ergodic theory proof of Szemerédi's theorem, arXiv: math. CO/0405251.

[25] T. Tao, "Norm convergence of multiple ergodic averages for commuting transformations", Ergodic Theory Dynam. Systems, 28:2 (2008), 657-688.

[26] V. Bergelson, A. Leibman, "Polynomial extentions of van der Waerden's and Szemerédi's theorems", J. Amer. Math. Soc., 9:3 (1996), 725-753.

[27] V. Bergelson, A. Leibman, "Set-polynomials and polynomial extension of the HalesJewett theorem", Ann. of Math. (2), 150:1 (1999), 33-75; arXiv: math/9907201.

[28] A. Leibman, "Pointwise convergence of ergodic averages for polynomial sequences of translations on a nilmanifold", Ergodic Theory Dynam. Systems, 25:1 (2005), 201-213.

[29] H. Furstenberg, Y. Katznelson, "A density version of the Hales-Jewett theorem", J. Anal. Math., 57 (1991), 64-119.

[30] A. W. Hales, A. I. Jewett, "Regularity and positional games", Trans. Amer. Math. Soc., 106:2 (1963), 222-229.

[31] B. Host, B. R. Kra, "Nonconventional ergodic averages and nilmanifolds", Ann. of Math. (2), 161:1 (2005), 397-488.

[32] B. Host, B. Kra, "Convergence of polynomial ergodic averages. Probability in mathematics", Israel J. Math., 149:1 (2005), 1-19.

[33] T. Ziegler, "A non-conventional ergodic theorem for a nilsystem", Ergodic Theory Dynam. Systems, 25:4 (2005), 1357-1370.

[34] T. Ziegler, "Universal characteristic factors and Furstenberg averages", J. Amer. Math. Soc., 20:1 (2007), 53-97.

[35] T. Austin, "On the norm convergence of nonconventional ergodic averages", Ergodic Theory Dynam. Systems, 30:2 (2010), 321-338.

[36] E. Szemerédi, "On sets of integers containing no $k$ elements in arithmetic progression", Acta Arith., 27 (1975), 199-345.

[37] E. Szemerédi, "Regular partitions of graphs", Problèmes combinatoires et théorie des graphes (Orsay, 1976), Colloq. Internat. CNRS, 260, CNRS, Paris, 1978, 399-401. 
[38] H. Furstenberg, "Recurrence in ergodic theory and combinatorial number theory", M. B. Porter Lectures, Princeton Univ. Press, Princeton, NJ, 1981.

[39] H. Furstenberg, "Ergodic behavior of diagonal measures and a theorem of Szemerédi on arithmetic progressions", J. Anal. Math., 31:1 (1977), 204-256.

[40] J. Bourgain, "On triples in arithmetic progression", Geom. Funct. Anal., 9:5 (1999), 968-984.

[41] J. Bourgain, "Roth's theorem on progressions revisited", J. Anal. Math., 104 (2008), 155-192.

[42] B. Green, T. Tao, "The primes contain arbitrarily long arithmetic progressions", Ann. of Math. (2), 167:2 (2008), 481-547.

[43] B. Green, "On arithmetic structures in dense sets of integers", Duke Math. J., 144:2 (2002), 215-238.

[44] И. Д. Шкредов, "Об одной задаче Гауэрса", Изв. РАН. Сер. матем., 200:2 (2006), 176-217; англ. пер.: I. D. Shkredov, "On a problem of Gowers", Izv. Math., 70:2 (2006), 385-425.

[45] I. D. Shkredov, "On a generalization of Szemerédi's theorem", Proc. London Math. Soc. (3), 93:3 (2006), 723-760.

[46] B. Green, T. Tao, "New bounds for Szemerédi's theorem. I. Progressions of length 4 in finite field geometries", Proc. London Math. Soc. (3), 98:2 (2009), 365-392; arXiv: math/0509560.

[47] S. V. Konyagin, I. E. Shparlinski, Character sums with exponential functions and their applications, Cambridge Tracts in Math., 136, Cambridge Univ. Press, Cambridge, 1999, ISBN: 0-521-64263-9, $163 \mathrm{pp.}$

[48] S. V. Konyagin, "Estimates for trigonometric sums and for Gaussian sums", IV Международная конференция "Современные проблемы теории чисел и ее приложения". Ч. ІІІ (Тула, 2001), МГУ, 2002, 86-114.

[49] J. Bourgain, "More on the sum-product phenomenon in prime fields and its applications", Int. J. Number Theory, 1:1 (2005), 1-32.

[50] N. Hegyváry, F. Hennecart, "Explicit constructions of extractors and expanders", Acta Arith., 140:3 (2009), 233-249.

[51] M. Z. Garaev, "The sum-product estimate for large subsets of prime fields", Proc. Amer. Math. Soc., 136:8 (2008), 2735-2739.

[52] D. Hart, A. Iosevich, J. Solymosi, "Sums and products in finite fields via Kloosterman sums", Int. Math. Res. Not. (IMRN), 2007, № 5, rnm007, 14 pp.

[53] I. Shparlinski, "On the solvability of bilinear equations in finite fields", Glasg. Math. J., 50:3 (2008), 523-529; arXiv: 0708.2130v2.

[54] A. Sárközy, "On sums and products of residues modulo p", Acta Arith., 118 (2005), 403-409.

[55] D. Hart, A. Iosevich, "Sums and products in finite fields: an integral geometric viewpoint", Radon transforms, geometry, and wavelets, Contemp. Math., 464, Amer. Math. Soc., Providence, RI, 2008, 129-135; arXiv: 0705.4256.

[56] M.Z. Garaev, V. Garcia, "The equation $x_{1} x_{2}=x_{3} x_{4}+\lambda$ in fields of prime order and applications", J. Number Theory, 128:9 (2008), 2520-2537.

[57] B. Green, "A Szemerédi-type regularity lemma in abelian groups, with applications", Geom. Funct. Anal., 15:2 (2005), 340-376.

[58] B. Green, "Montréal lecture notes on quadratic Fourier analysis", Additive combinatorics, CRM Proc. Lecture Notes, 43, Amer. Math. Soc., Providence, RI, 2007, 69-102; arXiv: math/0604089.

[59] I. Schur, "Über die Kongruenz $x^{m}+y^{m} \equiv z^{m}(\bmod p)$ ", Deutsch. Math. Ver., 25 (1916), 114-117.

[60] W. T. Gowers, "Rough structure and classification", Geom. Funct. Anal., Special Volume - GAFA'2000 (Tel Aviv, 1999), Part I, 2000, 79-117. 
[61] M.-Ch. Chang, "A polynomial bound in Freiman's theorem", Duke Math. J., 113:3 (2002), 399-419.

[62] B. Green, "Arithmetic progressions in sumsets", Geom. Funct. Anal., 12:3 (2002), $584-597$.

[63] B. Green, I. Ruzsa, "An analog of Freiman's theorem in an arbitrary abelian group", J. London Math. Soc. (в печати).

[64] T. Sanders, An application of a local version of Chang's theorem, arXiv: math.CA/0607668.

[65] T. Sanders, "Appendix to «Roth's theorem on progressions», revisited by J. Bourgain", J. Anal. Math., 104 (2008), 193-206; arXiv: 0710.0642v2.

[66] И. Д. Шкредов, "О множествах больших тригонометрических сумм”, Изв. РАН. Сер. матем., 72:1 (2008), 161-182; англ. пер.: I. D. Shkredov, "On sets of large trigonometric sums", Izv. Math., 72:1 (2008), 149-168.

[67] J. Bourgain, "On arithmetic progressions in sums of sets of integers", A tribute to Paul Erdös, Cambridge Univ. Press, Cambridge, 1990, 105-109.

[68] N. H. Katz, T. Tao, "Recent progress on the Kakeya conjecture", Proceedings of the 6th International Conference on Harmonic Analysis and Partial Differential Equations (El Escorial, 2000), Publ. Mat., 2002, 161-179.

[69] T. Tao, "From rotating needles to stability of waves: emerging connections between combinatorics, analysis, and PDE", Notices Amer. Math. Soc., 48:3 (2001), 294-303.

[70] G. Mockenhaupt, T. Tao, "Restriction and Kakeya phenomena in finite fields", Duke Math. J., 121:1 (2004), 35-74.

[71] B. Green, T. Tao, The quantitative behaviour of polynomial orbits on nilmanifolds, arXiv: 0709.3562v1.

[72] B. Green, T. Tao, The equivalence between inverse sumset theorems and inverse conjectures for the $U^{3}$-norm, arXiv: 0906.3100v1.

[73] B. Green, T. Tao, "Quadratic uniformity for the Möbius function", Ann. Inst. Fourier (Grenoble), 58:6 (2008), 1863-1935; arXiv: math/0606087.

[74] T. Tao, T. Ziegler, The inverse conjecture for the Gowers norm over finite fields via the correspondence principle, arXiv: 0810.5527.

[75] A. Samorodnitsky, L. Trevisan, "Gowers uniformity, influence of variables, and PCPs", SIAM J. Comput., 39:1 (2009), 323-360; arXiv:math/0510264.

[76] S. Lovett, R. Meshulam, A. Samorodnitsky, "Inverse conjecture for the Gowers norm is false", STOC'08. Proceedings of the 40th Annual ACM Symposium on Theory of Computing (Victoria, Canada, 2008), 2008, 547-556; arXiv: 0711.3388v1.

[77] W. Rudin, Fourier analysis on groups, Wiley, New York, 1990, (Reprint of the 1962 original).

[78] B. L. Van der Waerden, "Beweis einer Baudetschen Vermutung", Nieuw Arch. Wisk., 15 (1927), 212-216.

[79] P. Erdös, P. Turán, "On some sequences of integers", J. London Math. Soc., 11 (1936), 261-264.

[80] K. F. Roth, "On certain sets of integers", J. London Math. Soc., 28:1 (1953), 104-109.

[81] E. Szemerédi, "Integer sets containing no arithmetic progressions", Acta Math. Hungar., 56:1-2 (1990), 155-158.

[82] D. R. Heath-Brown, "Integer sets containing no arithmetic progressions", J. London Math. Soc. (2), 35:3 (1987), 385-394.

[83] J. Bourgain, "A Szemerédi type theorem for sets of positive density in $\mathbb{R}^{k}$ ", Israel J. Math., 54:3 (1986), 307-316.

[84] E. Szemerédi, "On sets of integers containing no four elements in arithmetic progression", Acta Math. Acad. Sci. Hungar., 20:1-2 (1969), 89-104. 
[85] Y. Kohayakawa, "Szemerédi's regularity lemma for sparse graphs", Foundations of computational mathematics (Rio de Janeiro, 1997), Springer, Berlin, 1997, 216-230.

[86] H. Furstenberg, Y. Katznelson, "An ergodic Szemerédi theorem for commuting transformations", J. Anal. Math., 34:1 (1978), 275-291.

[87] H. Furstenberg, B. Weiss, "A mean ergodic theorem for $N^{-1} \sum_{n=1}^{N} f\left(T^{n} x\right) g\left(T^{n^{2}} x\right)$ ", Convergence in ergodic theory and probability (Columbus, OH, 1993), Ohio State Univ. Math. Res. Inst. Publ., 5, de Gruyter, Berlin, 1996, 193-227.

[88] H. Furstenberg, D. Ornstein, Y. Katznelson, "The ergodic theoretical proof of Szemerédi's theorem", Bull. Amer. Math. Soc. (N.S.), 7:3 (1982), 527-552.

[89] F.A. Behrend, "On sets of integers which contain no three terms in arithmetical progression", Proc. Natl. Acad. Sci. USA, 32 (1946), 331-332.

[90] R. Salem, D. C. Spencer, "On sets which do not contain a given number of terms in arithmetical progression", Nieuw Arch. Wisk. (2), 23 (1950), 133-143.

[91] B. Elkin, An improved construction of progression-free sets, arXiv: 0801.4310v1.

[92] R. A. Rankin, "Sets of integers containing not more than a given number of terms in arithmetical progression", Proc. Roy. Soc. Edinburgh Sect. A, 65 (1961), 332-344.

[93] P. Frankl, G. L. Graham, V. Rödl, "On subsets of abelian groups with no 3-term arithmetic progression", J. Combin. Theory Ser. A, 45:1 (1987), 157-161.

[94] R. Meshulam, "On subsets of finite abelian groups with no 3-term arithmetic progressions", J. Combin. Theory Ser. A, 71:1 (1995), 168-172.

[95] T. Sanders, "Roth's theorem in $\mathbb{Z}_{4}^{n "}$, Anal. PDE, 2:2 (2009), 211-234; arXiv: $0807.5101 \mathrm{v} 1$.

[96] B. Green, Notes on the polynomial Frieman-Ruzsa conjecture, Preprint, 2005.

[97] Н. Г. Чудаков, "О плотности совокупности четных чисел, непредставимых как сумма двух нечетных простых", Изв. АН СССР. Сер. матем., 2:1 (1938), 25-40.

[98] J. G. van der Corput, "Über Summen von Primzahlen und Primzahlquadraten", Math. Ann., 116:1 (1939), 1-50.

[99] S. Chowla, "There exists an infinity of 3-combinations of primes in A.P.", Proc. Lahore Philos. Soc., 6:2 (1944), 15-16.

[100] B. Green, "Roth's theorem in the primes", Ann. Math. (2), 161:3 (2005), 1609-1636.

[101] R. L. Graham, B. L. Rothschild, J. H. Spencer, Ramsey theory, Wiley-Intersci. Ser. Discrete Math. Optim., 2d ed., Wiley, New York, 1990, ISBN: 0-471-50046-1, 196 pp.

[102] B. M. Landman, A. Robertson, "Ramsey theory on the integers", Stud. Math. Libr., 24, Providence, RI, Amer. Math. Soc., 2004, 317, ISBN: 0-8218-3199-2.

[103] Handbook of combinatorics, v. 1, 2, eds. R. L. Graham, M. Grötschel, L. Lovás, Elsevier, Amsterdam; MIT Press, Cambridge, MA, 1995, ISBN: 0-444-88002-X.

[104] R. Rado, "Verallgemeinerung eines Satzes von van der Waerden mit Anwendungen auf ein problem der Zahlentheorie", Sitzungsber. Preuß. Akad. Wiss., Phys.-Math. Kl., 17 (1933), 16-17.

[105] R. Rado, "Some recent results in combinatorial analysis", C. R. Congr. Internat. Math., 2 (1936), 20-21.

[106] W. Deuber, "Partitions theorems for Abelian groups", J. Combin. Theory Ser. A, 19:1 (1975), 95-108.

[107] Р. Грэхем, Начала теории Рамсе, Мир, М., 1984, 98 с.; пер. с англ.: R. K. Graham, Rudiments of Ramsey theory, CBMS Reg. Conf. Ser. Math., 45, Amer. Math. Soc., Providence, RI, 1981, ISBN: 0-8218-1696-9, 65 pp.

[108] P. Frankl, R. L. Graham, V. Rödl, "Quantitative theorems for regular systems of equations", J. Combin. Theory Ser. A, 47:2 (1988), 246-261.

[109] N. Hindman, I. Leader, D. Strauss, "Open problems in partition regularity", Combin. Probab. Comput., 12 (2003), 571-583. 
[110] M. Beiglböck, V. Bergelson, N. Hindman, D. Strauss, "Multiplicative structures in additively large sets", J. Combin. Theory Ser. A, 113:7 (2006), 1219-1242.

[111] V. Bergelson, N. Hindman, I. Leader, "Additive and multiplicative Ramsey theory in the reals and the rationals", J. Combin. Theory Ser. A, 85:1 (1999), 41-68.

[112] H. Lefmann, "On partition regular systems of equations", J. Combin. Theory Ser. A, 58:1 (1991), 35-53.

[113] N. Hindman, D. Strauss, "Algebra in the Stone-ČCech compactification", Theory and applications, de Gruyter Exp. Math., 27, de Gruyter, Berlin, 1998, ISBN: 3-11-015420-X, 485 pp.

[114] V. Bergelson, "Ergodic Ramsey theory - an update", Ergodic theory of $\mathbb{Z}^{d}$-actions (Warwick, 1993-1994), London Math. Soc. Lecture Note Ser., 228, Cambridge Univ. Press, Cambridge, 1996, 1-61.

[115] T. Sanders, Analytic approaches to Schur's theorem, Preprint.

[116] I. D. Shkredov, On monochromatic solutions of some nonlinear equations in $\mathbb{Z} / p \mathbb{Z}$, arXiv: 0909.3269v1.

[117] I. Z. Ruzsa, "Generalized arithmetical progressions and sumsets", Acta Math. Hungar., 65:4 (1994), 379-388.

[118] I. Z. Ruzsa, "An analog of Freiman's theorem in groups", Astérisque, 258 (1999), 323-326.

[119] Yu. Bilu, "Structure of sets with small sumset", Astérisque, 258 (1999), 77-108.

[120] Г. А. Фрейман, Начала структурной теории сложения множеств, Казан. гос. пед. инст., Казань, 1966; англ. пер.: G. A. Freiman, Foundations of a structural theory of set addition, Transl. Math. Monogr., 37, Amer. Math. Soc., Providence, RI, 1973.

[121] I. D. Shkredov, "On sumsets of dissociated sets", Online J. Anal. Comb., 4 (2009), Art. 4, 26 pp.

[122] Ф. Л. Назаров, "Ударное решение задачи о коэффициентах", Алгебра и анализ, 9:2 (1997), 272-287; англ. пер.: F. L. Nazarov, "The Bang solution of the coefficient problem", St. Petersburg Math. J., 9:2 (1998), 407-419.

[123] K. Ball, "Convex geometry and functional analysis", Handbook of the geometry of Banach spaces, v. 1, Elsevier, Amsterdam, 2001, 161-194.

[124] B. Green, "Spectral structure of sets of integers", Fourier analysis and convexity, Appl. Numer. Harmon. Anal., Birkhäuser, Boston, MA, 2004, 83-96.

[125] W. Rudin, "Trigonometric series with gaps", J. Math. Mech., 9 (1960), 203-227.

[126] B. Green, Structure theory of set addition, ICMS Instructional Conference in Combinatorial Aspects of Mathematical Analysis, Edinburgh, 2002, http:// www.dpmms.cam.ac.uk/ bjg23/papers/icmsnotes.pdf.

[127] B. Green, "Some constructions in the inverse spectral theory of cyclic groups", Combin. Probab. Comput., 12:2 (2003), 127-138.

[128] И. Д. Шкредов, "Некоторые примеры множеств больших тригонометрических сумм", Матем. сб., 198:12 (2007), 105-140; англ. пер.: I. D. Shkredov, "Examples of sets with large trigonometric sums", 198:12 (2007), 1805-1838.

[129] А. А. Юдин, "О мере больших значений тригонометрических сумм”, Теория чиceл, ред. Г. А. Фрейман, А. М. Рубинов, Е. В. Новоселов, Калининский гос. ун-т, Калинин, 1973, 163-174.

[130] S. V. Konyagin, V.F. Lev, "On the distribution of exponential sums", Integers, 0 (2000), A1.

[131] V.F. Lev, "Linear equations over $\mathbb{F}_{p}$ and moments of exponential sums", Duke Math. J., 107 (2001), 239-263.

[132] G. Myerson, "How small can a sum of roots of unity be?", Amer. Math. Monthly, 93:6 (1986), 457-459. 
[133] I. Ruzsa, "Arithmetic progressions in sumsets", Acta Arith., 60:2 (1991), 191-202.

[134] G. A. Freiman, H. Halberstam, I. Z. Ruzsa, "Integer sum sets containing long arithmetic progressions", J. London Math. Soc. (2), 46:2 (1992), 193-201.

[135] V.F. Lev, "Blocks and progressions in subset sums sets", Acta Arith., 106:2 (2003), $123-142$.

[136] E. Szemerédi, V. Vu, "Long arithmetic progressions in sum-sets and the number of x-sum-free sets", Proc. London Math. Soc. (3), 90:2 (2005), 273-296.

[137] E. Szemerédi, V. Vu, "Finite and infinite arithmetic progressions in sumsets", Ann. Math. (2), 163:1 (2006), 1-35.

[138] J. Solymosi, "Arithmetic progressions in sets with small sumsets", Combin. Probab. Comput., 15:4 (2006), 597-603.

[139] T. Sanders, "Additive structures in sumsets", Math. Proc. Cambridge Philos. Soc., 144:2 (2008), 289-316; arXiv: math/0605520.

[140] T. Sanders, "A note on Freiman's theorem in vector spaces", Combin. Probab. Comput., 17:2 (2008), 297-305.

[141] N. Alon, J. Spencer, The probabilistic method, Wiley-Intersci. Ser. Discrete Math. Optim., Wiley, New York, 1992, ISBN: 0-471-53588-5, 254 pp.

[142] E. Croot, I. Ruzsa, T. Schoen, "Arithmetic progressions in sparse sumsets", Combinatorial number theory, de Gruyter, Berlin, 2007.

[143] K. T. Smith, "The uncertainty principle on groups", SIAM J. Appl. Math., 50:3 (1990), 876-882.

[144] T. Tao, "An uncertainty principle for cyclic groups of prime order", Math. Res. Lett., 12:1 (2005), 121-127.

[145] S. Guo, Zh.-W. Sun, "A variant of Tao's method with application to restricted sumsets", J. Number Theory, 129:2 (2009), 434-438; arXiv: 0808.0243v1.

[146] P. Stevenhagen, H. W. Lenstra, "Chebotarëv and his density theorem", Math. Intelligencer, 18:2 (1996), 26-37.

[147] Н. М. Коробов, Тригонометрические суммы и их приложения, Наука, М., 1989, ISBN: 5-02-013940-8, 240 с.; англ. пер.: N. M. Korobov, Exponential sums and their applications, Math. Appl. (Soviet Ser.), 80, Kluwer, Dordrecht, 1992, ISBN: 0-7923-1647-9, 209 pp.

[148] И. Е. Шпарлинский, "Об оценках сумм Гаусса", Матем. заметки, 50:1 (1991), 122-130; англ. пер.: I. E. Shparlinskii, "Estimates of Gaussian sums", Math. Notes, 50:1 (1991), 740-746.

[149] D. R. Heath-Brown, S. V. Konyagin, "New bounds for Gauss sums derived from $k$ th powers, and for Heilbronn's exponential sum", Q. J. Math., 51:2 (2000), 221-235.

[150] L. J. Mordell, "On a sum analogous to a Gauss' sum", Quart. J. Math., 3 (1932), 161-167.

[151] T. Cochrane, C. Pinner, "An improved Mordell type bound for exponential sums", Proc. Amer. Math. Soc., 133:2 (2004), 313-320.

[152] E. Bombieri, J. Bourgain, S. V. Konyagin, "Roots of polynomials in subgroups of $\mathbb{F}_{p}^{*}$ and applications to congruences", Int. Math. Res. Not. IMRN, 2009:5 (2009), 802-834.

[153] И. М. Виноградов, Основы теории чисел, 7, Наука, М., 1965, 172 с.; англ. пер. 5-го изд.: I. M. Vinogradov, Elements of number theory, Dover, New York, viii, $227 \mathrm{pp}$.

[154] S. V. Konyagin, "Good distribution of values of sparse polynomials modulo a prime", Analytic number theory, Cambridge Univ. Press, Cambridge, 2009, 289-296.

[155] B. Green, Sum-product phenomena in $\mathbf{F}_{p}$ : a brief introduction, arXiv: 0904.2075v1.

[156] P. Erdös, E. Szemerédi, "On sums and products of integers", Studies in pure mathematics, Birkhäuser, Basel, 1983, 213-218. 
[157] K. Ford, "Sums and products from a finite set of real numbers", Ramanujan J., 2:1-2 (1998), 59-66.

[158] M. B. Nathanson, "On sums and products of integers", Proc. Amer. Math. Soc., 125:1 (1997), 9-16.

[159] G. Elekes, "On the number of sums and products", Acta Arith., 81:4 (1997), 365-367.

[160] B. Barak, R. Impagliazzo, A. Wigderson, "Extracting randomness using few independent sources", SIAM J. Comput., 36:4 (2006), 1095-1118.

[161] M.-Ch. Chang, "On a question of Davenport and Lewis and new character sum bounds in finite fields", Duke Math. J., 145:3 (2008), 409-442.

[162] M. Z. Garaev, "An explicit sum-product estimate in $\mathbb{F}_{p}$ ", Int. Math. Res. Not. IMRN, 2007:11 (2007), rnm035, 11 pp.; arXiv: math/0702780v1.

\section{И. Д. Шкредов (I. D. Shkredov)}

Московский государственный университет

им. М. В. Ломоносова

E-mail: ishkredov@rambler.ru
Поступила в редакцию

07.10 .2009 